\title{
Uncovering Morphoproteomic Relationships Using Probabilistic Graphical Models and Resource Description Framework Knowledgebases
}

\author{
A Dissertation \\ presented to \\ The Faculty of the Graduate School \\ at the University of Missouri-Columbia \\ In Partial Fulfillment \\ of the Requirements for the Degree \\ Doctor of Philosophy \\ By \\ DMITRIY SHIN \\ Dr. Chi-Ren Shyu, Dissertation Supervisor
}

JULY 2012 
(a) Copyright by Dmitriy Shin 2012

All Rights Reserved 
The undersigned, appointed by the Dean of the Graduate School, have examined the dissertation entitled:

\section{Uncovering Morphoproteomic Relationships Using Probabilistic Graphical Models and Resource Description Framework Knowledgebases}

presented by Dmitriy Shin

a candidate for the degree of Doctor of Philosophy

and hereby certify, in their opinions, it is worthy of acceptance.

Dr. Chi-Ren Shyu (advisor)

Dr. Dmitry Korkin

Dr. Mihail Popescu

Dr. Gerald Arthur 


\section{DEDICATION}

I would like to thank my wife, Olga, and my two wonderful sons, Alex and Max, for the support and love that they have given me. I would also like to thank my parents for the happy childhood and the values that they passed to me. 


\section{ACKNOWLEDGEMENTS}

There are many people that helped make this work possible. First of all, I would like to thank my advisor Dr. Chi-Ren Shyu for inspiring me to embark on a journey of a scientific profession and helping me to make things happen. I am also grateful to Dr. Gerald Arthur, who introduced me to the mysteries of the field that has become a great challenge to my abilities, yet also a source of new inspirations. I thank Dr. Popescu for his valuable advice and practical outlook that kept me on track. I am very thankful to Dr. Korkin for encouraging me to aim high and not to be afraid to tackle big problems. I am in debt to MU Informatics Institute's faculty and my fellow students for their useful advice and healthy criticism. I would also like to thank Dr. Douglas Anthony, Dr. Alberto Diaz-Arias, Dr. Bill Caldwell, Dr. Petrides, Bud Smith and many others from the department of Pathology and Anatomical Sciences. I will always remember their support, which helped me to finish this work. 


\section{TABLE OF CONTENTS}

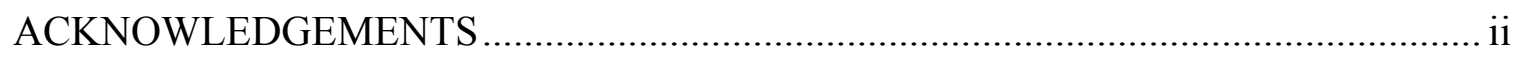

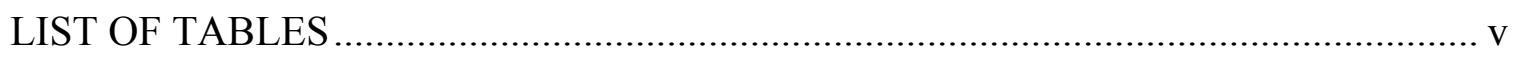

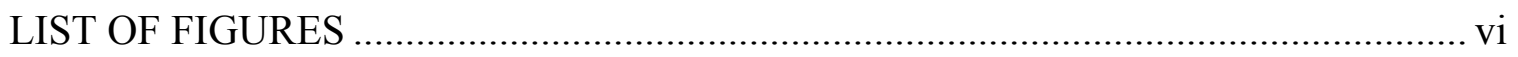

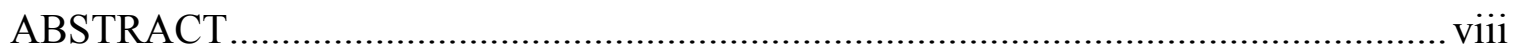

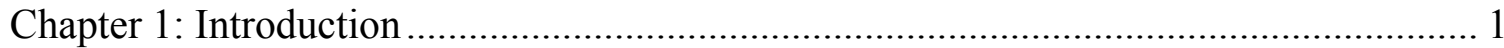

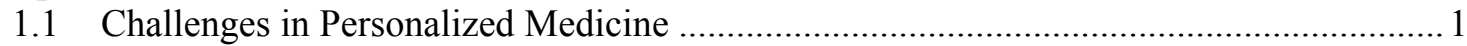

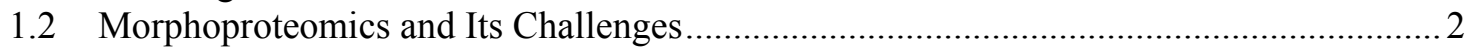

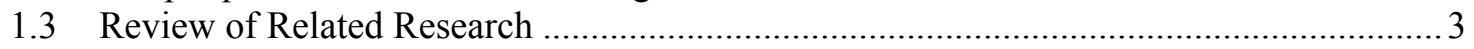

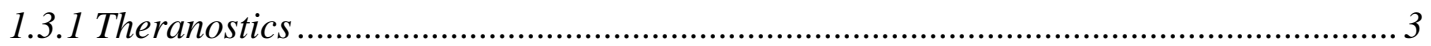

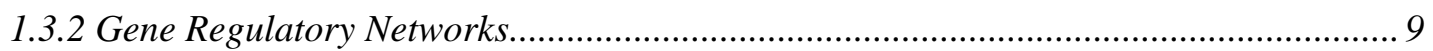

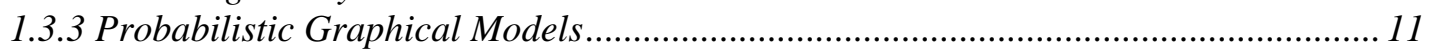

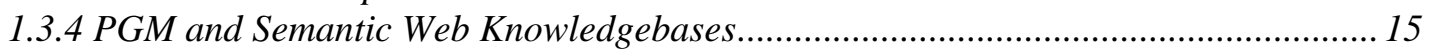

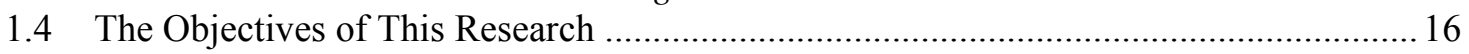

Chapter 2: A Pathologist-In-The-Loop Antibody Test Selection Using Entropy Based

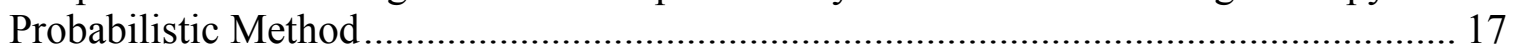

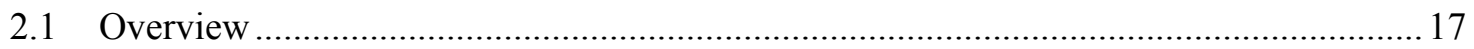

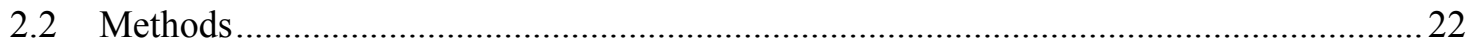

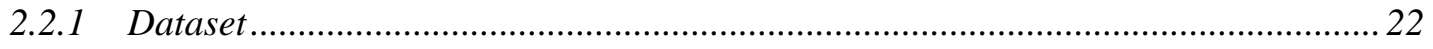

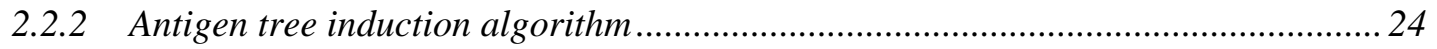

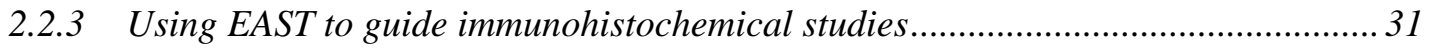

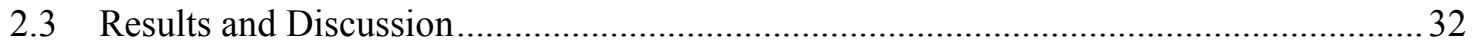

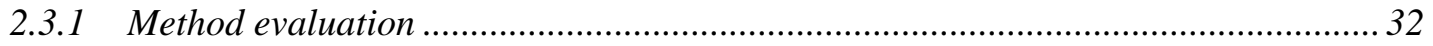

2.3.2 Comparison of the conventional model (WHO, experts' guidelines) and entropy

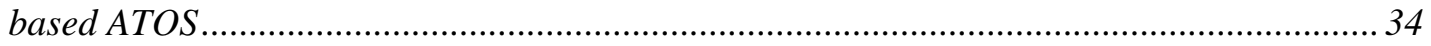

2.3.3 Comparison of manual antibody test selection using ATOS GUI and entropy based

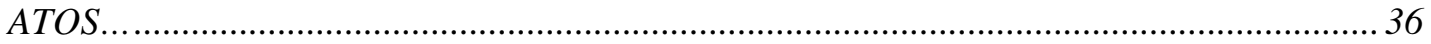

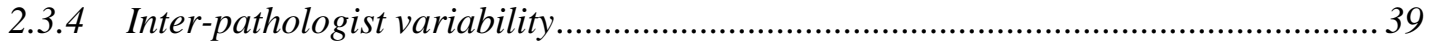

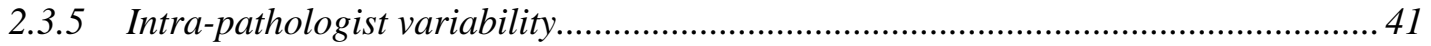

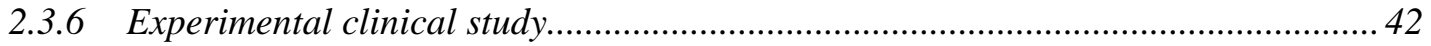

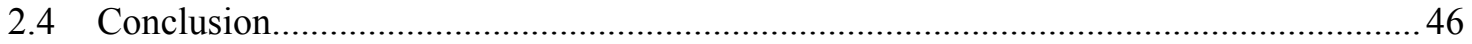

Chapter 3: Uncovering Morphoproteomic Relationships Using Probabilistic Graphical

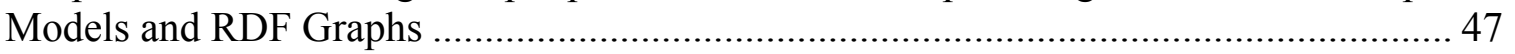

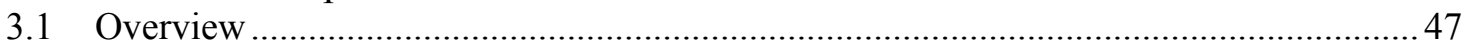

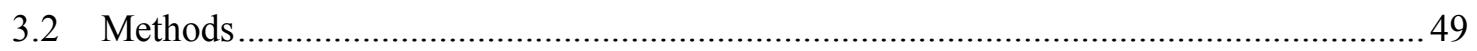

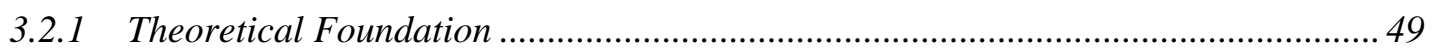

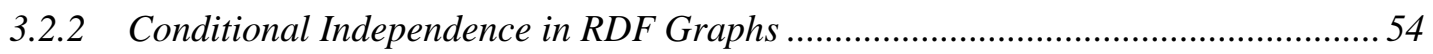

3.2.3 Semantic “Mashup” Knowledgebase of Genetic, Proteomic and Drug Data........... 66

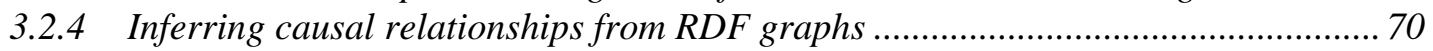

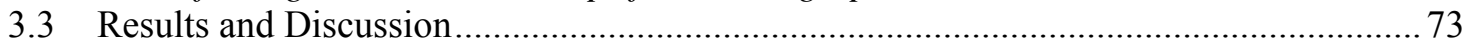

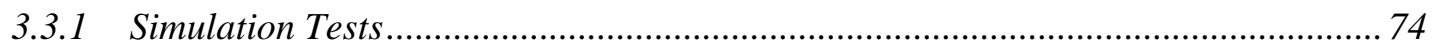

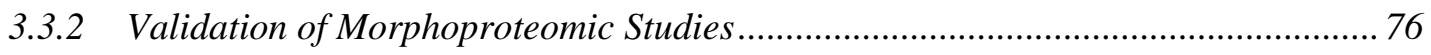

3.3.3 New Findings in Morphoproteomic Studies............................................................. 79 
3.3.4 Discussion of Proposed Approach and Implications to Biomedical Domain ............ 85

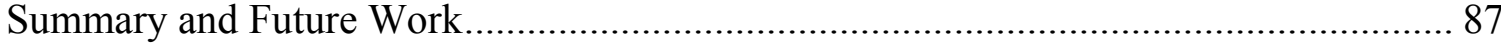

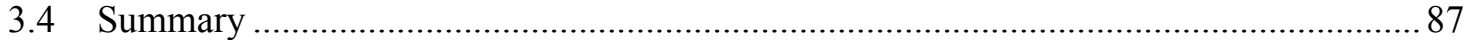

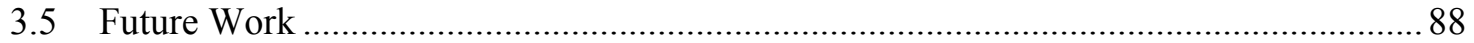

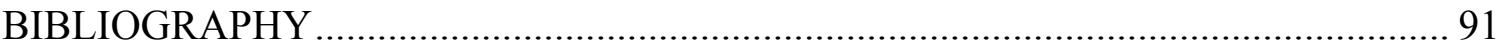

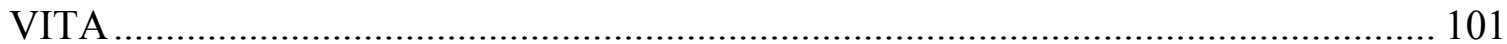




\section{LIST OF TABLES}

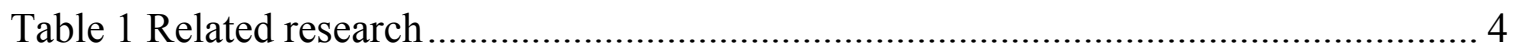

Table 2 Excerpt from a matrix of antigen expression scores across multiple diseases .... 23

Table 3 An example MAEP: “+” expressed, “_” not expressed ...................................... 25

Table 4 Comparison of immunotypic findings for 14 representative lymphomas/leukemias between human expert model (Conv.) and the entropy-based computer algorithm (ATOS) (“+”: positive, “_“: negative outcomes)...................... 35

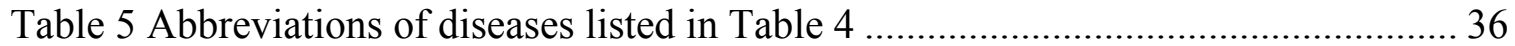




\section{LIST OF FIGURES}

Figure 1 A typical pathology diagnostic workflow …………...................................... 18

Figure 2 Pseudo code of ATOS's EntropySplit() procedure ............................................ 27

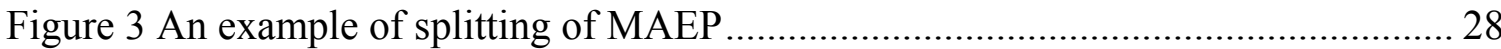

Figure 4 An efficient antigen selection tree (EAS-Tree) based on MAEP from Fig. 3 .... 30

Figure 5 An example of antigen panel tree. The numbers in square brackets identify antigens. The numbers with signs in parentheses indicate outcomes of the testing for

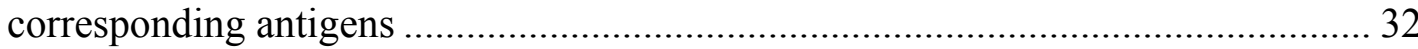

Figure 6 ATOS user interface for diagnostic tree building using automatic entropy-based

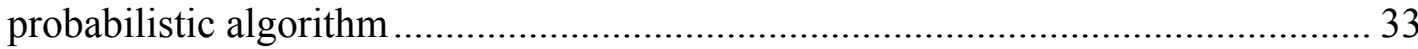

Figure 7 ATOS GUI in simulator program during manual antibody test selection........... 37

Figure 8 Comparison of conventional test selection (WHO, Higgins), manual test selection using ATOS GUI in simulator program and automatic entropy-based

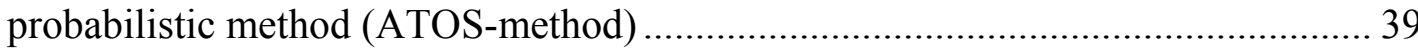

Figure 9 Inter-pathologist variability (two experiments) ................................................... 40

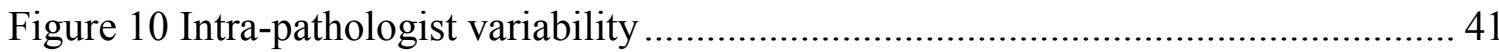

Figure 11 Workflow for inferring casual relationships.................................................... 53

Figure 12 The proposed schema of inferring causal links ................................................ 55

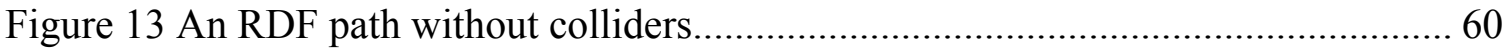

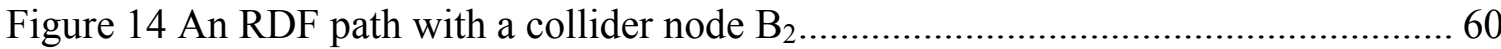

Figure 15 Updated workflow for inferring casual relationships .......................................... 65

Figure 16 Semantic Web "Mashup" Knowledgbase (SWMKB).......................................... 66

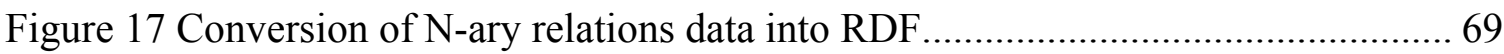

Figure 18 Multiple paths between two RDF nodes ....................................................... 71

Figure 19 Pseudo code of IsIndependent() function ....................................................... 72

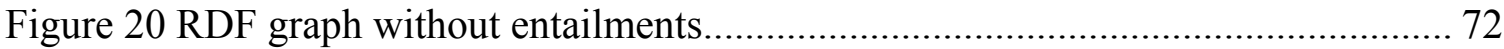

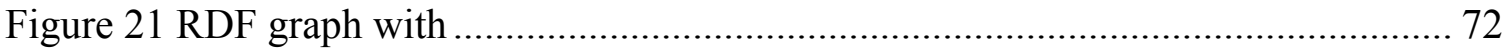

Figure 22 A "synthetic" RDF graph ……………...................................................... 75 
Figure 23 Recovered causal links from "synthetic" RDF graph.................................. 76

Figure 24 Uncovered morphoproteomic relationships Case 1 .................................... 77

Figure 25 Uncovered morphoproteomic relationships Case 2 2................................. 78

Figure 26 Morphoproteomic diagram from Feng et al. ............................................ 81

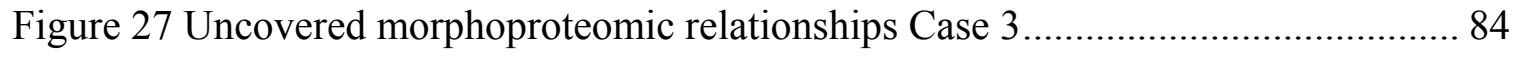




\section{ABSTRACT}

The heterogeneity of individual patient responses to conventional drug therapies is one of the central problems in personalized medicine and has great impact on clinical outcomes. To address this problem a new field of morphoproteomics was recently introduced. Morphoproteomics is a new method aimed at comprehensive analysis of protein circuitries in diseased cells to design effective drug therapies for individual patient cases. However, due to the overwhelming amount of molecular information that needs to be processed, successful adoption of morphoproteomics will greatly depend on availability of a comprehensive computerized knowledgebase and intelligent retrieval technologies.

We have, therefore, initiated new research with the overall goal to develop informatics methods to support morphoproteomic studies. We integrate evidence and information extracted from Whole Slide Imaging (WSI) and Immunohistochemistry (IHC) as well as from a semantic "mashup" of publicly available knowledge sources to provide pathologists a comprehensive picture of morphoproteomic mechanisms. This dissertation introduces novel methods for improving IHC antibody/antigen test selection as well as uncovering morphoproteomic relationships using probabilistic graphical models and Resource Description Framework (RDF) graphs of biomedical knowledgebases. Our methods have great potential to bring a broad impact in to pathology and personalize medicine as well as to be extended to more general systems biology domain. 


\section{Chapter 1: Introduction}

\subsection{Challenges in Personalized Medicine}

Traditionally the standards of medical care focused on studies of large patient populations. Clinical practices based on these standards seldom took into account the variability of individual patients' genetic make-up, which often resulted in significant levels of variability in terms of clinical outcomes. To address this problem a new model of personalized medicine was introduced with the overall goal of redirecting clinical practices toward more individualized care. The early attempts to customize patient care, however, were limited to incorporating family history, environmental conditions and circumstances into the diagnostic and therapeutic decision-making. With the advances in microarray technology, molecular profiling, genetic testing, molecular pathology and related fields, it became possible to define the genetic level of the individual patient's make-up to provide more refined diagnosis and treatment. There was, however, a number of new challenges that personalized medicine brought into the picture [1], with one of the most prominent being the complexity of human cancer and drugs response to it. A pathologist can no longer restrict his work to just identifying a type of malignancy through morphological examination of the tissue. The discoveries of new disease subclasses and phenotypes dictate the need to classify cancer into these more refined categories. Furthermore, pathology seems to be the medical specialty assigned to identify new drug targets, which will help oncologists design efficient therapies tailored to the specifics of individual patient cases. Therefore, pathology as a field of diagnostic medicine has been undergoing a rapid transformation in which molecular pathology is 
gaining ultimate importance; but there are barriers as well. The rapid genome-wide analysis of singular nucleotide polymorphisms (SNPs), which can affect the individual patients responses to drugs, is still a challenge. Moreover, microarray-based expression profiling is not directly indicative of the location of expressed proteins, biomarkers of certain biological processes. For instance, nuclear expression of a protein can constitute its phosphorylation and activation of a signal transduction pathway involved in a tumorigenesis, whereas cytoplasmic expression of the same protein may not be significant. Such cellular translocation events can be detected by microscopic analysis of protein expression patterns on histopathological slides "highlighted" by immunohistochemistry (IHC). One of the first attempts to use this technique to uncover biochemical mechanisms of the individual patient cases and to find new unconventional drug targets was performed by Robert Brown, MD et al. [2, 3]. He and his collaborators used comprehensive assessment of the expression of antibody-linked proteins on histopathological slides to come up with hypotheses on why traditional therapies failed for some patients. They then proposed new drug targets and reported improved clinical outcomes $[4,5]$. The new method called morphoproteomics.

\subsection{Morphoproteomics and Its Challenges}

Morphoproteomics is a new method aimed at comprehensive analysis of protein circuitries in diseased cells to design effective drug therapies in a personalized medicine setting [6]. It combines histopathology, immunohistochemistry, proteomics, molecular pathology and protein biochemistry to draw as complete a picture of involved cellular pathways as possible. Morphoproteomics helps to reveal possible inter- and intra- cellular 
translocations of proteins, i.e., their expression in "unusual" cellular compartments and to create an overall schema of biochemical mechanisms in a given case. The generated schema can be used to identify unconventional drug targets and assess susceptibility to inhibitors and cytotoxic agents that can be combined in effective individualized combinatorial therapies. There have been a number of studies that demonstrated clinical utility of morphoproteomics [7-22]. The problem, however, lies in the fact that morphoproteomics analysis requires accessing a large number of biochemical mechanisms, something that many pathologists may find challenging. As previously demonstrated, the complexity of protein expression patterns creates a combinatorial explosion of data for pathologists to process $[23,24]$. It is even more challenging to identify relevant biological pathways that support certain protein expression patterns. In this dissertation we introduce a computerized knowledge base and retrieval methods to help pathologists extract relevant morphoproteomic information based on IHC protein expression patterns that would have great impact on their abilities to construct morphoproteomic schemas. This might be crucial in finding new drug targets for individual patient cases and therefore reduce the number of "genetic gaps" in personalized medicine setting.

\subsection{Review of Related Research}

\subsubsection{Theranostics}

Several research groups focus on the use of IHC to improve pathology diagnostics and therapeutics, also known as theranostics [25]. There are also research groups that work on data integration problem and use Semantic Web technologies [26] to consolidate 
online knowledge sources into semantic "mashup" that can be queried to produce useful knowledge for biomedical domain. These methods are summarized in Table 1.

Table 1 Related research

\begin{tabular}{|c|c|c|c|c|c|c|c|}
\hline $\begin{array}{l}\text { Research } \\
\text { group }\end{array}$ & Imaging data & $\begin{array}{l}\text { Genetic, genomic } \\
\text { data }\end{array}$ & $\begin{array}{l}\text { Proteomic } \\
\text { data }\end{array}$ & Pathway data & $\begin{array}{l}\text { Disease } \\
\text { data }\end{array}$ & Ontology & Analysis \\
\hline $\begin{array}{l}\text { Robert } \\
\text { Brown et } \\
\text { al [6] }\end{array}$ & $\begin{array}{l}\text { BFM with } \\
\text { IHC slides, } \\
\text { FISH }\end{array}$ & $\begin{array}{l}\text { literature, expert } \\
\text { knowledge }\end{array}$ & $\begin{array}{l}\text { literature, } \\
\text { expert } \\
\text { knowledge }\end{array}$ & $\begin{array}{l}\text { literature, } \\
\text { expert } \\
\text { knowledge }\end{array}$ & $\begin{array}{l}\text { literature, } \\
\text { expert } \\
\text { knowledge }\end{array}$ & N/A & $\begin{array}{l}\text { Manual, based on } \\
\text { subjective impression } \\
\text { of protein level } \\
\text { expression and } \\
\text { individual researchers' } \\
\text { knowledge }\end{array}$ \\
\hline $\begin{array}{l}\text { Russell } \\
\text { Higgins } \\
\text { et al [27] }\end{array}$ & $\begin{array}{ll}\text { BFM } & \text { IHC } \\
\text { slides } & \end{array}$ & $\begin{array}{l}\text { expert knowledge } \\
\text { literature }\end{array}$ & $\begin{array}{l}\text { expert } \\
\text { knowledge } \\
\text { literature }\end{array}$ & $\begin{array}{l}\text { expert } \\
\text { knowledge } \\
\text { literature }\end{array}$ & $\begin{array}{l}\text { expert } \\
\text { knowledge } \\
\text { literature }\end{array}$ & N/A & $\begin{array}{l}\text { Manual, based on } \\
\text { subjective impression } \\
\text { of protein level } \\
\text { expression rand } \\
\text { individual researchers' } \\
\text { knowledge }\end{array}$ \\
\hline $\begin{array}{l}\text { Michael } \\
\text { Feldman } \\
\text { [28] }\end{array}$ & $\begin{array}{l}\text { BFM IHC with } \\
\text { multi spectral } \\
\text { CRI systems, } \\
\text { Aperio WSI }\end{array}$ & $\begin{array}{l}\text { expert knowledge } \\
\text { literature }\end{array}$ & $\begin{array}{l}\text { expert } \\
\text { knowledge } \\
\text { literature }\end{array}$ & $\begin{array}{l}\text { expert } \\
\text { knowledge } \\
\text { literature }\end{array}$ & $\begin{array}{l}\text { expert } \\
\text { knowledge } \\
\text { literature }\end{array}$ & N/A & $\begin{array}{l}\text { Computer-based image } \\
\text { processing algorithms. } \\
\text { System for de- } \\
\text { multiplexing stains. }\end{array}$ \\
\hline $\begin{array}{ll}\text { Satya } & \text { S. } \\
\text { Sahoo } & \text { et } \\
\text { al [29] } & \end{array}$ & N/A & $\begin{array}{l}\text { Entrez } \text { Gene (EG) } \\
\text { digital knowledge } \\
\text { base }\end{array}$ & N/A & N/A & $\begin{array}{l}\text { NCBI } \\
\text { OMIM - } \\
\text { database of } \\
\text { genetic } \\
\text { disorders }\end{array}$ & $\begin{array}{l}\text { Gene } \\
\text { Ontology } \\
\text { (GO) }\end{array}$ & $\begin{array}{l}\text { Sematic Web based } \\
\text { framework provides } \\
\text { storage of data as } \\
\text { RDF, inference } \\
\text { through OWL and } \\
\text { ontology and querying } \\
\text { through SPARQL }\end{array}$ \\
\hline $\begin{array}{ll}\text { Satya } & \text { S. } \\
\text { Sahoo } & \text { et } \\
\text { al [30] } & \end{array}$ & N/A & $\begin{array}{l}\text { Entrez Gene (EG), } \\
\text { HomoloGene }\end{array}$ & N/A & $\begin{array}{l}\text { KEGG, } \\
\text { Reactome, } \\
\text { BioCyc }\end{array}$ & N/A & $\begin{array}{l}\text { GO, } \\
\text { Biopax }\end{array}$ & $\begin{array}{l}\text { Sematic Web based } \\
\text { framework provides } \\
\text { storage of data as } \\
\text { RDF, inference } \\
\text { through OWL and } \\
\text { ontologies, SPARQL }\end{array}$ \\
\hline $\begin{array}{l}\text { Charles } \\
\text { Vaske et } \\
\text { al [31] }\end{array}$ & N/A & Microarray Data & $\begin{array}{l}\text { Microarray } \\
\text { Data }\end{array}$ & NCI PID & N/A & BioPax & $\begin{array}{l}\text { SPARQL, Factor } \\
\text { Graphs modeling, } \\
\text { multidimensional } \\
\text { modeling using } \\
\text { probabilistic inference }\end{array}$ \\
\hline $\begin{array}{l}\text { The } \\
\text { proposed } \\
\text { research }\end{array}$ & IHC WSI & EG, GEO & $\begin{array}{l}\text { UniProtKB , } \\
\text { HPRD }\end{array}$ & $\begin{array}{l}\text { KEGG, } \\
\text { Reactome, } \\
\text { BioCyc, } \\
\text { BioCarta, } \\
\text { NCI PID }\end{array}$ & OMIM & $\begin{array}{l}\text { GO, } \\
\text { BioPAX, } \\
\text { Uniprot, } \\
\text { custom } \\
\text { ontologies }\end{array}$ & $\begin{array}{l}\text { Semi-automatic } \\
\text { image processing, } \\
\text { Sematic Web, RDF, } \\
\text { OWL, rulebases, } \\
\text { SPARQ, PGM }\end{array}$ \\
\hline
\end{tabular}

BMF - bright field microscopy, IHC - immunohistochemistry, FISH - fluorescence in situ hybridization, CRI - Cambridge Research and Instrumentation Inc, WSI - Whole Slide Imaging, NCBI - National Center for Biotechnology Information, RDF - Resource Description Framework, OWL - Web Ontology Language, SPARQL - Semantic Web query language, CV - computer vision, IM image processing, NCI PID - National Cancer Institute Pathway Interaction database, PGM - Probabilistic Graphical Models, HPRD Human Protein Reference Database, OMIM - NCBI Mendelian Inheritance in Man

Brown and his group at Department of Pathology in University of Texas Health Science Center at Houston pioneered new methods of morphoproteomics and morphogenomics [6] (first research group in Table 1). One of the main goals for these new methods is to guide targeted cancer therapies. By studying the morphology of protein expression (e.g. nuclear vs plasmalemmal vs cytoplasmic expression) from IHC 
slides (morphoproteomics), the researchers try to explain underlying biological processes in a diseased tissue. The assumption behind their method is that a more thorough than "conventional" study of protein expression from IHC and FISH probes can point out what signal transduction pathways are activated, whether the drug worked or not, and finally, based on discovered facts, design effective cancer therapies (e.g. combinatorial therapies integrating cytotoxic and differentiating agents and signal transduction inhibitors). They use expert- and literature-derived knowledge as well as manual assessment of protein expression patterns by bright field microscopy (BFM) and semiquantitative analyses of combined IHC scores to manually analyze biological processes for therapy design. They emphasize the fact that a response to the same therapy can be inconsistent across different patient cases. Because of that, more thorough analyses of companionate cells and processes need to be performed to attempt to resolve such inconsistencies.

Russell Higgins, Jennifer Blankenship and Marsha Kinney at Department of Pathology in University of Texas Health Science Center at San Antonio have published a work with an overall guidance for practicing pathologists for using IHC in diagnosis of Hodgkin and Non-Hodgkin lymphomas [27]. The authors suggest carefully designed panels to study and diagnose malignant hematopoietic tumors. They point out that an explosion in the number of antigens (proteins) in cluster designation system (CD) "necessitates judicious use of carefully selected panels rather than a 'shotgun approach' to diagnosis." The researchers discuss expression patterns of many proteins associated with lymphomas and show how to determine cell lineage and to detect specific malignancies. Higgins et al use IHC to phenotype abnormal population detected by 
conventional morphology on hematoxylin and eosin (H\&E) stained slides, characterize an abnormal population detected by flow cytometry and to screen "suspected reactive" tissue for subtle abnormalities. The authors use conventional BFM with IHC to study protein expression patterns and expert knowledge for manual analysis of the biological processes. Higgins et al. also warned about the difficulties (pitfalls) in the interpretation of IHC data.

Michael Feldman at the University of Pennsylvania Health System and his collaborators at Cambridge Research \& Instrumentation, Inc. (CRi), Rutgers University and Rensselaer Polytechnic Institute have been practicing a new method that they named slide-based histocytometry [28]. They developed a set of staining protocols and a range of computational algorithms to analyze and interpret protein expression patterns for therapeutic and diagnostic purposes. The developed methods include protein quantification algorithms, color de-multiplexing approaches, image segmentation and tumor classification software. The authors base their analysis on conventional, expert and literature-derived knowledge of biological processes in a manual manner.

Sahoo et al. were among the first to show that publicly available electronic biomedical knowledge sources such as Entrez Gene (EG) [32], Gene Ontology (GO) [33] and OMIM [34] databases can be linked in a unified framework based on RDF [29]. This framework can supply not only the information directly stored in the above mentioned sources, but also information about inferred relationships. For instance, to find a relationship between not just a particular gene and a particular disease but also between related genes and related disorders would require writing specialized software or manual effort. This is because these online resources were primarily designed for consultation by 
humans. With EG, OMIM and GO imported into Sematic Web repository based on Oracle $10 \mathrm{~g}$, the authors were able to demonstrate that such queries can be handled automatically. For example, a query: "Find all genes annotated with glycosyltranferaserelated terms in GO and associated with disease congenital muscular dystrophy in OMIM" will return all genes carrying molecular function glycosyltranferase as well as all "descendants" with specialized types of glycosyltransferase, such as acetylglucosaminyltransferase. This is possible due to inference capabilities of Web Ontology Language OWL [35] and query language SPARQL [36]. The research was not concerned with disease diagnosis or treatment. Its primary goal was to explore Sematic Web capabilities for data integration of publicly available biomedical sources to answer complex biomedical questions.

In another work [30] Sahoo et al. added information about cellular pathways. They created the Entrez Knowledge Model (EKoM) genetic semantic "mashup" and then incorporated knowledge from KEGG [37], Reactome [38] and BioCyc [39] pathways databases with the help of standardized Biopax ontology [40]. By using pathway information, researchers had demonstrated that even simple SPARQL queries could easily identify hub genes, i.e., genes that participate in a large number of pathways or interact with many other gene products.

In [31] Vaske et al. used breast cancer and glioblastoma multiform microarray data to infer gene involvement in signal transduction pathways. They modeled it by factor graphs and probabilistic inference using a proposed PARADIGM method. The main goal was to generate a matrix of integrated pathway activity (IPA) for each gene and assess involvement of each gene in a pathway using statistics. The involvement of a gene in a 
pathway is assessed based on a deviation of a gene measurement from a null distribution, which they generate based on "within" permutation. They then ordered pathways by gene involvement and visualized using a novel CircleMaps tool. The authors claim that their factor graph based modeling can incorporate several functional aspects for each gene like DNA methylation, copy number, overexpression etc.

As can be seen from this review of related research, the first two groups are concerned with pathology theranostics issues and employ tissue-based proteomics methods (i.e., IHC). However, they use traditional BFM tools and expert knowledge coupled with the review of the literature to analyze IHC protein expression patterns and make sense out of it. The next group in Table 1 uses advanced WSI and multispectral microscopy imaging (CRi) technologies in addition to BFM. They also develop computer algorithms and design staining protocols for more quantitative analysis of IHC protein expression. But they still use expert knowledge to recreate a picture of biological processes. The Sahoo et al. group, on the other hand, utilizes computer knowledge-based systems to derive biological knowledge. Their frameworks, however, are not linked to any imaging data and are not designed to recreate the morphoproteomic picture of biological processes in a given tissue sample. Vaske et al. used microarray data to infer involvement of a gene in a pathway as well as curated data from NCI pathway database and BioPax ontology. However, data from microarray experiments lack locational information, i.e., it just shows expression (or methylation etc) levels but not where the particular gene product is expressed, which is an important diagnostic factor in pathology. For example, if a certain protein is expressed in the cytoplasm, it may be of no importance; if it is expressed in the nucleus, it may indicate phosphorylation and 
activation of a "corresponding" pathway. Inconsistency of some high-throughput experiments and, therefore, inability to use it in a personalized medicine setting can be attributed to this lack of locational information.

\subsubsection{Gene Regulatory Networks}

Modeling of Gene Regulatory Networks (GRN) using computational means is a related research area that is also concerned with studying relationships among genes and gene products (protein, transcription factors). However, GRN modeling sets more "basic research" goals of discovering or confirming biological networks in which gene and their products interact rather than identifying activated networks and mechanisms based on clinical tests.

Here we briefly list traditional methods for modeling GRN that have been developed over the past decades.

Boolean Networks were first proposed by Kaufman [41] and have been since used to model GRNs [42]. In these networks each node represents a dichotomous variable, the two values of which code for active and inactive states of the underlying biological entity (e.g. gene). The edges of a Boolean Network correspond to Boolean operations AND, OR and NOT. The whole network of $n$ nodes can be viewed as a binary feature vector going through $2^{\text {n }}$-dimensional space of different states. Some equilibrium states can correspond to gene regulatory processes under certain conditions such as disease or immunoreactive response of an organism. The goal of learning Boolean Network is to find a Boolean function for each gene in such a way that it fits the observed data. There are a number of 
algorithms for learning Boolean Network, including REVEAL by Liang et al. [43]. Boolean Networks are limited by the fact that each gene can be represented only by two states, the values of which are approximated by a discretization procedure. That raises a question as to what is the appropriate threshold to process continuous gene expression values. A new type of Probabilistic Boolean Networks (PBN) was proposed by Shmulevich [44] that overcomes the deterministic nature of the traditional Boolean Networks. PBNs model the stochastic nature of gene regulation by introducing the state transition mechanism that is dependent on a random variable. One example of usage of PBN to model GRNs is T lymphocytes receptor signaling in which PBN models immune response to different foreign entities [44].

Differential and Difference equations model dynamic aspects of GRN (i.e., changes in gene expression) [45]. Due to potential existence of multiple solutions for ordinary differential equations (ODE), some constraints are utilized to reduce complexity. Such constraints might be represented by a prior knowledge or other simplifications. It is believed that gene regulatory processes have a complex non-linear character. Therefore, even though "linearization" can make modeling simpler, linear models still are not sufficient to model large scale GRNs from gene expression data accurately [46]. Still, there are both linear and non-linear differential equations that can capture kinetics of GRNs, with the latter having limitations related to the computational difficulties.

Bayesian Networks (BNs), in which the structure of a network is represented by directed acyclic graph (DAG) and nodes are represented by random variables, can be used to capture stochastic nature of gene regulation. The expression values of each gene 
are determined by the Bayes rule and local Markov property [47], which flexibly incorporates prior knowledge in the process of GRN inference to generate a correct network structure. There are several aspects of BNs that we will discuss in more detail in the Section 1.3.3 as well as in Chapter 3 as they represent the main underlying formalism that is used in this dissertation's research methods.

There are a number of other GRN architecture approaches such as Petri Nets, Hybrid Models and Finite State Linear Models (FSLMs) which can all be viewed as extensions of graph models and have been successfully used to model, for example, metabolic networks or to incorporate discrete and continuous aspects of DNA expression. For a review of these models see Schlitt et al. [45].

\subsubsection{Probabilistic Graphical Models}

Probabilistic Graphical Models (PGMs) are a large family of representational and computational methods [47-54]. The common feature of all PGMs is that they all combine probability and graph theories to manage complexity of stochastic processes and provide means for inference. In fact, PGMs can be considered as an underlying fundamental formalism for many methods used in machine learning, statistics, physics and other fields. PGMs manage complexity through a decomposition of a large system into "digestible" parts, whose parameters can be successfully learned. These "parts" together represent one network that can then be used for inference. Depending on the type of decomposition there have been developed several constituent formalisms including Markov Random Fields (MRFs), Bayesian Networks (BNs) and recently 
introduced Hybrid Random Fields (HRFs). Since our main research focus in this dissertation is a development of a methodology to manage complexity of IHC and morphoproteomic studies using BNs, we will briefly describe MRFs and HRFs and then discuss BNs in more detail.

The first usage of MRFs can be traced to the field of stochastic mechanics, to soknown Ising model [55]. MRFs represent a joint probability distribution of a stochastic process by an undirected graph, where each node is a random variable and each maximal clique (a sub-graph where all vertices are connected) is assigned a potential function $\varphi_{c}$. An argument of a potential function $\varphi_{c}$ is a state of a clique $C$ and output is a nonnegative number. So, the overall joint probability distribution is computed as follows:

$$
P(\boldsymbol{x})=\frac{1}{Z} \prod_{\varphi_{c}} \varphi_{c}\left(\boldsymbol{x}_{c}\right) \quad \text { Eq. } 1
$$

where $\boldsymbol{x}_{C}$ is a state of the clique $C$ and $Z$ is a normalization factor, which is the sum over all products of all possible configurations of $\boldsymbol{x}$. After some manipulations it can be shown that this distribution is actually a Gibbs distribution. The connection between Gibbs distribution and topological nature of MRF is better understood by the HammersleyClifford theorem. This theorem states that to say that a process can be modeled by Gibbs distribution is equivalent to saying that the process satisfies the Markov property and vice versa [56]. The Markov property reflects the statistical independence of a node in a network from all other nodes given the outcomes of that node's immediate neighbors. In context of MRFs it is called Markov Blanket and in context of BNs it is called Directed Markov Property. MRFs can be used to model processes where the direction of 
connections among entities is not specified (e.g. correlation). It is relatively difficult to learn the structure of an MRF from experimental data.

Hybrid Random Fields [57] is a new approach that was designed to overcome some weak properties of BNs and MRFs. In particular, learning structure of a BN network can be very costly in a high-dimensional domain. Even though there are many heuristic methods for learning parameters and structures of BNs, these methods may not scale well because the number of possible DAGs grows exponentially with the number of nodes. Since heuristic methods search through the space of all possible DAGs, such methods may quickly become computationally intractable. In HRFs parameter learning is processed by local BNs, consisting of a set of only suitable variables. Therefore, the whole HRF is modeled as a union of such local BNs, each of which can be learned relatively efficiently. Both parameter and structure learning algorithms have been proposed by Freno et al. [56] for discrete distributions. Later, HRFs were extended to continuous variable domains.

BNs can be taken to be, perhaps, the most popular graphical methods to model conditional probability distributions. One of the first investigators to propose a rigorous mathematical formalism for BNs was Judea Pearl [52]. He introduced the notions of $d$ separation, minimal I-Map and faithfulness condition that are considered to be the main fundamental principles underlying all graphical models. In this dissertation Pearl formalism is exploited to derive causal relationships among proteins and signaling pathways (see Chapter 3). Namely, we extend d-separation principles into the domain of RDF graphs and apply it to generate a set of independence statements, which can then be 
fed into structure learning algorithms. There are two main tasks associated with learning BNs: (i) parameter learning and (ii) structure learning.

Parameter learning in $\mathrm{BNs}$ is a task of assigning conditional probabilities to each node in a BN network, given that the BN's structure is provided. A probability is computed for all node values (discrete case) for every possible instantiation of parents' node values. For example in a binary variable $\mathrm{BN}$ network, if a node $x 1$ has two parents $x 2$ and $x 3$, we need to learn eight conditional probabilities: $P(x 1=0 \mid x 2=0, x 3=0)$, $P(x 1=0 \mid x 2=0, x 3=1), P(x 1=0 \mid x 2=1, x 3=0), P(x 1=0 \mid x 2=1, x 3=1), P(x 1=1 \mid x 2=0, x 3=0)$, $P(x 1=1 \mid x 2=0, x 3=1), P(x 1=1 \mid x 2=1, x 3=0)$, and $P(x 1=1 \mid x 2=1, x 3=1)$. These conditional probabilities can be readily approximated using frequencies and computed from experimental data.

Structure learning is a more computationally challenging task because of the large number of possible DAGs that need to be considered. Two distinct approaches have been developed: (i) score-based structure learning and (ii) constrained-based structure learning [58].

In score-based structure learning, some kind of heuristic algorithm (e.g., hillclimbing) is used to iterate over DAG search space. Each DAG is assigned a score. The DAG with the best score is then selected as a solution. There are several types of scores that can be used including Minimum Description Length (MDL) [59] and BDe score [60, 61]. 
Constrained based structure learning of BNs exploits some kind of restrictions to generate a graph of a BN. SGS algorithm named after its authors Spirtes, Glymour and Scheines [53] is perhaps the most simple algorithm that tries to reconstruct a network by removing an edge in between two nodes if they are conditionally independent given sets of other nodes. Since, the number of such sets can be big, SGS algorithm can run very slow. The PC structure-learning algorithm optimizes search by pre-ordering the conditioned sets by their cardinality, from smallest to largest, so the complexity is bounded by the largest degree in the graph of a BN. The GS algorithm exploits sparseness of BN graphs to avoid processing a fully connected graph as is done in SGS and PC algorithms [58].

\subsubsection{PGM and Semantic Web Knowledgebases}

There have been a number of research projects involving probabilistic graphical models and Semantic Web technologies. Perhaps the most obvious way is to use RDF graphs to encode for probabilistic information (see for instance [62]). In this case, RDF plays a role of the "storage" for a conditional probability distribution. Potential benefits may include data integration between heterogeneous systems that need to exchange, for instance, BNs for processing. Since there is not a standard persistent model for BN, this RDF-based method for storing BN can find utilization in design of software systems.

There have also been attempts to derive the structure of BNs from ontologies [63-65]. Since, the main goal in these investigations was to show a possibility of an automatic reconstruction of a BN from ontology, the resulting network represented a graph of 
ontological concepts rather their instances. For example, in [64] the resulting BN shows probabilistic relation among such concepts as "Threat" and "Asset.". In our case, we are most interested to establish relationships not just among proteins and pathways as concepts (or families of concepts) but particular "instances" of proteins such as AKT, NF-kappa-B and others. To our knowledge, there has not been any work done to develop a methodology that uses RDF graphs of instance data knowledgebases to derive structure of a graphical model that reflects relationships among protein and pathways.

\subsection{The Objectives of This Research}

In this work we set two goals: 1) to develop a computerized method to manage complexity of different IHC tests, and 2) to develop a framework that will allow generating morphoproteomic schemas based on a Semantic Web driven knowledge base and probabilistic graphical models. The central part of the system is a semantic "mashup" of a more comprehensive set of biomedical databases than was used by Sahoo et al. (see Table 1). Moreover, the biomedical problem that this research tackles is more challenging than the one from Sahoo's group. We are interested not just in finding direct relationships between stand alone biological entities, but more in generating a comprehensive schema of processes involved in tumorigenesis, given protein expression patterns from a particular patient case. We will use the words antibody, antigen and protein interchangeably in this document. 


\section{Chapter 2: A Pathologist-In-The-Loop Antibody Test Selection Using Entropy Based Probabilistic Method}

This chapter introduces the ATOS - Antigen Tests Optimized Selection method, a novel informatics tool to help pathologists streamline IHC studies.

\subsection{Overview}

An extensive corpus of fundamental medical knowledge has been gained through decades of the scientific examination of tissues stained with the basic hematoxylin and eosin stains. Countless studies have identified precise diagnostic entities based on fine visual and logical distinctions evident from alterations of cellular morphology [66]. Even more precise diagnostic entities are being discovered based on genomic and proteomic signatures of the diseases and their subclasses; hence, it has become increasingly important to utilize methods of diagnostic molecular pathology to provide precise diagnosis as well at the genomic/proteomic level, which can lead to improved treatment and therefore better clinical outcomes. However, there are several challenges associated with genomic/proteomic studies. 


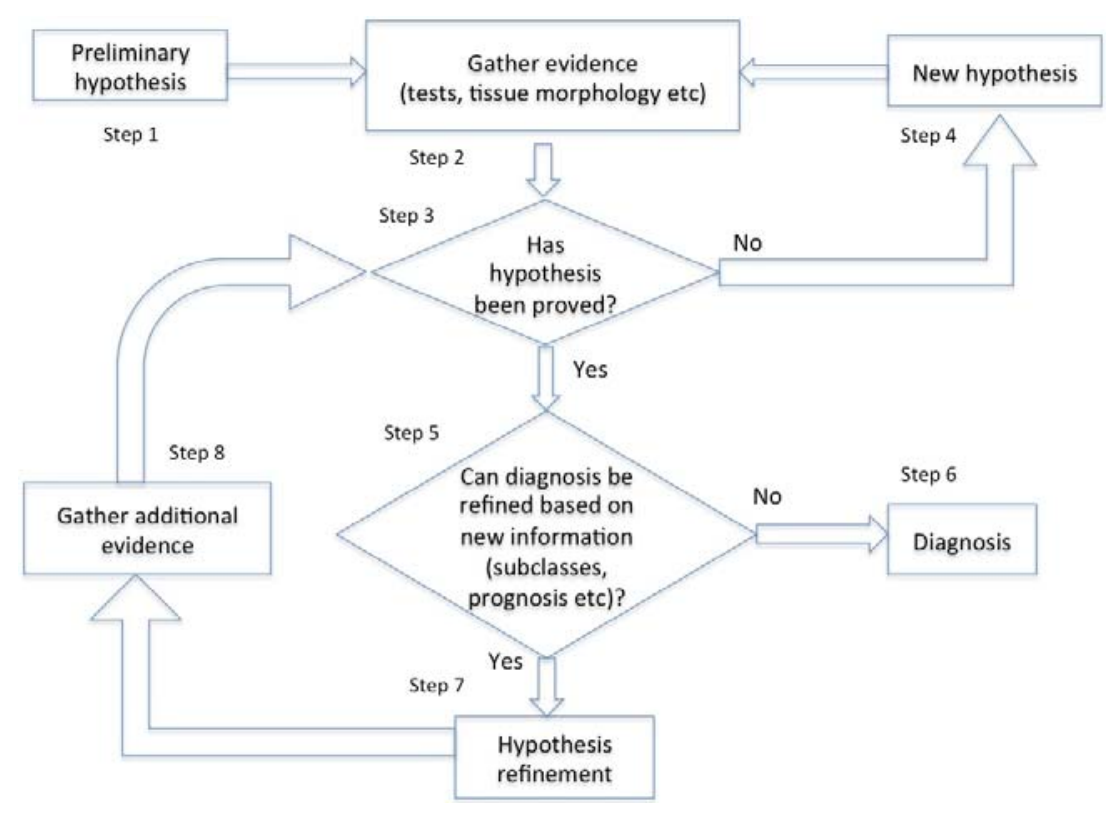

Figure 1 A typical pathology diagnostic workflow

Fig. 1 depicts a typical pathology diagnostic workflow involving extensive iterative processes. A diagnostic process usually starts with some preliminary hypothesis (Step 1) after receiving initial information and gathering initial evidence. If further morphological observations and clinical findings (Step 2) reject that hypothesis (Step 3), a new hypothesis is generated (Step 4). However, if the evidence supports the hypothesis (Step 3), the pathologist may or may not immediately report it as a diagnosis. Instead, he or she might be interested in knowing if the diagnosis can be refined to reveal possible disease subclasses and/or additional important genomic and proteomic characteristics (Step 5). If so, a refined hypothesis is generated (Step 7). New evidence is then collected (Step 8) and analyzed to see if the refined hypothesis can be accepted (Step 3). Steps 3, 5, 7, and 8 can be repeated in a loop several times until after all possible refinement attempts, which can provide precise evidence for treatment, are exhausted. This diagnostic loop is a technique that pathologists use to gradually drill down to the most precise diagnosis. The 
technique can be especially important in solving difficult cases and/or to tailor treatment to individual patient genomic makeup in a personalized medicine setting. Among the most important types of evidence being collected during the diagnosis refinement step are the results of application of antibody-linked stains, a.k.a. immunohistochemistry (IHC). IHC tests have permitted the highly specific identification of a diverse variety of cellular proteins that play an essential role in the molecular pathology of a wide range of diseases. These diagnostic tools have also allowed the emergence of new fields of morphoproteomics and morphogenomics [6], which have great potential to provide more specific and accurate diagnoses and, consequently, a more reliable estimation of prognosis. As a consequence of this transformative technology, great demands have been imposed upon the integrative intellectual skills of even the most experienced and welltrained surgical pathologists. The mental retention and recall of the large number of facts generated during diagnosis refinement in the interpretation of specific protein patterns in biopsies from even a single organ system can be problematic. For example, just for one family of diseases, lymphomas, which can have more than 60 variations [67], there could be more than 80 different antigens [68,69], that may need to be analyzed before an interpretation of the underlying biological processes of a given case can be obtained [27]. Currently, the cluster designation system includes more than 400 antigens [70] and the number of proteins functioning as biomarkers keeps increasing with continuous developments in molecular biology [71-73]. Since pathologists can consider more than just two states of protein expression (e.g. weakly expressed, strongly expressed etc.), the number of all possible combinations of antigen expression creates a combinatorial explosion that a practicing pathologist can face during the diagnostic process. 
Typically, IHC is performed using panels of antibodies ranging from five to a dozen or more as judged appropriate for the tissue and tumor being examined. The composition of a panel is selected to both confirm and rule out diseases for further diagnostic consideration. The exact content of antibody test panels and the sequence in which tests are performed can sometimes vary significantly from pathologist to pathologist (interpathologist variability). Moreover, the same pathologist may order different tests for very similar cases on different days (intra-pathologist variability). Such variability results in high inconsistency rates in pathology disease diagnosis [74]. It is very easy for a pathologist to overlook the inclusion of a certain antigen into the panel and miss an opportunity to obtain a crucial clue about the disease in question. On the other hand, a pathologist might include unnecessary tests in the panels with discriminatory power that may not be required. Therefore, it appears to be inefficient to design panels of fixed size to examine predetermined disease groups rather than to permit a dynamic selection of the antibodies required to evaluate a more realistic set of diseases with which the pathologist may be confronted.

Given the central diagnostic importance of IHC and predicted rapid increase in its utilization, it is timely to question whether there may be more effective and efficient schema for the implementation of IHC than those that have been developed empirically over decades. It is becoming increasingly important for modern healthcare to deliver cost-effective service in general and reduce cost of pathology and laboratory medicine in particular [75]. Hence, a method that can save both in pathologists' time and cost of reagents can find widespread application in the Digital Pathology era [76, 77], when 
pathology diagnosis is more and more reliant on the assistance of information technology and computational tools [28].

Previously, there have been attempts with certain degrees of success to develop computerized methods to assist pathologists in their decision-making. Early studies include Pathfinder expert system created by Heckerman et al. [78]. While this method helps to organize the diagnostic process using etiological and morphological features, it is not suitable for the new challenges and complexity posed by increasing usage of IHC in the pathology workflow. A number of other works had used probabilistic methods to improve decision-making in pathology [79, 80]. More recent studies, using a noncomputational approach, on solving problems due to the complexity of IHC tests include works by Taylor $[68,69]$.

We have identified a few distinct features of the diagnostic workflow discussed earlier (Fig. 1) that, in our view, make it hard to directly apply most of the abovementioned traditional methods or human heuristic approaches. First of all, due to its iterative nature, the disease diagnosis refinement process (Fig. 1) cannot be simply viewed as a classification problem mainly because there is not a clearly defined final classification goal for such a type of process. A pathologist keeps diagnosis refinement (into disease class, subclass, grade, therapeutic target etc) as long as he or she can obtain new information. Second of all, in such a diagnostic workflow, there is not a fixed feature space. In this work, we use feature, fact, factor, and test interchangeably. Instead, the process is based on a dynamic set of facts at hand. Depending on the diagnostic path, the process may require a different set of tests to be conducted. Thirdly, pathologists often use different tests to reveal the same biological mechanisms (e.g., proliferation of B-cells 
can be identified by a test for CD20 or CD23 antigen). Therefore, the IHC panel design philosophy is a complex mental process aimed at constructing a comprehensive but limited set of IHC tests that can generate valuable information at each diagnostic refinement step.

The pathology community needs a robust computational tool that can propose a preliminary diagnostic path and dynamically suggest a set of IHC tests to be performed.

To answer this need, we have developed a novel method that helps to streamline IHC studies by dynamically suggesting an efficient set of IHC tests at each step of the pathology workflow. Our approach, while not entirely new computationally, is a novel application of information entropies and Bayesian probabilities in IHC that can significantly improve speed of IHC diagnostic workflow and reduce intra- and interpathologist variability. Our tool also helps to visualize possible diagnostic paths, which brings a great deal of clarity into the diagnostic process.

\subsection{Methods}

\subsubsection{Dataset}

The data in our work is represented by a matrix of antigen expression profiles $(M A E P)$ across multiple diseases. In general, this type of matrix can be constructed from existing medical literature for specific diseases. Table 2 shows a subset of the matrix used in this study. 
Table 2 Excerpt from a matrix of antigen expression scores across multiple diseases

\begin{tabular}{l|l|l|l|l|}
\hline & \multicolumn{5}{|c|}{ Antigens (ant expr|probability) } \\
\hline Disease|Inc Rate & CD11c & CD15 & CD19 & CD20 \\
\hline MCL|0.51 & $-\mid 0.95$ & $-\mid 0.95$ & $+\mid 0.95$ & $+\mid 0.95$ \\
\hline CLL/SLL|5.17 & $+\mid 0.95$ & ND & $+\mid 0.95$ & $+\mid 0.95$ \\
\hline FL|3.18 & $-\mid 0.95$ & $-\mid 0.95$ & $+\mid 0.95$ & $+\mid 0.95$ \\
\hline MALT|2.35 & $+\mid 0.75$ & $-\mid 0.95$ & $+\mid 0.95$ & $-\mid 0.95$ \\
\hline MZL nodal|0.66 & $-\mid 0.95$ & $-\mid 0.95$ & $+\mid 0.95$ & $+\mid 0.95$ \\
\hline MZL splenic|0.31 & $-\mid 0.95$ & $-\mid 0.95$ & $+\mid 0.95$ & $+\mid 0.95$ \\
\hline LPL/WM|0.62 & ND & $-\mid 0.95$ & $+\mid 0.95$ & $+\mid 0.95$ \\
\hline HCL|0.33 & $+\mid 0.95$ & $-\mid 0.95$ & ND & $+\mid 0.95$ \\
\hline PCM|5.86 & $-\mid 0.95$ & $-\mid 0.95$ & $-\mid 0.95$ & $+\mid 0.75$ \\
\hline Burkitt|0.3 & $-\mid 0.95$ & $-\mid 0.95$ & $+\mid 0.95$ & $+\mid 0.95$ \\
\hline DLBCL GC|3.21 & $-\mid 0.95$ & $-\mid 0.95$ & $+\mid 0.95$ & $+\mid 0.95$ \\
\hline DLBCL Non-GC|3.93 & $-\mid 0.95$ & $-\mid 0.95$ & $+\mid 0.95$ & $+\mid 0.95$ \\
\hline ALCL ALK +|0.25 & ND & $-\mid 0.95$ & ND & $-\mid 0.95$ \\
\hline MF|0.52 & ND & $-\mid 0.95$ & $-\mid 0.95$ & $-\mid 0.95$ \\
\hline B-ALL/LBL|0.76 & ND & $-\mid 0.95$ & $+\mid 0.95$ & $-\mid 0.75$ \\
\hline T-ALL/LBL|0.22 & ND & $-\mid 0.95$ & ND & $-\mid 0.95$ \\
\hline PTCL NOS|0.3 & ND & $-\mid 0.95$ & $-\mid 0.95$ & $-\mid 0.95$ \\
\hline AlLT|0.05 & ND & $-\mid 0.95$ & $-\mid 0.95$ & $+\mid 0.95$ \\
\hline CHL|2.59 & ND & $+\mid 0.95$ & ND & $-\mid 0.75$ \\
\hline NLPHL|0.08 & ND & $-\mid 0.95$ & ND & $+\mid 0.95$ \\
\hline & & & & \\
\hline
\end{tabular}

Rows and columns of this matrix $A$ represent correspondingly disease immunotypic profiles across multiple antigens and antigen expression profiles across multiple diseases. Each row starts with the name of a disease followed, after a vertical bar, by that disease's incidence rate. Disease incidence rates for each malignancy were obtained from the Cancer Statistics Review data provided by the Surveillance Epidemiology and End Results (SEER) website, maintained by the National Cancer Institute [81]. We have to mention here that to tune ATOS to geographically local disease incidents rates, the data from regional cancer registries can be used. An element $A_{i, j}$ of the matrix gives information about expression of the antigen $j$ in disease $i$ and probability of getting such expression value. In the tests reported here, the antigen expression scores and their 
probabilities were derived using primarily studies by Higgins et al. [27] and the WHO disease classification [67] for several families of non-Hodgkin and Hodgkin lymphoma. For simplicity and clarity purposes, only two discrete levels for antigens expression were considered in this paper: expressed ("+") and not expressed ("-"). Antigen is used interchangeably with diagnostic factor or just simply factor and antigen test is interchangeable with antibody test.

\subsubsection{Antigen tree induction algorithm}

Our method extends Shannon's information entropies [82] and Bayesian probabilities to iteratively build an efficient antigen selection tree (EAS-Tree) using a matrix of antigen expression profiles (Section 2.2.1). EAS-Tree can then be used by pathologists to streamline the antibody testing process. The main difference between a traditional decision tree (DT) approach [83], and our EAS-Tree is that a DT is usually used to confirm a single path of antigen expressions while an EAS-Tree is used to cover a comprehensive set of diseases and then rule out irrelevant ones. Moreover, a DT utilizing the entropy minimization concept likely will result in a skewed and deep tree. Such a tree likely will require a large number of antibody tests to provide conclusive recommendations to the pathologists. Unlike the DT approach, the EAS-Tree seeks a balanced tree structure, which suggests to pathologists a small number of antibody tests using an entropy maximization approach. We believe that our method strives to closely mimic the reasoning process of pathologists. To explain the ideas behind this, let us use the following $M A E P$ corresponding to differential diagnoses of which the listed five diseases have equal possibilities for diagnosis (i.e. equal incidence rates) (Table 3) 
Table 3 An example MAEP: “+” expressed, “-” not expressed

\begin{tabular}{lcc}
\hline & Antigen A & Antigen B \\
\hline Disease 1 & + & + \\
Disease 2 & - & + \\
Disease 3 & - & - \\
Disease 4 & - & - \\
Disease 5 & - & - \\
\hline
\end{tabular}

From this example, Antigen $A$ is expressed in only Disease 1. Intuitively, a pathologist would want to test for an expression of Antigen A because if the test comes out positive the diagnostic process would quickly end (with Disease 1 as diagnosis in his/her mind). However, if we assume that no other factors can cause an expression of Antigen $A$, the probability of getting positive expression of Antigen $A$ is $1 / 5$, while the probability of getting negative expression results is $4 / 5$. It means that no matter what the test outcome of Antigen A, it will suggest the following: (1) $20 \%$ chance to confirm Disease 1 (“+” expression) or (2) 80\% chance to rule out only Disease 1 leaving four diseases (“-“" expressions) to be checked. From the same example if we choose another antigen, Antigen $B$, which is expressed in two out of five diseases, the test for that antigen in the most likely case would rule out two diseases. As mentioned previously, an efficient diagnostic process should start with a comprehensive list with diseases and quickly rule out as many irrelevant ones as possible. For this reason, the choice of testing Antigen $B$ is over Antigen $A$. We select an antigen $A g_{j}$ with maximal entropy [84], which can be computed by the following formula:

$$
H\left(A g_{j}\right)=-\sum_{l=1}^{M_{j}} P_{A g_{j}}(l) \log _{2} P_{A g_{j}}(l) \quad \text { Eq. } 2
$$


where $P_{A g_{j}}(l)$ is a fraction of diseases in $M A E P$ for which antigen $A g_{j}$ has $l$-th expression level.

The tree induction algorithm builds an EAS-Tree by splitting the current MAEP into partitions, one for every level of expression of a certain antigen. The antigen is then represented by a node with child nodes for each expression level. At each step of the tree induction, the algorithm picks an antigen that splits diseases in current $M A E P_{j}^{l}$ as evenly as possible. To represent the matrix of antigen expression profiles, we use MAEP with super/sub scripts to identify parts of the matrix that were generated during a split by the antigen tree induction step. For example, $M A E P_{j}^{l}$ is a sub-matrix that is resulted from splitting the matrix by $j$-th antigen $A g_{j}$ and consisting of diseases in which $A g_{j}$ has expression value $l$. Fig. 2 lists the pseudo code for the selection of antigen and the procedure of node splitting for EAS-Tree building. 


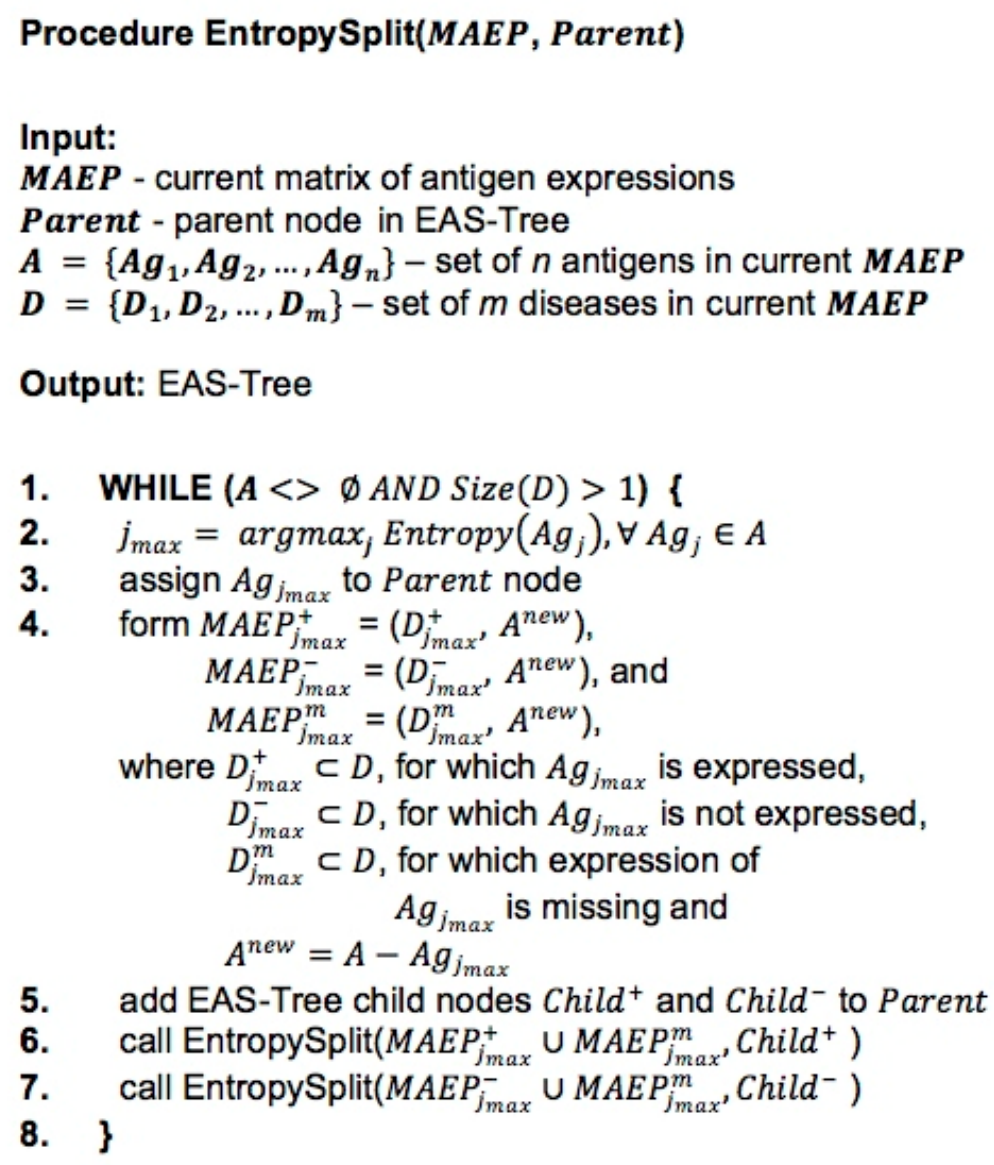

1. WHILE $(A<>\emptyset$ AND Size $(D)>1)\{$

2. $j_{\max }=\operatorname{argmax}_{j}$ Entropy $\left(A g_{j}\right), \forall A g_{j} \in A$

3. assign $A g_{j_{\max }}$ to Parent node

4. form $M A E P_{j_{\max }^{+}}^{+}=\left(D_{j_{\max }^{+}}^{+}, A^{\text {new }}\right)$,

$\operatorname{MAEP}_{j_{\max }^{-}}^{-}=\left(D_{j_{\max }^{-}}^{-} A^{\text {new }}\right)$, and

$M A E P_{j_{\max }^{m}}^{m}=\left(D_{j_{\max }^{m}}^{m}, A^{\text {new }}\right)$,

where $D_{j_{\max }}^{+} \subset D$, for which $A g_{j_{\max }}$ is expressed,

$D_{j_{\max }}^{-} \subset D$, for which $A g_{j_{\max }}$ is not expressed,

$D_{j_{\max }^{m}}^{m} \subset D$, for which expression of

$A g_{j_{\max }}$ is missing and

$$
A^{\text {new }}=A-A g_{j_{\max }}
$$

5. add EAS-Tree child nodes Child $^{+}$and Child $^{-}$to Parent

6. call EntropySplit(MAEP jmax $_{\max }^{+} \cup M A E P_{j_{\max }}^{m}$ Child $\left.^{+}\right)$

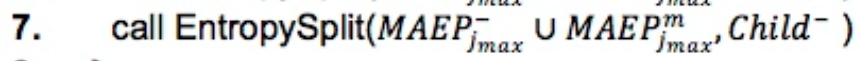

8. \}

\section{Figure 2 Pseudo code of ATOS's EntropySplit() procedure}

Every call of this procedure finds an antigen in $M A E P$ with the maximum entropy (Fig. 2, line 2), splits current $M A E P$ using that antigen (Fig. 2, line 4) and creates new child nodes in EAS-Tree (Fig. 2, line 5). The antigen with maximum entropy splits current $M A E P$ into $l$ partitions, one per expression level of the splitting antigen $(l=2$ in this dissertation). For every resulting MAEP partition, the EntropySplit() procedure calls itself recursively (Fig. 2, lines 6 and 7) and the process repeats. The algorithm stops when there are no available antigens or there is only one disease profile left in the database (Fig. 2, line 1). 
Since antigen expression information for some diseases might not be available, MAEP may have missing values. In our implementation of ATOS we do not impute missing expression values. After splitting MAEP into partitions, diseases for which expression value of the splitting antigen is not available are grouped together in $M A E P_{j_{\max }}^{m}$ (Fig. 2, line 4) and passed to the next recursive calls of EntropySplit() (Fig. 2, lines 6 and 7).

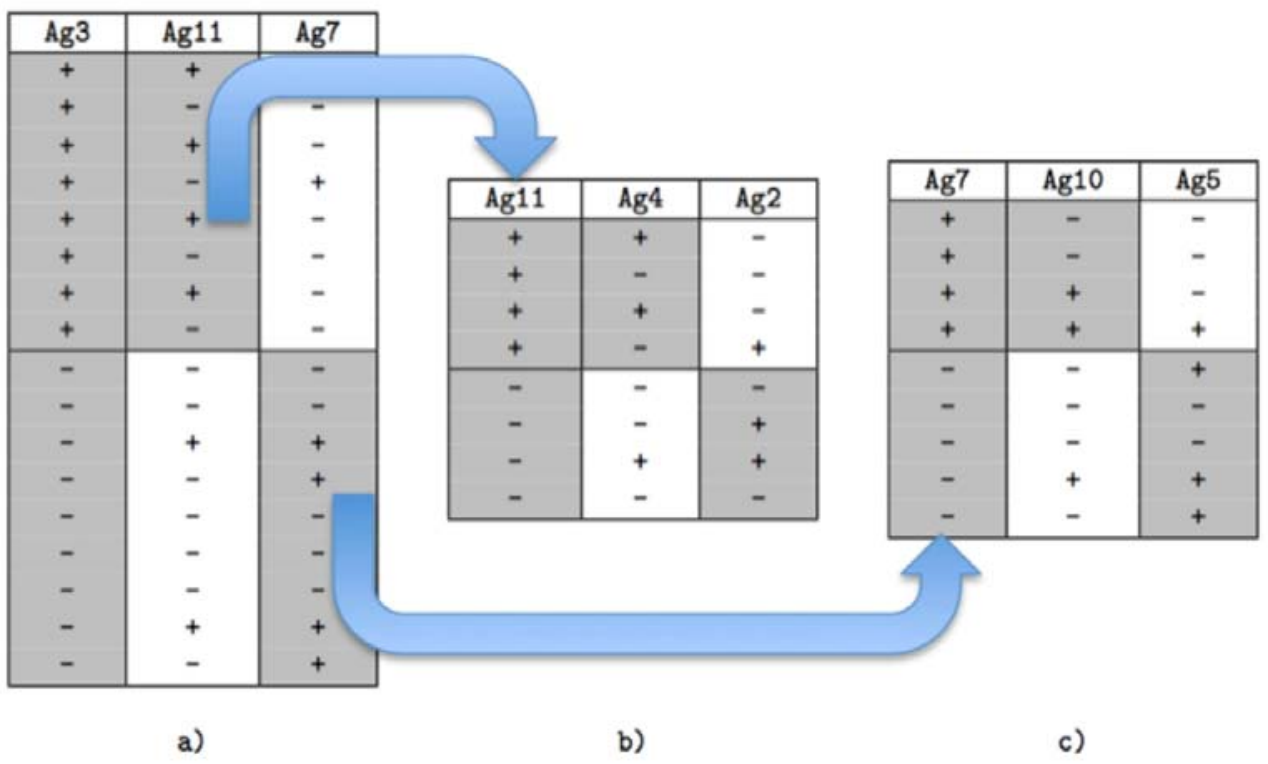

Figure 3 An example of splitting of MAEP

In Fig. 3 (a) the initial MAEP is shown with 17 diseases and is used to explain the logic of the tree induction process in a recursive way. During the first call, the algorithm selects antigen $\mathrm{Ag}_{3}$ as having the largest entropy value of:

$$
\left(A g_{3}\right)=\left(\frac{8}{17}\right) \log _{2}\left(\frac{17}{8}\right)+\left(\frac{9}{17}\right) \log _{2}\left(\frac{17}{9}\right)=0.997
$$


Indeed, the expression values of $A g_{3}$ almost evenly splits $M A E P$ into two partitions $M A E P_{3}^{+}$and $M A E P_{3}^{-}$, one having diseases with "+" expression values of $\mathrm{Ag}_{3}$ and the other one with "-" expression values. This is illustrated by the leftmost column in Fig. 3(a) where disease rows are grouped by expression values to form partitions. $A g_{3}$ becomes the root of the antigen tree (Fig. 4). For each resulting partition, the tree induction algorithm recursively calls itself by passing $M A E P_{3}^{+}$and $M A E P_{3}^{-}$. At the next level of this recursion, the selected antigens are $A g_{11}$ and $A g_{7}$ for partitions $M A E P_{3}^{+}$and $M A E P_{3}^{-}$respectively (Fig. 3(a)). $A g_{11}$ and $A g_{7}$ are added to the tree as child nodes for $A g_{3}$ (Fig. 2-5). At this step there are four new partitions $M A E P_{11}^{+}$and $M A E P_{11}^{-}$(Fig. 3(b)), $M A E P_{7}^{+}$and $M A E P_{7}^{-}$(Fig. 3(c)). For each of these partitions, the induction procedure calls itself recursively again and then adds new antigen nodes $A g_{4}$ and $A g_{2}$ to their parent node $A g_{11}$ and new nodes $A g_{10}$ and $A g_{5}$ to $A g_{7}$ (Fig. 4).

When the algorithm looks for an antigen that best splits a current partition, it chooses from all antigens with the exception of those that appear in the path to the root antigen node. For example, when the algorithm processes partitions generated by $A g_{4}$, it can choose any available antigen except $A g_{4}, A g_{11}$, or $A g_{3}$, since any of these antigens will have zero entropy for partitions $M A E P_{4}^{+}$and $M A E P_{4}^{-}$(see Fig. 3(a)). However, some antigens may appear in different branches of the antigen tree (see double circled nodes on Fig. 4). 


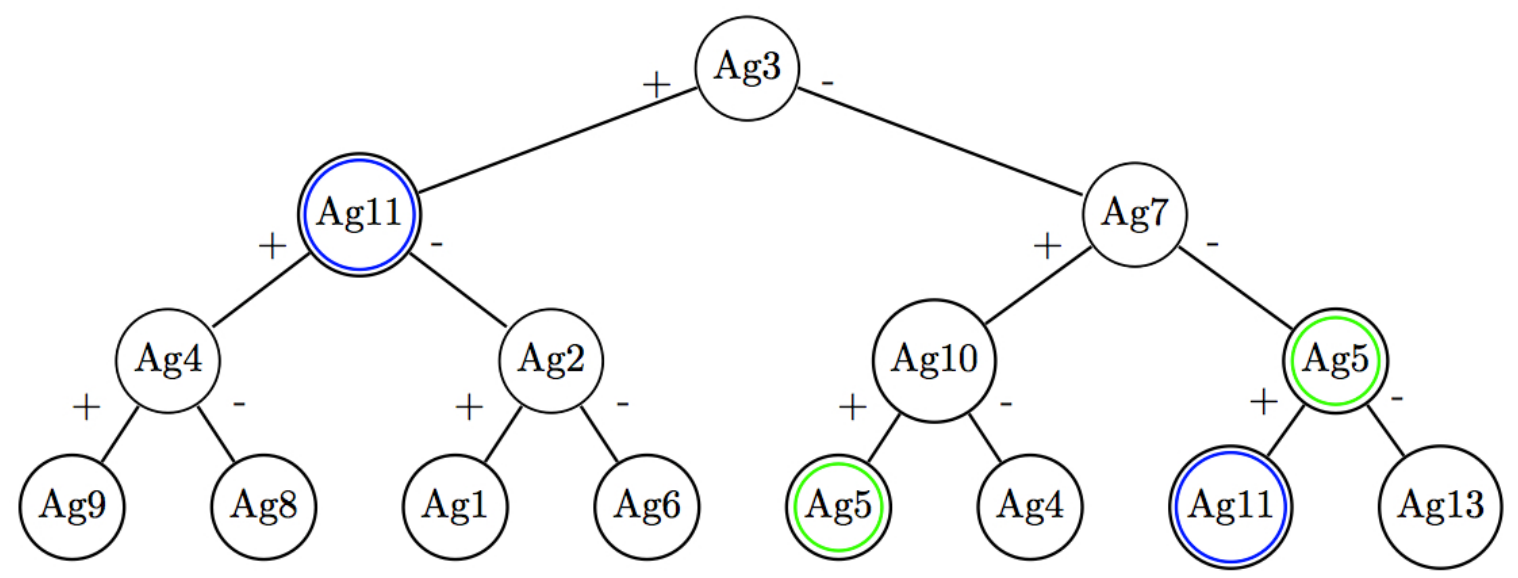

Figure 4 An efficient antigen selection tree (EAS-Tree) based on MAEP from Fig. 3

To reflect a more realistic picture, instead of simply counting occurrences of expression levels over diseases for each antigen in $M A E P$, there are other important parameters to be considered for entropy calculations. During disease diagnosis pathologists usually take into account disease incidence rates. The rationale behind this is that some diseases are prevalent while others are not. A specific antigen expression level is less likely to manifest the presence of a disease with a lower incidence rate than a disease with a high incidence rate. To address this fact we modify entropy computation and include disease incidence statistics in the following way. First of all, the "raw" disease incidence rates, as discussed in Section 2.2.1, have to be normalized. Since the differential diagnosis assumes that the pathology case in question should be diagnosed only with a disease from $M A E P$, we need to make sure that the incidence rates will sum to unity, i.e., $\sum_{i=1}^{N} P_{i n}\left(D_{i}\right)=1$, where $P_{i n}\left(D_{i}\right)$ is a normalized incidence rate for disease $D_{i}$. We compute $P_{\text {in }}\left(D_{i}\right)$ using the following formula: $P_{\text {in }}\left(D_{i}\right)=P_{\text {in }}^{\text {raw }}\left(D_{i}\right) /$ $\sum_{k=1}^{N} P_{\text {in }}^{\text {raw }}\left(D_{k}\right)$. Now the normalized incidence rates can be used as probabilities to 
compute joint probability $P^{*}\left(l_{A g_{j}}, D_{i}\right)$ for a certain expression value $l$ for $j$-th antigen and $i$-th disease:

$$
P^{*}\left(l_{A g_{j}}, D_{i}\right)=P\left(l_{A g_{j}} \mid D_{i}\right) P_{i n}\left(D_{i}\right) \quad \text { Eq. } 3
$$

By summing up all joint probabilities $P^{*}\left(l_{A g_{j}}, D_{i}\right)$ for each level and then normalizing the sums, we obtain marginal probabilities for each expression level $l$ of $j$-th antigen and use them to compute information entropies with the formula:

$$
H\left(A g_{j}\right)=-\sum_{l=1}^{M} P^{*}\left(l_{A g_{j}}, D_{i}\right) \log _{2} P^{*}\left(l_{A g_{j}}, D_{i}\right) \quad \text { Eq. } 4
$$

where the probability takes into account disease incidence rates and the probabilities for antigen expression levels of certain diseases as listed in Table 2.

\subsubsection{Using EAST to guide immunohistochemical studies}

After an EAS-Tree is built, a pathologist can interactively use it as a guidance to conduct actual antibody tests. A pathologist-in-the-loop interface provides a graphical interface for pathologists to select antibody tests and an indicator about the effectiveness of the selection. The outcomes of these tests will then be fed back to the system. If a test for a certain antigen has already been completed, this test will be excluded for future testing. Since ideally each test under any outcome should rule out as many disease diagnoses as possible, the whole testing process should result in an efficient testing workflow. In other words, the testing process using this method, on average, should result in faster turnaround. 
Another way, which resembles the traditional approach (see for instance [27]) is to aggregate nodes of the EAS-Tree into test panels. One of the ways of doing that is to aggregate adjacent levels and construct an antigen panel tree (see Fig. 5). If the number of distinct expression levels $l$ is constant for all antigens in the database (as in our example), the number of antigens in a panel for a given number of aggregated antigen tree levels $K$ equals to $\sum_{l=0}^{K-1} l^{i}$. In the general case, the number of the antigens in panels might be different for each node in the antigen panel tree depending on values of $l$. It has to be noted that while this method does not fully eliminate the redundancy of the expert based IHC classification schemas [27], it can result in substantial decrease in the number of unnecessary tests and significantly reduce inter- and intra- pathologist variability by making the antibody test selection process uniform.

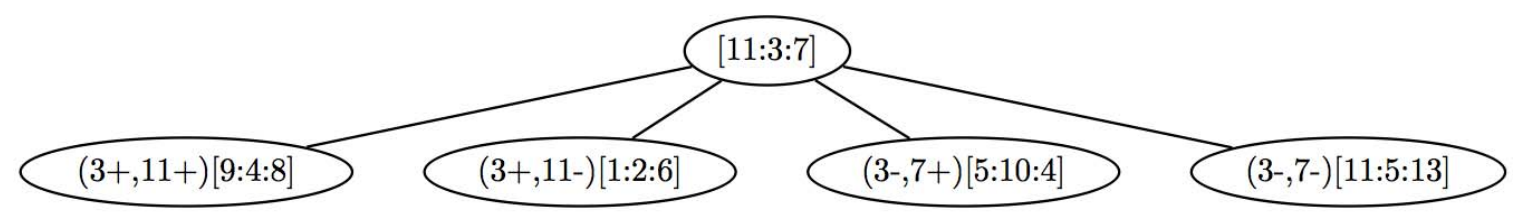

Figure 5 An example of antigen panel tree. The numbers in square brackets identify antigens. The numbers with signs in parentheses indicate outcomes of the testing for corresponding antigens

\subsection{Results and Discussion}

\subsubsection{Method evaluation}

A typical model of the conventional, recommended IHC classification schema for lymphoid tumors was constructed in accordance with text and tables presented in an expert review article [27] as well as WHO guidelines [67]. This model employs two to 
three distinct panels performed in series as determined in conjunction with morphologic features within the tumor. To compare our method with this model, a MAEP for hematopoietic tumors was compiled from the same sources (see Section 2.2). This comprises 20 lymphoma/leukemia cases and 37 antibodies, which represent a relatively broad range of neoplasms and a sizable selection of antibodies. Our first assessment of the quality of the resulting EAS-Tree is to validate nodes from the first few levels with recommendations from the published literature or guidelines.

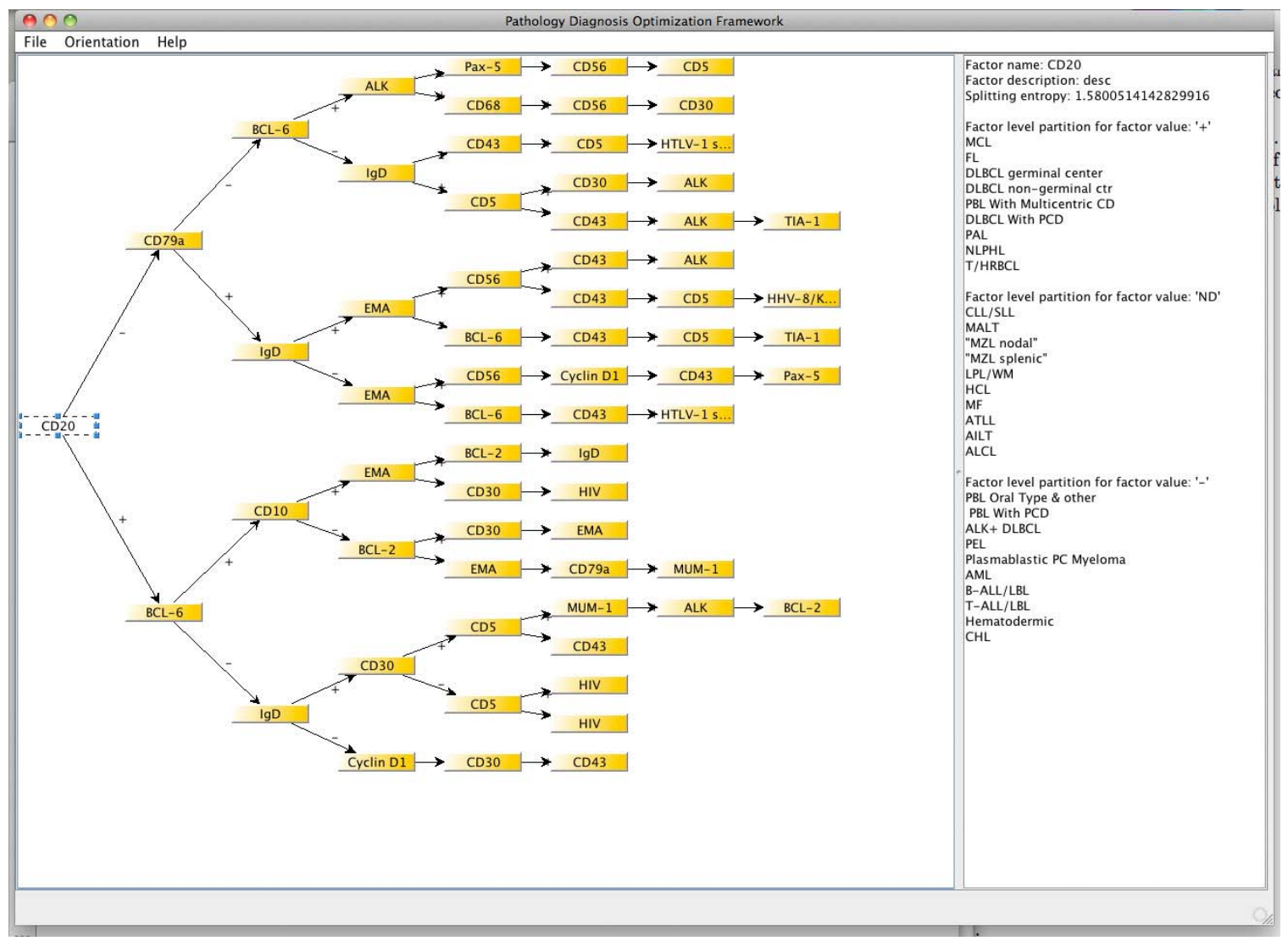

Figure 6 ATOS user interface for diagnostic tree building using automatic entropybased probabilistic algorithm 
As shown in Fig. 6, the three antigen nodes from the first two levels of the tree are CD20, CD79a and BCL-6 antigens, which are B-Cell markers and suggested to be analyzed first by guidelines in [27]. This tree would be valuable for pathologists to determine if the case is B-Cell or T-Cell based lymphoma/leukemia. Since the tree is balanced, the method finds the shortest path to the smallest subset of disease diagnosis candidates. In the worst-case scenario, a pathologist would need to test eight antibodies out of total 37 , and in the best test scenario only six.

\subsubsection{Comparison of the conventional model (WHO, experts' guidelines) and entropy based ATOS}

No diagnostic discrepancies were identified using either the conventional model or our method; however, in general, our method's pathways were distinctly shorter for the majority of disease diagnoses. On average, our method requires slightly less than half of the positive markers and less than a third of the negative markers to reach a definitive diagnosis. This brevity is inherent in the algorithm, which is designed to produce the shallowest diagnostic tree. The method stops when the diagnostic branch contains only a single entity. However, if desired, the algorithm could be readily extended to identify any specified number of additional positive or negative confirmatory antigen tests. Table 2 shows a comparison of number of antibody $(\mathrm{AB})$ tests required to reach a diagnostic conclusion using our method (ATOS) and conventional (Conv.) methods [27], [67]. A two-sample T-test, conducted with the assumption of unequal variances, was significant for the difference between mean number of tests in conventional and ATOS panels. For 
positive, negative and total tests the p-values were correspondingly $2.619 \mathrm{e}-04,1.214 \mathrm{e}-10$, and 3.394e-11.

Table 4 Comparison of immunotypic findings for 14 representative lymphomas/leukemias between human expert model (Conv.) and the entropy-based computer algorithm (ATOS) (“+”: positive, “-“: negative outcomes)

\begin{tabular}{cccccc}
\hline Disease & $\begin{array}{c}\text { Conv. } \\
\text { ABs }+\end{array}$ & $\begin{array}{c}\text { Conv. } \\
\text { ABs - }\end{array}$ & $\begin{array}{c}\text { ATOS } \\
\text { ABs }+\end{array}$ & $\begin{array}{c}\text { ATOS } \\
\text { ABs - }\end{array}$ \\
\hline ALCL & 7 & 13 & 4 & 2 \\
MF & 4 & 16 & 1 & 5 \\
MCL & 5 & 10 & 3 & 3 \\
CLL & 5 & 10 & 4 & 2 \\
\hline FL & 5 & 10 & 2 & 4 \\
MALT & 3 & 13 & 3 & 4 \\
\hline HCL & 7 & 9 & 4 & 1 \\
\hline MZL nodal & 5 & 10 & 3 & 3 \\
\hline B-ALL/LBL & 7 & 11 & 2 & 4 \\
\hline T-ALL/LBL & 8 & 13 & 3 & 3 \\
\hline DLBCL GC & 8 & 10 & 5 & 1 \\
\hline DLBCL NGC & 9 & 9 & 4 & 3 \\
\hline CHL & 7 & 8 & 3 & 3 \\
\hline PCM & 2 & 13 & 3 & 3 \\
\hline MEAN & & & & \\
\hline
\end{tabular}


Table 5 Abbreviations of diseases listed in Table 4

\begin{tabular}{ll}
\hline Abbreviation & \multicolumn{1}{c}{ Disease } \\
\hline ALCL & Anaplastic large cell lymphoma \\
\hline MF & Mycosis fungoides \\
MCL & Mantle cell lymphoma \\
\hline CLL & Chronic lymphocytic leukemia \\
FL & Follicular lymphoma \\
MALT & $\begin{array}{l}\text { Mucosa-associated lymphoid tissue } \\
\text { lymphoma }\end{array}$ \\
\hline HCL & Hairy cell leukemia \\
MZL & Marginal zone lymphoma \\
B-ALL/LBL & Acute B lymphoblastic leukemia \\
T-ALL/LBL & Acute T lymphoblastic leukemia \\
DLBCL & Diffuse large B-cell lymphoma \\
CHL & Classic Hodgkin's lymphoma \\
PCM & Plasma cell myeloma \\
\hline
\end{tabular}

\subsubsection{Comparison of manual antibody test selection using ATOS GUI and entropy based ATOS}

For the next series of experiments we have developed a simulator program that uses the MAEP and ATOS GUI and allows a pathologist to build an antigen selection tree manually. The goal was to capture the way each pathologist approaches the task of reducing the number of suspected diagnoses at every step. The program includes a Graphical User Interface (GUI), which makes the process user friendly and helps to retain the logical order of the diagnostic process rather than rely on memory. This interface is shown in Fig. 7. 


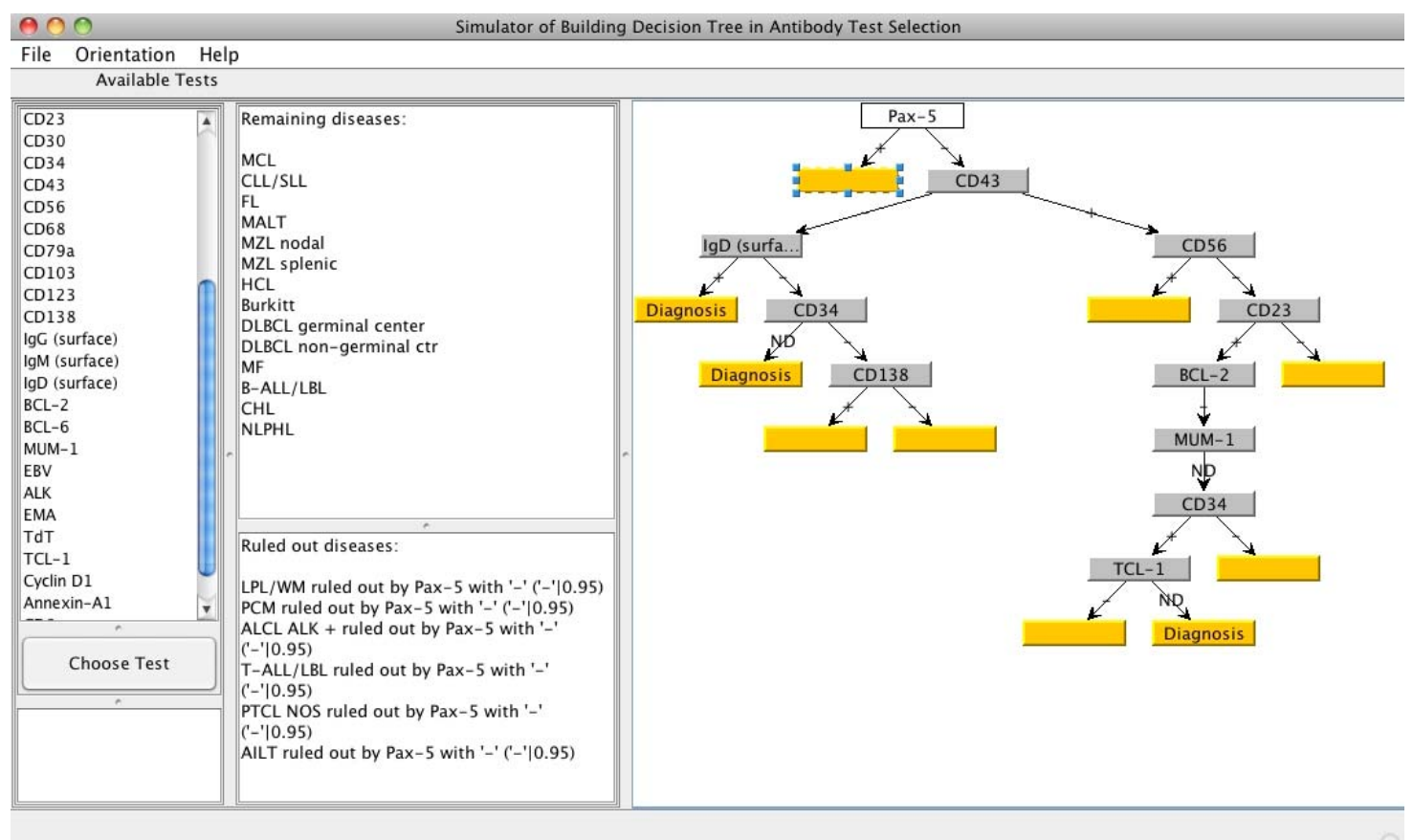

Figure 7 ATOS GUI in simulator program during manual antibody test selection

Moreover, the program provides linkages for the antigen descriptions and their genomic/ proteomic functions derived from well-known online knowledge bases such as UniProtKB/Swiss-Prot [85] and Gene Wiki [86].

Five experienced pathologists participated in the experiments, each with overall experience in practicing anatomic pathology ranging from 7 to 30 years. They were asked to build an antigen selection tree manually. The requirements for an experiment session were to select an antibody test for a given set of remaining (suspected) diseases at each step. The pathologists were not told to use any specific selection criteria; they had to use their own methods. Similar to our entropy-based algorithm, once an antibody test has been used, it could not be used again down in the corresponding decision branch. However, it could be used in a different branch of the tree. 
To perform comparative analysis of the outcomes of manual test selection using the simulator program with experts [27], WHO IHC guidelines [67], and our entropy-based probabilistic algorithm, we plotted a bar chart in Fig. 8 that shows the number of tests required to diagnose each disease (for manual test selection averages across pathologists are shown). Just by using the ATOS-GUI (the simulator program) and the knowledge base of genomic/proteomic functions, the pathologists could significantly improve the antibody test selection process in terms of reducing the overall number of IHC tests required to reach a diagnostic conclusion. On average, the simulator program helps to reduce the number of tests up to twofold, based on comparisons to the expert IHC classification schema and WHO guidelines. Furthermore, by using our entropy-based probabilistic algorithm, we obtain an additional $35 \%$ to $40 \%$ decrease in the number of antibody tests. 


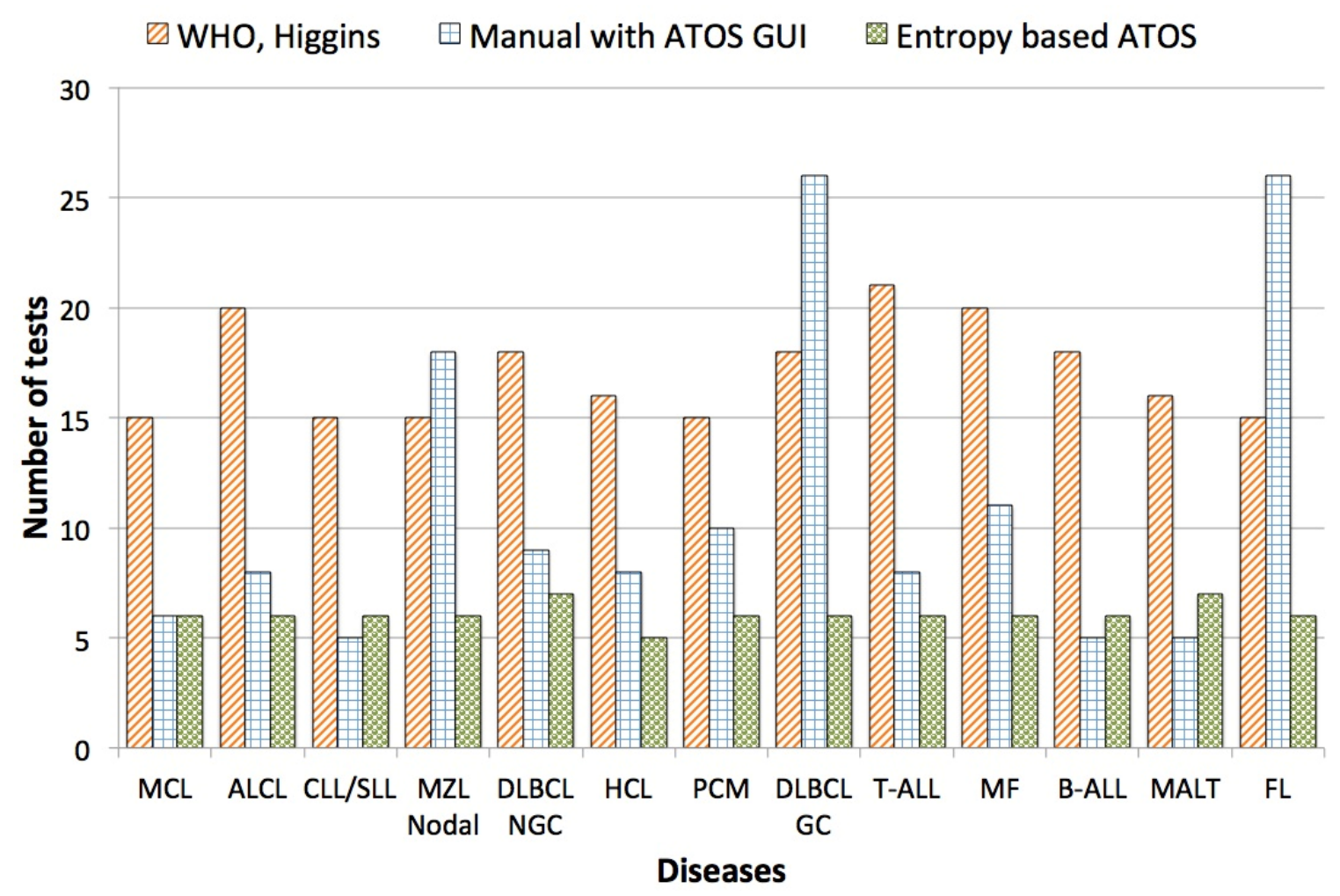

Figure 8 Comparison of conventional test selection (WHO, Higgins), manual test selection using ATOS GUI in simulator program and automatic entropy-based probabilistic method (ATOS-method)

\subsubsection{Inter-pathologist variability}

To measure an inter-pathology variability, we calculated an average difference between numbers of antibody tests required to diagnose the same disease by different pathologists who participated in our research. We then constructed histograms showing the frequency for each such difference (Fig. 9). For example, from the top panel of Fig. 9, we can see that there were three diseases for which pathologists used the same number of tests, five diseases when the number of tests differed on average by 1 , one disease when the number of tests differed by 3, 6 diseases when the number of tests differed by 4 tests 
and so on. The bars appearing on the left side of the histogram correspond to lower variability, while the bars on the right side correspond to the higher variability. We repeated the experiment and plotted another histogram, which is shown on the bottom panel of Fig. 9. It can be seen from the chart that the difference in the number of tests in some cases can reach 10 tests.

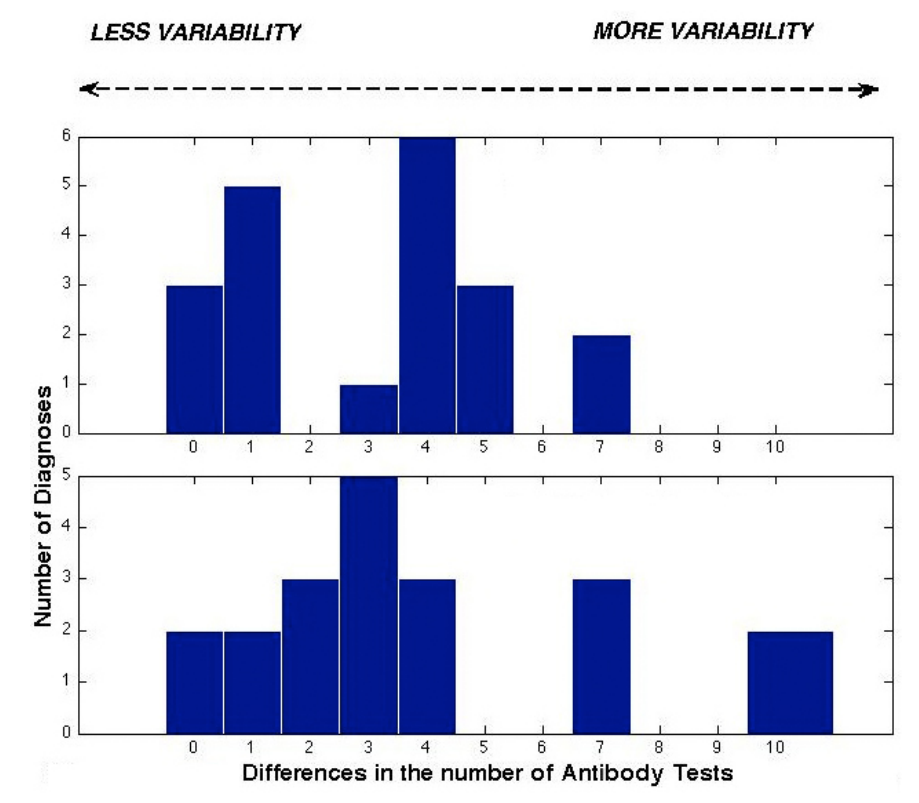

Figure 9 Inter-pathologist variability (two experiments)

The overall level of inter-pathologist variability is significant, showing that the current antibody selection process is not well standardized. Even though the pathologists were aware of the general guidelines for using IHC for malignancies of lymphoid tissue (e.g., WHO [67]), each of them used his or her own heuristics to assemble the diagnostic tree. The use of such tacit knowledge by pathologists during decision-making has been previously discussed in the literature $[87,88]$. Due to consistent tree building by our algorithm based on $M A E P$ obtained by literature, variability using our method is zero in 
all runs to emphasize the benefit of utilizing our entropy-based probabilistic algorithm in light of maintaining low inter-pathologist variability.

\subsubsection{Intra-pathologist variability}

Contrary to our expectation that intra-pathologist variability would be smaller than inter-pathologist variability, interestingly we did not observe that. The overall level of intra-pathologist variability had not been found significantly different from interpathologist variability, based on the analysis of the corresponding histograms (Figures 9 and 10). Studies on other types of cancers have reported similar findings [74]. After further analyses of the EAS-Trees, we concluded that the differences in diagnostic paths generated by the same pathologist can be attributed to the fact that some antigens carry similar functions. The pathologist could have picked a different antigen in place of the one that was no longer available.

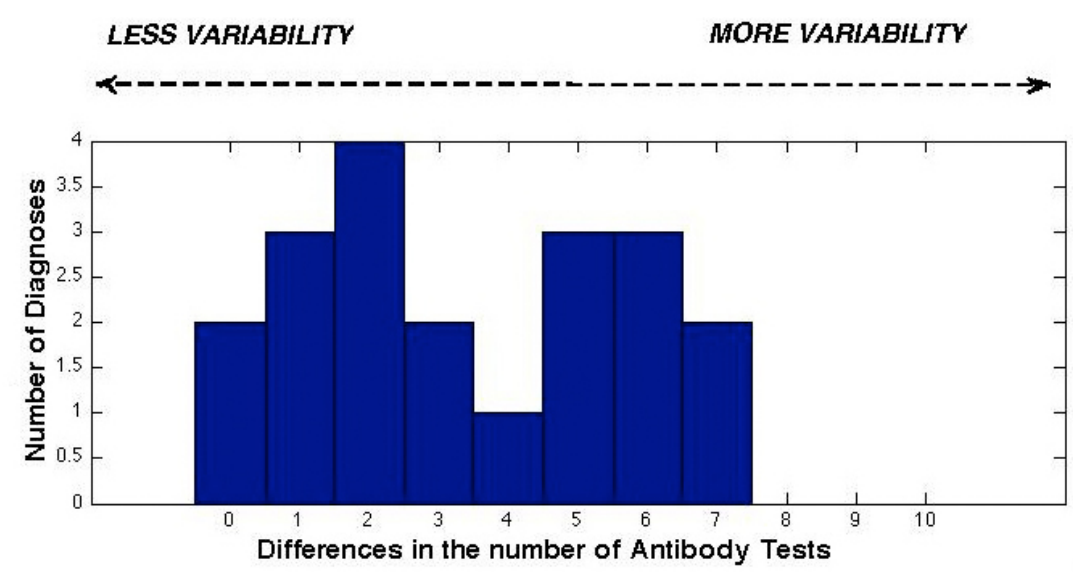

Figure 10 Intra-pathologist variability 


\subsubsection{Experimental clinical study}

To preliminary assess clinical utility of ATOS, we have conducted a small retrospective study. Twenty cases of hematopoietic tumors were selected. The diagnoses of these cases were performed in the past by five different pathologists that used traditional methods to choose IHC tests. The cases were then processed again by three expert hematopathologists in context of this study. They analyzed hematoxylin and eosin (H\&E) slides and came up with differential diagnoses, which then were fed into ATOS. For each case, the number of tests suggested by ATOS that lead to original diagnosis was noted. We then set to answer the following two questions: (1) Is the number of ATOS tests that lead to original diagnosis statistically lower than the number of tests in traditional antibody panels? (2) Can ATOS panel be used by a pathologist to arrive at the proper diagnosis?

To answer the first question we conducted statistical tests using SAS and R tools. Since each case was diagnosed again by three pathologists, a four-sample data setup was established: one traditional and three ATOS. Statistically the question was to compare means of three ATOS samples with the traditional one. Bartlett's, Flinger-Killeen's and Levene's null hypothesis tests showed that the data failed to satisfy homoscedasticity requirement with corresponding p-values $6.474 \mathrm{e}-07,4.133 \mathrm{e}-04$, and $2.000 \mathrm{e}-04$. The normality assumption violation was demonstrated by Shapiro-Wilk, KolmogorovSmirnov, Cramer-von Mises and Anderson-Darling tests (p-values: 0.0001, <0.0100, $<0.0050,<0.0050)$. We therefore decided to use nonparametric tests. The Kruskal-Wallis test was significant (p-value 6.678e-06), which showed overall high level of difference in mean number of tests across all samples. Pairwise differences between each ATOS 
sample and traditional one were measured by Behrens-Fisher and Steel tests. The results were significant with $\mathrm{p}$-values for Behrens-Fisher test for all pairs being less than $5.84 \mathrm{e}$ 06 and for Steel test being less than 3.08e-04. We have, therefore, come to the conclusion that ATOS suggests statistically significantly lower number of tests than the number of tests in traditional IHC panels in real clinical cases.

To answer the second question the ATOS panels of several cases from the study were manually analyzed. This instance of an ATOS algorithm is based on immunohistochemical data derived from a limited literature review [27, 67] and appears to accurately derive the proper diagnosis. Given the clinical setting and $\mathrm{H} \& \mathrm{E}$ findings, the analysis demonstrated the use of redundant or non-contributory stains during original processing of the cases by conventional panels. Several cases illustrated the type of diagnostic situations in which ATOS might be of value in the evaluation of lymphomas. For instance, it would: (1) exclude examination for widely positive antigens; (2) eliminate the implementation of multiple B-cell antibodies simultaneously; (3) avoid the use of stains which are listed as $+/$ - for multiple tumors in the differential; (4) avoid the use of antibodies to evaluate large cell lymphomas when no large cells are present; (5) exclude the application of epithelial-associated antibodies for tumors with a purely lymphoid appearance. Of course, these would be presented as guidelines, not absolute rules for all cases.

The conducted experiments have shown that the pathology diagnostic process can greatly benefit from our new improved methodology for streamlining antibody test selection in IHC studies. One of the most important advantages of our computerized approach is that it is more quantitative than qualitative. For instance, substitution of 
antibody tests may lead to significantly different results because of the subtle differences in expression patterns of corresponding antigens. For example, different B-cell surface expressed proteins can produce slightly different staining in the same disease. A pathologist may not recall and account for that at the time of diagnosis, which can, therefore, complicate the diagnostic process and increase turnaround time. Another problem is related to the fact that many antibodies have been poorly clinically validated [89] and are often selected based on sensitivity only. Due to the low specificity some of the tests do not have sufficient discriminative power and may lead to highly variable results. These issues are accurately handled in our algorithm by the antigen expression probabilities across diseases, which is a rather quantitative task that may not be easily managed by a human. The same applies to the disease incidence rates. It is not a simple task to account accurately for disease incidence rates during diagnosis. Our method provides a solution to these problems, while being flexible enough to allow a pathologist to have full control of the decision process through pathologist-in-the-loop interface.

Our method is very flexible in the sense that it just eliminates diseases that do not support revealed facts. The overall goal of the method is not to suggest a single diagnosis, but to streamline pathology decision-making. For example, in a traditional rule-based expert system, the inference process produces "useful" results only when information about all "key" antecedent terms is entered into the system. If the antecedent part of a rule is only partially satisfied, the rule may not fire and it is hard to extract any benefit from the system in this case. Our method, on the other hand, finds the shortest diagnostic paths using only available facts. It "optimizes" the process by finding the "most efficient" factors at each step (the ones that will lead to ruling out larger number of possible 
diagnoses). The more such facts are available, the more diseases can be ruled out and the leaf nodes of a corresponding EAS-Tree will have fewer possible diagnostic solutions. A pathologist is free to remove from or add diseases/antigens into consideration and the algorithm will dynamically rebuild the EAS-Tree. This gives the pathologist control over the decision process. Moreover, being involved in an interactive decision-making provides the pathologist with better understanding of why one or another disease should be ruled out or ruled in and with what confidence level. ATOS is not one-answer fit system with predefined rules and answers. Based on the differential diagnosis provided by a pathologist, it builds diagnostic trees that reflect the individual practice patterns of that pathologist. Furthermore, the Bayesian probabilistic model used in our method naturally incorporates disease incidence rates as prior probabilities. ATOS can be easily adjusted to account for specific rates of disease occurrences across different demographical and geographical categories.

We share the view that personalized medicine involves the integration of diagnostics and therapeutics into what has become known as theranostics [25]. As such, a more granular sub-classification of diseases based on an increasingly complex proteomic knowledge would be expected to contribute significantly to this process. Granted, our current state of practice of personalized medicine in pathology largely seems to be dependent upon the identification of the expression or activation of proteins that may be unique to an individual's particular cellular pathways rather than representative of a population. Therefore, our algorithm could not be expected to provide much assistance. However, as diagnostics evolves to include pathway-based molecular biological evidence 
(see for instance [90]), new patterns of protein expression may need to be evaluated in which computational support in the manner of this algorithm may be useful.

\subsection{Conclusion}

We have developed ATOS - a novel informatics tool to help pathologists streamline antibody selection process. Using the ATOS-GUI alone, pathologists can significantly speed up antibody selection process. Furthermore, our entropy maximization probabilistic algorithm brings up to $40 \%$ additional decrease in the number of antibody tests required to reach a diagnostic conclusion. Moreover, it significantly reduces inter- and intrapathologist variability, which makes the process more consistent and predictable. A comparative analysis of our method with the World Health Organization classification guidelines and conventional approaches showed that the proposed tool brings approximately a threefold reduction in number of antibody tests required to reach a diagnostic conclusion. Therefore, ATOS has great potential to streamline antibody test selection and significantly decrease costs associated with it. 


\section{Chapter 3: Uncovering Morphoproteomic Relationships Using Probabilistic Graphical Models and RDF Graphs}

In this chapter we introduce a novel method for discovering morphoproteomic relationships using graphical models and public knowledge sources about protein, signal transduction pathways and drugs.

\subsection{Overview}

The heterogeneity of individual patient responses to conventional drug therapies is one of the central problems in personalized medicine and has great impact on clinical outcomes. To address this problem a new field of morphoproteomics was recently introduced [6]. Morphoproteomics is a new method aimed at comprehensive analysis of protein circuitries in diseased cells to design effective drug therapies for individual patient cases. The analysis is done based on the visual expression of antibody-linked (IHC) proteins on histopathological slides.

It has already been a few decades since pathologists began to use IHC to identify antigens of aberrantly expressed proteins - biomarkers of certain diseases. However, this process is often complicated by an inexact nature of protein expression patterns and the overwhelming number of their different combinations that a pathologist has to analyze before coming to a diagnostic conclusion. On the other hand, the vast amounts of information related to cancer genetics, epigenetics, proteomics and other "omics" have been collected and are available in electronic databases. In this dissertation we propose a methodology to automatically "project" protein expression pattern information from the 
histopathological slide into an "omics" knowledgebase in such a way that a pathologist could get a detailed "picture" of the abnormalities in the specimen. It includes not only morphological information of the microanatomy of the tissue, but also information about what proteins are expressed, whether they should be expressed or not, what signaling pathways are triggered and so on. In other words, it enables a pathologist to incorporate molecular knowledge of biochemical pathways into the theranostic process. That could lead to better understanding of high-level physiological processes and allow more precise treatment schemes. We call this approach a "Systems Pathology" paradigm.

The feasibility of this task has been improved by the recent advances in whole slide scanning technology $[76,77,91]$. Several major vendors began to offer scanners that can generate images from whole histopathological slides taken at very high optical magnification [92]. It has become feasible to perform computerized image processing of whole slides in a clinical setting, instead of occasional use of cropped digital images for education or research purposes only. However, there was a need to develop efficient methods to "make sense" of different protein expression patterns. It would be invaluable to use knowledge from high quality knowledgebases to construct morphoproteomic diagrams in an automatic or semi-automatic manner. The computer generated morphoproteomic schemas would have great potential to help identifying inhibitors and cytotoxic agents that can be combined in effective individualized combinatorial therapies. This work introduces such a method that employs probabilistic graphical models for uncovering of relationships between proteins and signal transduction pathways. 


\subsection{Methods}

\subsubsection{Theoretical Foundation}

To infer causal relationships among proteins and pathways, we extend the theory of causation and information relevance, the first comprehensive treatment of which was given by Pearl and Verma [52], followed later by works of Spirtes [53], Verma and Pearl $[93,94]$. The researchers showed that under certain conditions graphical models could be used to represent dependencies and independencies embedded in probability distributions. In particular, Pearl and Verma introduced notions of graphoids.

According to Pearl [95], for probability distribution $F$ over a set of discrete variables $\{a, b, c, \ldots\}$, a graphoid is a relation of the form I $(a, b \mid c)$ that satisfies the following independence conditions:

Symmetry: $\mathrm{I}(\mathrm{X}, \mathrm{Y} \mid \mathrm{Z})<=>\mathrm{I}(\mathrm{Y}, \mathrm{X} \mid \mathrm{Z})$

Decomposition: $\mathrm{I}(\mathrm{X}, \mathrm{Y} \cup \mathrm{W} \mid \mathrm{Z})=>\mathrm{I}(\mathrm{X}, \mathrm{Y} \mid \mathrm{Z}) \wedge \mathrm{I}(\mathrm{X}, \mathrm{W} \mid \mathrm{Z})$

Weak Union: $\mathrm{I}(\mathrm{X}, \mathrm{Y} \cup \mathrm{W} \mid \mathrm{Z})=>\mathrm{I}(\mathrm{X}, \mathrm{Y} \mid \mathrm{Z} \cup \mathrm{W})$

Contraction: $\mathrm{I}(\mathrm{X}, \mathrm{Y} \mid \mathrm{Z}) \& \mathrm{I}(\mathrm{X}, \mathrm{W} \mid \mathrm{Z} \cup \mathrm{Y})=>\mathrm{I}(\mathrm{X}, \mathrm{Y} \cup \mathrm{W} \mid \mathrm{Z})$

Intersection: $\mathrm{I}(\mathrm{X}, \mathrm{Y} \mid \mathrm{Z} \cup \mathrm{W}) \& \mathrm{I}(\mathrm{X}, \mathrm{W} \mid \mathrm{Z} \cup \mathrm{Y}) \Rightarrow \mathrm{I}(\mathrm{X}, \mathrm{Y} \cup \mathrm{W} \mid \mathrm{Z})$,

where $\mathrm{X}, \mathrm{Y}, \mathrm{W}$ and $\mathrm{Z}$ are disjoint subsets of a finite set $\mathrm{U}=\{a, b \ldots\}$ of discrete variables over which $F$ is defined. The Intersection condition is considered only for strictly positive distributions $F$, distributions for which every combinations of events has some chance of being true (for more details see [52]). The graphoid I $(x, y \mid z)$ stands for " $x$ is independent 
of $y$ given $z$ " where $x \in \mathrm{X}, y \in \mathrm{Y}$ and $z \in \mathrm{Z}$. Pearl and Paz [95] introduced the following Completeness Conjecture:

$$
\exists P: P(x, y \mid z)=P(x \mid z) \text { iff } I(x, y \mid z) \quad \text { Eq. } 5
$$

The Completeness Conjecture implies that a set of graphoids is sufficient to specify independencies in probability distributions.

There was, however, an issue of learning and encoding independencies from experimental data. For example, given a set of $n$ measurements (observations) consisting of $m$ binary variables, there could be over $m ! /(2(m-2) !)$ combinations of variables that need to be specified. For each such combination, we need to compute frequencies of variable values in the set of $n$ measurements. For instance, given three variables $a, b$, and $c$, and assuming symmetry property $P(x, y \mid z)=P(y, x \mid z)$ (first axiom on p. 84 in [52]), there will be three distinct conditional probability distributions: $P(a, b \mid c), P(a, c \mid b)$, $P(b, c \mid a)$. For each such distribution we need to compute and store eight different conditional probabilities, e.g., $P(a, b \mid c): P(a=0, b=0 \mid c=0), P(a=0, b=1 \mid c=0), P(a=1, b=0 \mid c=0)$, $P(a=1, b=1 \mid c=0), \quad P(a=0, b=0 \mid c=1), \quad P(a=0, b=1 \mid c=1), \quad P(a=1, b=0 \mid c=1), \quad P(a=1, b=1 \mid c=1)$, resulting in a total of 24 probabilities for all three distributions. Adding one more variable $d$, will increase the number of different conditional probability distributions to 18 : $P(a, b \mid c), \quad \mathrm{P}(a, c \mid b), P(b, c \mid a), P(a, b \mid d), P(a, d \mid b), P(b, d \mid a), P(a, d \mid c), P(a, d \mid c), P(d, c \mid a)$, $P(d, b \mid c), P(d, c \mid b), P(b, c \mid d), P(a, b \mid c, d), P(a, c \mid b, d), P(a, d \mid c, b), P(b, c \mid a, d), P(b, d \mid a, c)$, $P(c, d \mid a, b)$ requiring computation and storage of 144 (8 for each of 18 distributions) conditional probabilities. Further increases to the number of variables will lead to a combinatorial explosion, which makes the task of computing and storing conditional probabilities intractable. 
Using graphs makes learning and encoding of conditional probabilities computationally feasible. Pearl was among the first investigators to demonstrate that a probability distribution can be decomposed into a set of local dependencies using graphical models [52]. He showed that probability of an event could be treated as dependent only on the outcomes of its immediate causes. In the graph terminology it would translate to the statement that the probability of an event associated with a graph node is dependent only on the outcomes of the node's immediate neighbors, the so-called Markov Blanket (MB) nodes for undirected graphs and the Directed Markov Blanket nodes for Directed Acyclic Graphs. A directed graph, $\mathrm{G}=(\mathrm{N}, \mathrm{E})$, with a set of nodes $N$ and a set of edges $E$ is a graph where all edges $E$ are directed:

$$
\forall e_{k}=\left(n_{i}, n_{j}\right) \in E, i<j, n_{i}, n_{j} \in N, k, i, j=1,2 \ldots: e_{k}=\left\{\begin{array}{c}
n_{i} \longrightarrow n_{j} \\
\text { or } \\
n_{i} \leftarrow n_{j}
\end{array} \quad \text { Eq. } 6\right.
$$

where arrows $\rightarrow$ and $\leftarrow$ denote direction of an edge. Graphs for which Eq. 6 does not hold are considered to be undirected. A directed acyclic graph (DAG) is a directed graph that does not have any cycles. A cycle in a directed graph is a path Path $=\left(n_{1}, n_{2} \ldots . n_{m}\right)$, $n_{i} \in N, i=1 \ldots m$ such that $n_{1}=n_{m}$ and $\forall i=1 \ldots m-1: n_{i} \rightarrow n_{i+1}$. Decomposition of a probability distribution $P$ into local dependencies using graphs and MB property requires computation of a significantly smaller subset of all conditional probabilities $P(\cdot \cdot \cdot)$. Therefore, extraction of conditional independencies from these probabilities using Eq. 5 becomes computationally feasible even for large values of $m$. The independence relations themselves are also naturally represented by graphs. For instance, the statement " $x$ is independent of y given $z$ " is equivalent to saying that "node $z$ separates node $x$ from node 
$y$ " in the graph language. That is, for every set of nodes $x, y, z$ of a DAG $G$ and the set of all paths $S_{\text {Path }}=\{\operatorname{Path}(x, y)\}$ from node $x$ to node $y$ we have:

$$
I(x, y \mid z) \equiv \forall \operatorname{Path}(x, y), \operatorname{Path}(x, y) \in S_{\text {Path }}: \mathrm{z} \in \operatorname{Path}(x, y) \quad \text { Eq. } 7
$$

This means there is no edge between nodes $x$ and $y$ and all paths between these nodes should pass through the node $z$.

The structure of a graph that encodes independencies of $P$ can be constructed either manually by domain experts or learned from data. In any case, the resulting probabilistic network along with the set of conditional probabilities can be used to make inferences. Two types of networks were originally introduced: Random Markov Field (RMF) and Bayesian Network (BN) [52]. RMFs use undirected graphs to encode dependencies, whereas BNs use DAGs to do the same. Since DAGs encode directional probabilistic dependencies, they exhibit more expressive power and can be used to infer causal relationships when structure of a network is learned from experimental data. There are two main categories of learning BN structure from data. Score-based structure learning approaches use conditional probabilities for computing scores to assess the "quality" of heuristically generated network structures and to select the best. It is a more quantitative method because it involves manipulation of numerical parameters of the probability distribution $P$. Constraint-based structure learning uses independence relations directly to infer the structure of the network.

In this work we extend PGM formalisms of inferring casual relationships from observed data to learn complex relationships among proteins and pathways using $R D F$ graphs of publicly available biological knowledgebases. Representing information from 
the knowledgebases as RDF graphs allows us to induce conditional independence statements (graphoids) $\mathrm{I}(\cdot, \cdot \cdot)$ that reflect biological dependencies. The graphoids are then fed into constrained-based structure learning algorithms to learn casual relationships. Fig. 11 illustrates the difference between our and traditional approaches of inferring causation. In a probabilistic setting the graphoids are extracted from probability distributions (gray workflow arrows in Fig. 11), while in our method we use RDF graphs to do so (black workflow arrows in Fig. 11). In Section 3.3 .4 we examine the benefits of using knowledgebases instead of experimental data that can be of great importance to the biomedical research in light of uncovering biological mechanisms.

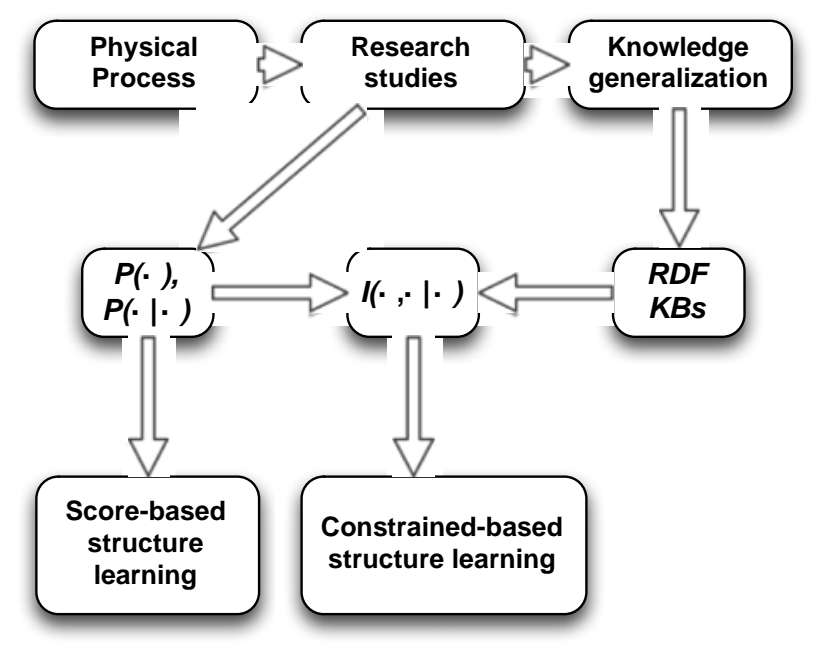

Figure 11 Workflow for inferring casual relationships

In Section 3.2.2 we show how to induce sets of graphoids by translating the definition of conditional independence to be applicable to RDF graphs. 


\subsubsection{Conditional Independence in RDF Graphs}

The theory of causation revolves around the notion of information relevance, which in traditional probabilistic systems is expressed through the mechanism of conditional probabilities [52] (gray workflow arrows in Fig. 11). To understand that, we note that in majority of cases humans use causation as an "artificial" construct that helps them to reason [96]. If for instance the outcome of event $y$ does not bring any new information about the outcome of event $x$, given that we know the outcome of event $z$, we say that event $y$ is irrelevant to event $x$, since event $z$ already captures all information that can influence on $x$, in other words $P(x, y \mid z)=P(x \mid z)$. In such a case we can say that $y$ is not a direct cause of $x$ or $x$ is independent of $y$ given $z$ : $\mathrm{I}(x, y \mid z)$. With knowledgebases we do not have any probabilistic information that can be used to derive independencies $\mathrm{I}(x, y \mid z)$ through Completeness Conjecture (Eq. 5). However, as will be shown in this section, we can "extract" conditional independencies I $(\cdot, \cdot \cdot)$ from the knowledgebases directly if we represent them as RDF graphs.

Since the elementary unit of information in RDF format is a subject-predicate-object triple, where each object can in turn be the subject of another triple (and vice versa), a knowledgebase in RDF format forms a graph. We call it an RDF graph, with nodes representing RDF subjects and objects and edges representing RDF predicates. Unfortunately, the RDF graph of a knowledgebase cannot directly represent causal relationships for at least two reasons: (a) not all edges (RDF predicates) can be considered as reflecting causal relationships, and (b) there are many nodes that have no relevance to the domain in question but rather are introduced to maintain organizational structure of the knowledgebase. 
In this work, we propose using $\mathrm{RDF}$ graphs of knowledgebases only to extract conditional independencies or graphoids $\mathrm{I}(\cdot, \cdot \cdot)$. The graphoids can then be used to generate probabilistic graphical models (e.g. Bayesian Networks) with causal links among entities of interest (Fig. 12).

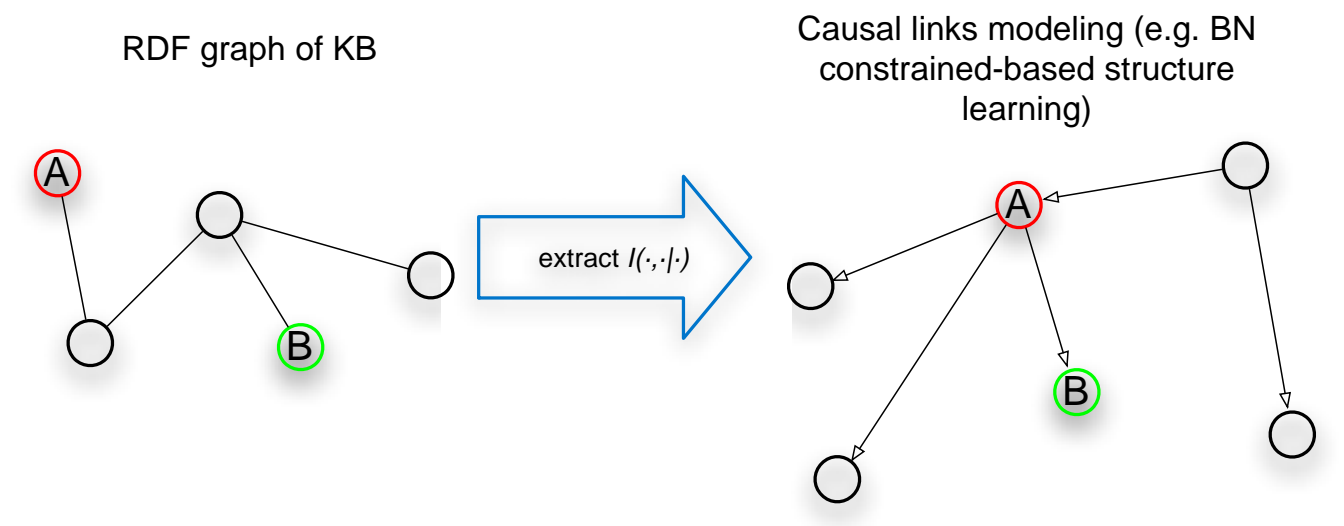

Figure 12 The proposed schema of inferring causal links

Before defining conditional independencies in RDF graphs, this chapter will formally introduce basic concepts. In this work we will use simplified definitions for RDF knowledgebase and ontology.

An ontology $O=\{C, R\}$ is a set of concepts $C$ along with a set of relations $R$ over those concepts. In this research, concepts $C$ include such biological entities such as genes, proteins, protein complexes, biochemical reactions, signal transduction pathways and others. Examples of relations $R$ among concepts $C$ are "COFACTOR" and "PATHWAYCOMPONENTS". This research is concerned with a special type of relations R called object properties $R_{O}$, which is a subset of all relations $R$. 
An RDF knowledgebase $K B=\left\{T, S_{R S}, S_{O}\right\}$ is a set of RDF triples $T$, RDF resources $S_{R S}$ and a set of ontologies $S_{O}$. The set of all RDF resources $S_{R S}=\left\{R S_{O}, L\right\}$ consists of a set of object resources $R S_{O}$ and a set of literals $L$. An object resource $r S_{O} \in R S_{O}$ is an instance of a concept from the corresponding ontology $O \in S_{O}$. For example, for a concept protein from ontology $O$ there can be an $\mathrm{RDF}$ object resource AKT1. Literals $L$ include string constants and numbers. Each RDF resource $r s \in S_{R S}$ and relation $r \in R$ from a set of ontologies $S_{O}$ can appear in $K B$ multiple times in so-called RDF triples. An RDF triple $t \in T$ is a relation of the form $t=\left(r S^{S}, r^{P}, r s^{O}\right)$, where $r s^{S}, r^{P}$ and $r s^{O}$ are called subject, predicate and object respectively. Now we can give a formal definition for an RDF graph.

Definition (RDF graph): Given an RDF knowledgebase $K B=\left\{T, S_{R S}, S_{O}\right\}$ and isomorphic mapping functions $f(n)$ and $f e()$, an $R D F$ induced graph or simply an $R D F$ graph is a graph $\mathrm{G}_{\mathrm{RDF}}=\left\{\mathrm{N}_{\mathrm{RDF}}, \mathrm{E}_{\mathrm{RDF}}\right\}$ with set of nodes, $\mathrm{N}_{\mathrm{RDF}}$, and set edges, $\mathrm{E}_{\mathrm{RDF}}$, for which the following holds true:

$$
\begin{gathered}
\forall t=\left(r s^{S}, r^{P}, r s^{O}\right) \in T: \\
\exists n_{i} \in N_{R D F}: n_{i}=f n\left(r s^{S}\right) \wedge \\
\exists n_{j} \in N_{R D F}: n_{j}=f n\left(r s^{O}\right) \wedge \\
\exists e\left(n_{i}, n_{j}\right) \in E_{R D F}: e=f e\left(r^{P}\right)
\end{gathered}
$$

Eq. 8

In context of morphoproteomics we will work with RDF knowledgebases of proteins, protein complexes, and pathways. Every protein $p r$, protein complex prc and pathway $p w$ is modeled by an object resource $r s_{O} \in R S_{O}$ in $\mathrm{RDF}$ knowledgebase $\mathrm{KB}$ and a corresponding node $n_{\mathrm{i}} \in \mathrm{N}_{\mathrm{RDF}}$ in $\mathrm{RDF}$ graph $\mathrm{G}_{\mathrm{RDF}}$. A protein complex prc consists of 
proteins $p r$, i.e., $p r c=\left\{p r_{i}\right\}, i=1 \ldots m_{\mathrm{PR}}$, and every pathway $p w$ consists of proteins, protein complexes, and other pathways (which we call sub-pathways): $p w=\left\{p r_{i}, p r c_{j}\right.$, $\left.p w_{k}\right\}, i=1 \ldots m_{\mathrm{PR}}, j=1 \ldots m_{\mathrm{PRC}}, k=1 \ldots m_{\mathrm{PW}}$.

Next, let us define a state function $a()$. The state function $a()$ is defined for proteins $p r$, protein complexes prc and pathways $p w$ and returns a state of these biological entities. This research considers only two states for every pr, prc, and $p w$ : 1 or active and 0 or inactive. The biological meaning of active state of a protein $p r$ is a condition where the protein $p r$ is expressed, i.e., present in quantities sufficient to carry out its functions. Below are formal definitions for active states for proteins $p r$, protein complexes $p r c$ and pathways $p w$ :

$$
a(p r)=1 \text { if } q(p r) \geq T_{f}
$$

where functions $q()$ returns quantity of protein $p r$ and $T_{f}$ is the minimum amount of protein $p r$ needed for it to carry out its functions. Then for every protein complex prc we have:

$$
a(p r c)=1 \text { if } \forall p r_{i}, p r_{i} \in p r c: a(p r)=1
$$

and for all pathways $p w$ we have:

$$
\begin{gathered}
a(p w)=1 \text { if } \forall p r_{i}, p r_{i} \in p w: a\left(p r_{i}\right)=1 \wedge \\
\forall p r c_{j}, p r c_{j} \in p w: a\left(p r c_{j}\right)=1 \wedge \\
\forall p w_{k}, p w_{k} \in p w: a\left(p w_{k}\right)=1
\end{gathered}
$$

We define conditional independence of nodes in an RDF graph $G$ of a knowledgebase $K B$ according to some biological ontology $O$ similarly to conditional independence defined by Pearl for DAGs [52]. First, we divide all object properties $R_{O}$ of ontology $O$ 
into two groups: (i) directional and (ii) non-directional RDF predicates, according to some biological interpretation. Like directed edges in Pearl's DAGS, directional RDF predicates reflect possible causal relationship between nodes. In our setting with proteins, protein complexes, and signal transduction pathways, we define a causal relationship as the one that signifies an activation of one biological entity due to the active state of another (possibly through mechanisms of phosphorylation or ubiquitination).

Definition (directional predicate): Given an $\mathrm{RDF}$ graph $\mathrm{G}_{\mathrm{RDF}}=\left\{\mathrm{N}_{\mathrm{RDF}}, \mathrm{E}_{\mathrm{RDF}}\right\}$ induced from an RDF knowledgebase $K B=\left\{T, S_{R S}, S_{O}\right\}$ with a set of object properties $R_{O}=\left\{r_{O}\right\}$ for each ontology $O \in S_{O}$, an isomorphic mapping function $f\left(\right.$ ), and state function $a(), r_{O}$ is a directional predicate if the following holds:

$$
\begin{aligned}
\forall e\left(n_{i}, n_{j}\right)= & f\left(r_{O}\right), e \in E_{R D F}, r_{O} \in R_{O}, n_{i}, n_{j} \in N_{R D F}, i \neq j: \\
& \left\{\begin{array}{l}
\text { if } a\left(n_{i}\right)=1 \text { then } a\left(n_{j}\right)=1 \\
\text { or } \\
\text { if } a\left(n_{j}\right)=1 \text { then } a\left(n_{i}\right)=1
\end{array}\right.
\end{aligned}
$$

All other object properties $R_{O}$ of ontology $O$ for which Eq. 3.2.2.5 does not hold are considered to be unidirectional predicates. Examples of directional predicates are object properties "CONTROLLER" and "CONTROLLED" of the BioPax 2 ontology [97], which can be viewed as reflecting causal relationships. Examples of non-directional predicates are "PATHWAY-COMPONENTS" and "PHYSICAL-ENTITY." While these object properties cannot be considered as representing causation, they do indicate other types of relations between entities in $K B$ (e.g., correlation of protein expressions, wholepart relationships). 
All nodes in RDF graphs are classified as colliders and non-colliders. A directional predicate has a head and a tail. The tail is connected to an RDF node, whose corresponding biological entity's active state causes an RDF node connected to a head of the directional predicate to also switch to the active state. If three RDF nodes are connected by directional predicates and the middle RDF node is connected to the heads of both directional predicates, this middle node is called a collider.

A set of RDF nodes $\left(n_{1}, n_{2} \ldots . n_{m}\right), n_{i} \in N_{\mathrm{RDF}}, i=1 \ldots m$ forms an $R D F$ path Path $_{\mathrm{RDF}}=$ $\left(n_{1}, n_{2} \ldots . n_{m}\right), n_{i} \in N, i=1 \ldots m$ if $\forall i=1 \ldots m-1: n_{i} \rightarrow n_{i+1}$ or $n_{i}-n_{i+1}$ or $n_{i} \leftarrow n_{i+1}$, where $\rightarrow$ and $\leftarrow$ denotes directional predicates and — denotes unidirectional predicates. An RDF path Path $_{\mathrm{RDF}}$ is called a non-collider path if none of its nodes is a collider, and an RDF path Path $_{\mathrm{RDF}}$ is called a collider path if it contains a least one collider node. An active path between nodes $\mathrm{A}$ and $\mathrm{B}$ is the one that:

I. For non-collider path, there are no nodes between A and B that are members of S;

II. For each collider node in a collider path, the collider itself or any of its descendants is a member of $\mathrm{S}$. 


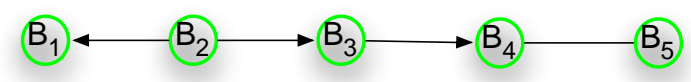

Figure 13 An RDF path without colliders

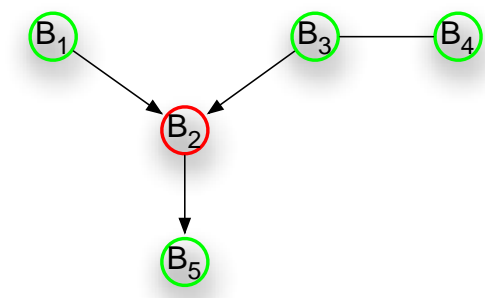

Figure 14 An RDF path with a collider node $B_{2}$

Fig. 13 shows a path in RDF graph (from now on an RDF path) that does not have any colliders. If neither of the nodes $\mathrm{B}_{2}, \mathrm{~B}_{3}$ or $\mathrm{B}_{4}$ is a member of the set $\mathrm{S}$, then the path is considered to be active, which means nodes $\mathrm{B}_{1}$ and $\mathrm{B}_{5}$ are not independent given the set $\mathrm{S}$. Conversely, if some of the nodes $\mathrm{B}_{2}, \mathrm{~B}_{3}$ or $\mathrm{B}_{4}$ are members of the set $\mathrm{S}$, then the path is considered to be inactive, which means nodes $\mathrm{B}_{1}$ and $\mathrm{B}_{5}$ are independent given set $\mathrm{S}$ : $\mathrm{I}\left(\mathrm{B}_{1}, \mathrm{~B}_{5} \mid \mathrm{S}\right)$.

Fig. 14 presents an RDF path between nodes $\mathrm{B}_{1}$ and $\mathrm{B}_{4}$ that has a collider node $\mathrm{B}_{2}$, which is sometimes referred in the literature as forming a $v$-structure or a common-effect structure [47]. If the node $\mathrm{B}_{2}$ or its descendant node $\mathrm{B}_{5}$ (or any other possible descendant of the node $B_{2}$ ) is a member of the set $S$, then the path from the node $B_{1}$ to the node $B_{4}$ is active, which means nodes $\mathrm{B}_{1}$ and $\mathrm{B}_{4}$ are not independent given the set $\mathrm{S}$. Conversely, if both node $\mathrm{B}_{2}$ and its descendant node $\mathrm{B}_{5}$ (and all other descendants of the node $\mathrm{B}_{2}$ ) are not members of the set $\mathrm{S}$, then the path is considered to be inactive, which means nodes $\mathrm{B}_{1}$ and $\mathrm{B}_{4}$ are independent given the set $\mathrm{S}$ : $\mathrm{I}\left(\mathrm{B}_{1}, \mathrm{~B}_{4} \mid \mathrm{S}\right)$.

We can now define conditional independence of nodes in RDF graphs as follows: 
Conjecture 1 (Conditional independence in RDF graphs): Node $a$ is independent of node $b$ given a set of nodes $S$ if and only if there exists no active path between nodes $a$ and $b$.

Let us now examine a domain specific interpretation of the Conjecture 1 as it applies to the proteins and signal transduction pathways to see if it can be accepted without a formal proof. We need to take a closer look at how directional and non-directional predicate edges affect conditional independence in both types of RDF paths with and without colliders.

There could be several node-edge-node-edge-node patterns, which we call edge junctions, in an RDF path without colliders. This chapter will review some of these patterns from Fig. 13. All others have either equivalent explanation or can be considered as their combinations. For each pattern, we must ask if the outer nodes are independent given the middle node.

Non-collider path. Pattern 1. In the RDF path from node $\mathrm{B}_{1}$ to node $\mathrm{B}_{3}$, the middle node $\mathrm{B}_{2}$ has adjacent edges that are directional predicates (according to ontology $O$ ) with their heads pointing away from the node $\mathrm{B}_{2}$. This is known as a common cause structure [47]. The protein/pathway $B_{1}$ is active when protein/pathway $B_{2}$ is active because the latter is a direct cause of the former. The protein/pathway node B3 is also in active state because of node $\mathrm{B}_{2}$, which is its direct cause as well. We can therefore conclude that an expression of $\mathrm{B}_{3}$ indirectly indicates an expression of $\mathrm{B}_{1}$ if we do not consider the state of node $\mathrm{B}_{2}$. In other words, we have $\neg \mathrm{I}\left(\mathrm{B}_{1}, \mathrm{~B}_{3} \mid \emptyset\right)$, which is equivalent to saying that the path from $\mathrm{B}_{1}$ to $B_{3}$ is active because separating set $S$ is empty ( $\varnothing$ indicates an empty set) and $B_{2}$ is not in $\mathrm{S}$. However, if we know the expression status of $\mathrm{B}_{2}$, the knowledge of the status of $\mathrm{B}_{3}$ 
becomes irrelevant to the expression of $\mathrm{B}_{1}$. In other words, we have that $\mathrm{I}\left(\mathrm{B}_{1}, \mathrm{~B}_{3} \mid \mathrm{B}_{2}\right)$ which is the same as to say that path $B_{1}$ is $B_{3}$ is inactive because node $B_{2}$ is in the separating set S.

Non-collider path. Pattern 2. The independence rules for Pattern 1 will hold for the RDF path from pathway/protein node $\mathrm{B}_{3}$ to node $\mathrm{B}_{1}$ because of the symmetry.

Non-collider path. Pattern 3. The RDF path from node $\mathrm{B}_{2}$ to node $\mathrm{B}_{4}$ has all directed predicates with a head-to-tail edge junction. This is an example of a causal trail pattern. In this case, expression of protein/pathway node $\mathrm{B}_{4}$ serves as a direct evidence of expression of $B_{2}$, if we have no information of the active status of the protein/pathway $B_{3}$. Therefore $\mathrm{B}_{2}$ and $\mathrm{B}_{4}$ are not marginally independent, i.e., $\neg \mathrm{I}\left(\mathrm{B}_{2}, \mathrm{~B}_{4} \mid \emptyset\right)$, and the path is active. As soon as we have knowledge of the expression state of $\mathrm{B}_{3}$, we will have that $\mathrm{B}_{2}$ and $\mathrm{B}_{4}$ are independent given $\mathrm{B}_{3}$, i.e. $\mathrm{I}\left(\mathrm{B}_{2}, \mathrm{~B}_{4} \mid \mathrm{B}_{3}\right)$ and that the path is inactive, because now $\mathrm{B}_{3}$ serves as evidence of expression of $\mathrm{B}_{2}$ and there is no need to know the active status of protein/pathway $\mathrm{B}_{4}$.

Non-collider path. Pattern 4. If we reverse direction of the path from Pattern 3 and consider the path from protein/pathway node $\mathrm{B}_{4}$ to node $\mathrm{B}_{2}$, we will get a so-called evidential trail pattern. The node $\mathrm{B}_{4}$ will be dependent on the node $\mathrm{B}_{2}$ through a direct cause relationship, given that we do not have information about $\mathrm{B}_{3}$. In other words, the expression $\neg\left(\mathrm{B}_{4}, \mathrm{~B}_{2} \mid \varnothing\right)$ will hold. Once knowledge of the active status of $\mathrm{B}_{3}$ is available, nodes $\mathrm{B}_{4}$ and $\mathrm{B}_{2}$ will be considered to be independent given $\mathrm{B}_{3}$ : $\mathrm{I}\left(\mathrm{B}_{4}, \mathrm{~B}_{2} \mid \mathrm{B}_{3}\right)$.

Non-collider path. Pattern 5 . The RDF path from the protein/pathway node $\mathrm{B}_{3}$ to node $\mathrm{B}_{5}$ has two types of predicates: directional and non-directional. Non-directional predicates, 
as mentioned above, represent dependencies between biological entities that do not have causal underpinning. However, they still indicate other types of relationships between connected entities. Let us consider a case when expression of the protein/pathway node $\mathrm{B}_{4}$ is correlated with the expression of protein/pathway $\mathrm{B}_{5}$. That means that even if the causal relationship between $\mathrm{B}_{4}$ and $\mathrm{B}_{5}$ is unknown, information about the status of the node $\mathrm{B}_{5}$ is relevant to our understanding of what happens to the node $\mathrm{B}_{4}$. We can therefore say that expression of the protein/pathway node $\mathrm{B}_{5}$ is an indirect evidence of the expression status of the node $\mathrm{B}_{3}$, if we do not have any information about active state of $\mathrm{B}_{4}$. We can then state that $\neg \mathrm{I}\left(\mathrm{B}_{3}, \mathrm{~B}_{5} \mid \varnothing\right)$ is true and consider the path to be active. With the knowledge of the active status of $\mathrm{B}_{4}$, we will have that $\mathrm{B}_{3}$ and $\mathrm{B}_{5}$ are independent given $\mathrm{B}_{4}$, i.e. $\mathrm{I}\left(\mathrm{B}_{3}, \mathrm{~B}_{5} \mid \mathrm{B}_{4}\right)$, and the path will become inactive. If nodes $\mathrm{B}_{4}$ and $\mathrm{B}_{5}$ have wholepart relationship, i.e., either protein/pathway $\mathrm{B}_{5}$ is a part of $\mathrm{B}_{4}$ or vice versa; node $\mathrm{B}_{5}$ can still be considered an indirect evidence for the node $\mathrm{B}_{3}$ leading to the same conditional independence properties of the path as in the case of expression correlation. It can be readily shown that other types of non-directional connections will result in the same outcome as for correlation and whole-part relationships.

All other non-collider paths in RDF graphs of protein/pathways $K B$ can either be composed of Patterns 1-5 or be proven in a similar way to possess the same property as Patterns 1-5. For instance if we reverse the direction of predicate between nodes $\mathrm{B}_{4}$ and $\mathrm{B}_{5}$ in Pattern 5, the node $\mathrm{B}_{5}$ becomes an indirect cause of the node $\mathrm{B}_{3}$. We can therefore conclude that for all non-collider paths, the node separation criterion (also known as vertex separation in graphs [52]) defines conditional dependence, which makes Part 1 of Conjecture 1 valid. 
Let us now look at an RDF path with a collider as shown on Fig. 14 where an example of such type of RDF path from protein/pathway node $\mathrm{B}_{1}$ to node $\mathrm{B}_{4}$ has a collider node $\mathrm{B}_{2}$. The protein/pathway associated with the node $\mathrm{B}_{2}$ can be casually influenced by both node $B_{1}$ and node $B_{3}$. For instance if the node $B_{2}$ represents an apoptotic pathway and the $\mathrm{B}_{1}$ and $\mathrm{B}_{3}$ nodes are apoptotic or anti-apoptotic agents, the active status of $\mathrm{B}_{2}$ can be a result of either active status $\mathrm{B}_{1}$ or/and $\mathrm{B}_{3}$. Normally, the expression of agents $\mathrm{B}_{1}$ and $\mathrm{B}_{3}$ might not to be dependent (marginal independence). However, giving the active status of $\mathrm{B}_{2}$ enables us to draw some conclusions about the status of $B_{1}$ depending on the status of $B_{3}$. For example if $B_{2}$ is activated, and we know that $\mathrm{B}_{3}$ is expressed too, there are fewer chances that $\mathrm{B}_{1}$ is expressed than in the case with $\mathrm{B}_{3}$ not expressed. Since the expression of both protein/pathway $\mathrm{B}_{1}$ and $\mathrm{B}_{3}$ nodes can cause expression of $\mathrm{B}_{2}$, knowing that $\mathrm{B}_{3}$ is expressed makes expression of $\mathrm{B}_{1}$ less likely since we have already "explained away" the expression of $\mathrm{B}_{2}$. This case of conditional independence has been previously discussed in the literature [96]. Here, we simply demonstrate that it can be applied to RDF graphs of protein/pathway $K B$ as a conditional independence extraction technique rather than a depiction of conditional independencies of probability distributions by DAGs [47]. We have to add that the existence of a nondirectional link between the $\mathrm{B}_{3}$ and $\mathrm{B}_{4}$ nodes does not change the conditional independence of expression of $\mathrm{B}_{1}$ and $\mathrm{B}_{4}$ proteins/pathways given the status of $\mathrm{B}_{2}$. As we have already mentioned, non-directional predicates do indicate the dependence of expression of one protein/pathway on another (just not casual). Therefore, the status of $\mathrm{B}_{4}$ will "propagate" to $\mathrm{B}_{3}$ and influence the expression of $\mathrm{B}_{1}$ the same way as expression $\mathrm{B}_{3}$ does. In a similar way, since the expression of the $\mathrm{B}_{5}$ protein/pathway is a direct evidence 
of the expression of $\mathrm{B}_{2}$; hence, we can "substitute" node $\mathrm{B}_{2}$ with the node $\mathrm{B}_{5}$ (or any its direct or indirect evidences) and state our conditional dependency of $\mathrm{B}_{1}$ and $\mathrm{B}_{3}$ (or $\mathrm{B}_{4}$ ) given $\mathrm{B}_{5}$ and not $\mathrm{B}_{2}$. It can be concluded now that the Part 2 of Conjecture 1 is valid.

We have now informally shown that the Conjecture 1 can be used to extract conditional independencies from RDF graphs of protein/pathway KBs. Fig. 15 shows an updated diagram from Fig. 11 indicating the functional place of Conjecture 1.

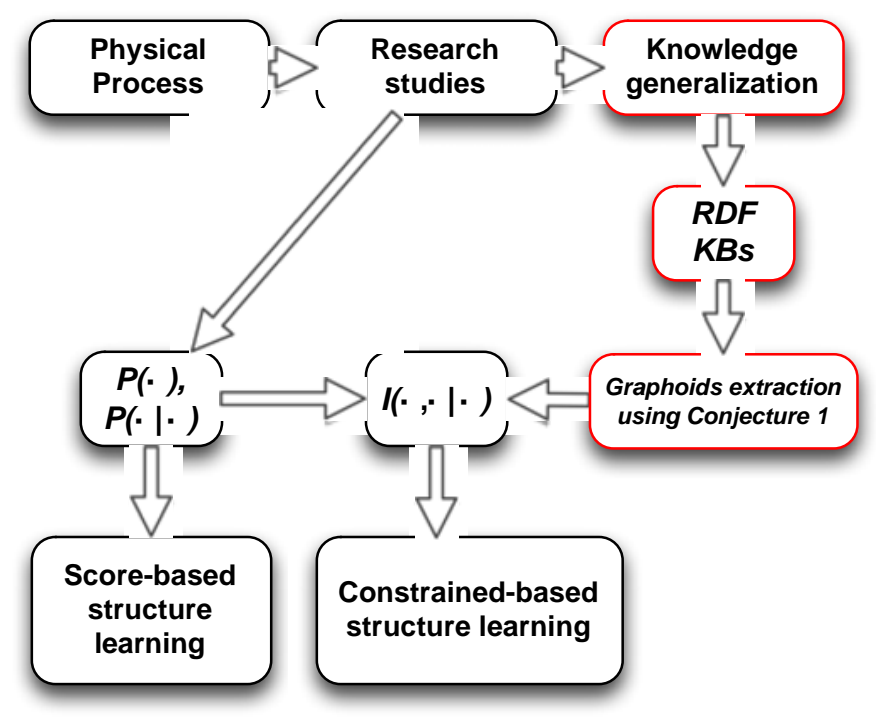

Figure 15 Updated workflow for inferring casual relationships

We formalize the fact that we can use Conjecture 1 to extract independencies as follows:

Conjecture 2 (Extraction of independencies from RDF graphs): If an independence model $M$ (set of graphoids $\mathrm{I}(\cdot, \cdot \cdot)$ ) is embedded in an RDF graph $G$, it can be extracted by iterative application of Conjecture 1. 


\subsubsection{Semantic "Mashup" Knowledgebase of Genetic, Proteomic and Drug Data}

To mine for cause and effect relationships among proteins and pathways using ideas presented in Sections 3.2.1 and 3.2.2 we use a Semantic Web [98] "mashup" knowledge base (SWMKB) that unifies a number of online biomedical databases. The overall schema of the proposed semantic "mashup" is presented in Fig. 16.

\section{SWMKB}

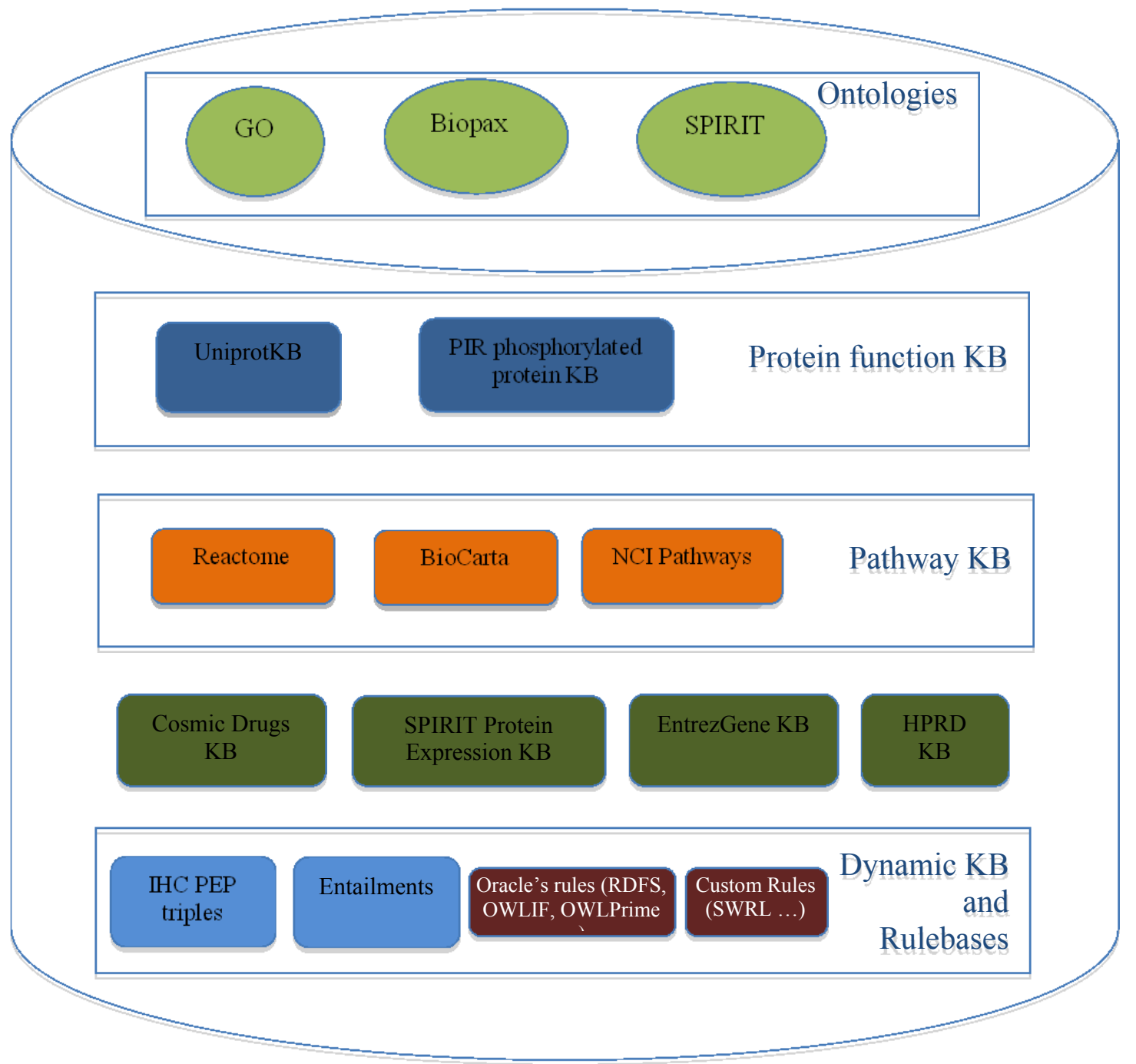

Figure 16 Semantic Web “Mashup” Knowledgbase (SWMKB) 
We investigated data integration issues related to the creation of SWMKB, which serves as the main source of genomic, proteomic and drug related data for building morphoproteomic diagrams. Oracle 11g [99] Semantic Web framework was chosen as an underlying platform for the knowledge base. SWMKB is a part of our SPIRIT (Systems Pathology InfoRmatIcs Toolbox) framework, which unites our IHC antibody test selection tool and computational morphoproteomic module with a common user interface. The instance data from EntrezGene [32] ( 330,000 RDF triples), Reactome [38] ( 700,000 RDF triples), HPRD [100] ( 40,000 RDF triples), [85] UniprotKB $(3,300,000$ RDF triples), and NCI Nature Pathways [101] ( 240,000 RDF triples) have been imported into the Oracle's RDF store totaling to more than 4,600,000 RDF triples of information. We then constructed and ran an initial set of SPARQL queries to assess performance of the "mashup" knowledge base. The speed of the simple queries was in the order of tens of a second, but the execution time of some queries containing a SPARQL UNION statement were in the order of a few minutes and more. We have observed that more work on refining the structure of the semantic "mashup" has to be done to satisfy the performance requirements of the complex morphoproteomic queries.

We have developed a method that tackles the problem of integrating heterogeneous data from publicly available "omics" data sources such as Uniprot, EntrezGene, BioCarta, Reactome. The data that are not available in Semantic Web compatible format are converted into RDF representation using $\mathrm{N}$-ary relation modeling and custom ontologies.

The SWMKB consists of several KB-components imported from online repositories. The knowledge about protein functions is represented by the functional annotations from Uniprot and Protein Information Resource (PIR) [102]. The "low-level" pathway data are 
imported from Reactome. This information helps to answer questions about "low-level" protein interactions at the level of biochemical reactions. We draw "high-level" pathway information from Nature curated pathway database (from Pathway Interaction Database [101]) and from BioCarta pathway database [103]. These sources contain "canonic" pathways that are frequently used by researchers in life sciences. We use EntrezGene functional annotation data for information about gene functions and COSMIC database [104] for drug-protein interactions information. Since UniprotKB, EntreGene and other involved resources all have different identification systems, we use information from Human Protein Reference Database [100] to link identifiers from these databases.

To our knowledge, there is not any comprehensive public source for protein expression data across different tissue types and diseases. For the purpose of this work, we use the database of protein expression patterns across hematopoietic tumors from our previous work [23] (shown as SPIRIT Protein Expression KB component in Fig. 16), supplementing it with additional data from biomedical literature and WHO guidelines [54].

Two main ontologies that we will utilize are GO [33] and Biopax [40] as shown on the top portion of Figure 3-6. GO was designed to capture the relationships among biological entities on the gene level. Biopax is an ontology that provides standardized access to many Biopax-compatible pathways repositories, including BioCarta, PID, and Reactome. The SPARQL queries and inference rules in our semantic "mashup" use the syntax and semantics of Biopax but not individual pathway-components' ontologies. To cover the rest of the entity relationships in the system we developed custom ontology 
shown as SPIRIT ontology in Fig. 3-6. For example, SPIRIT ontology describes concepts and relationships for COSMIC protein-drug interactions data (Fig. 17).

The inference model in our system employs above mentioned ontologies as well as semantic rulebases. Simple inferences like, general-to-specific relationships (e.g. interaction-to-biochemical reaction) are realized through ontological hierarchical (subclass/sub-property) relationships. More complex inferences (e.g. transitive relations) are implemented via custom rules. We also use built-in Oracle 11g Semantic Framework rulebases such as RDFS and OWLPrime.

One of the challenges of creating RDF "mashup" is that some data are not available in RDF N3 format. We have developed a method for converting such data based on $N$-ary relations modeling. An example of this methodology for transforming tabular drugprotein data into RDF is shown in Fig. 17 below.

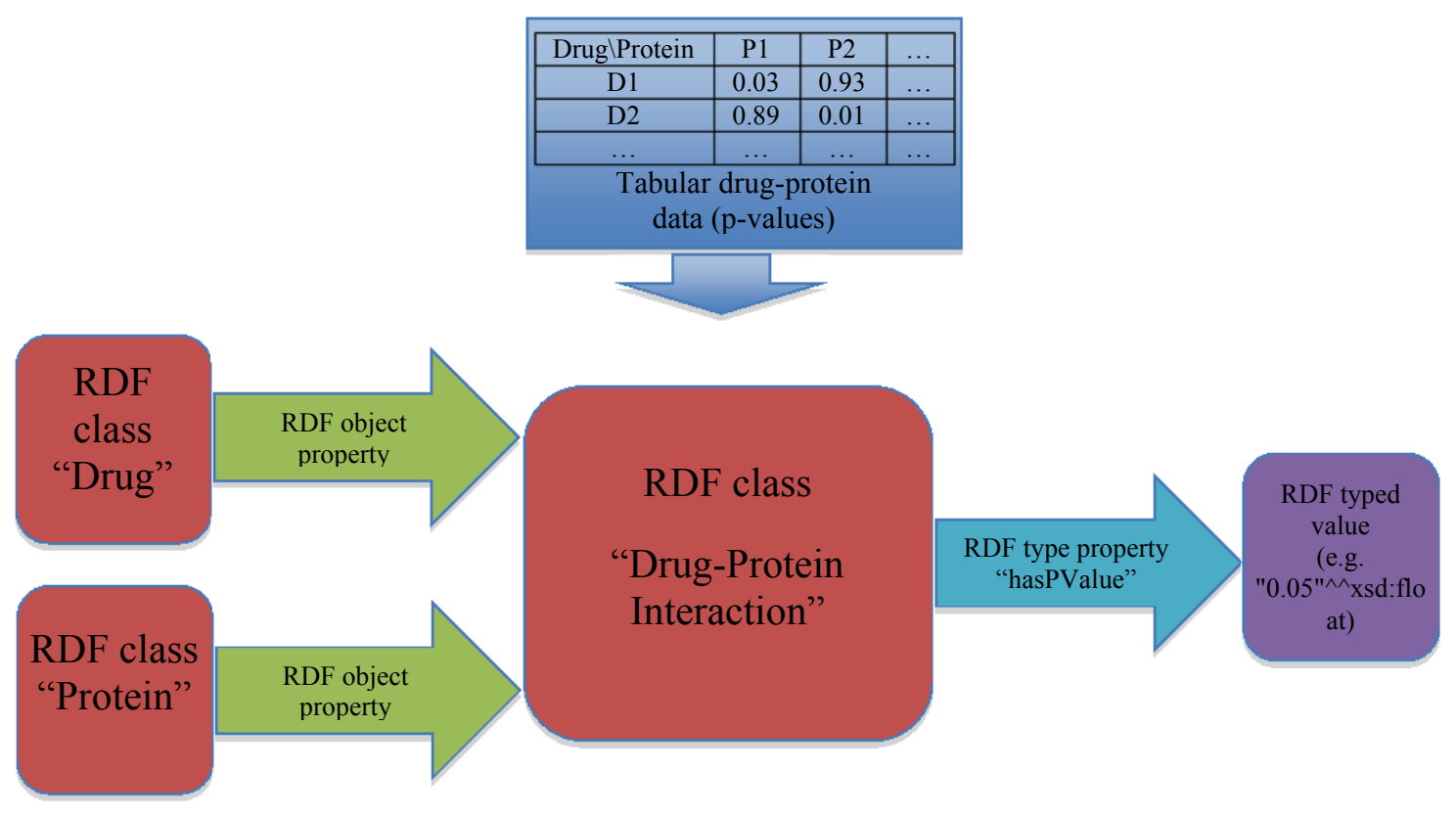

Figure 17 Conversion of $\mathrm{N}$-ary relations data into RDF 
Given drug-protein interactions' p-values in tabular format, each interaction will be modeled as a set of three RDF triples: (1) [Drug ID] [participates] [Drug-Protein Interaction ID], (2) [Protein ID] [participates] [Drug-Protein Interaction ID], and (3) [Drug-Protein Interaction ID] [hasPValue] [p-value] (p-value is shown as '"0.05"^^xsd:float' in the rightmost box in Fig.17). Higher dimensionality $\mathrm{N}$-ary relations will be represented similarly with "inter-entity" RDF class (e.g. "Drug-Protein Interaction" in the example above) having additional RDF type or object properties (see [25]).

\subsubsection{Inferring causal relationships from $R D F$ graphs}

To infer causal links for morphoproteomic studies from SWMKB we use the fact that this knowledgebase is actually a network. Since the data structure of SWMKB is based on RDF subject-predicate-object triples, where the object of each triple can in turn be the subject of another triple, the SWMKB forms a graph. We use Semantic Web SPARQL query language [105] to navigate this graph and to perform set operations needed for extraction of graphoids. The central part of the algorithm is a function that finds a path between two RDF nodes. For experiments in this dissertation, we extend an RDF path search function of the Jena [106] framework. The Jena's path search function implements a Breadth First Search (BFS) algorithm. However, it searches for possible paths between nodes only in the direction from subject to object. To understand that let us look at Fig. 18. 


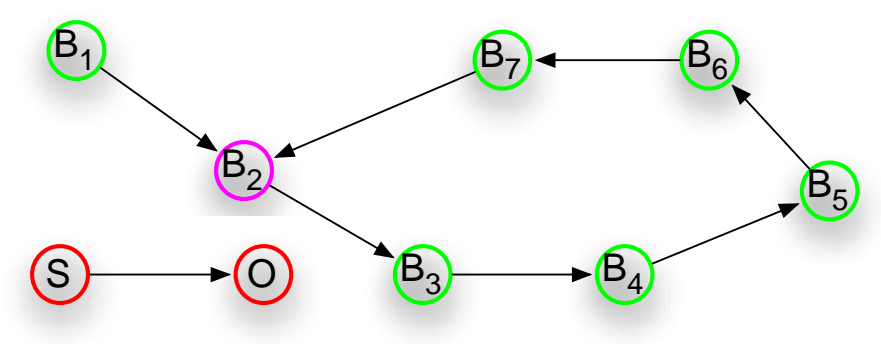

Figure 18 Multiple paths between two RDF nodes

In the RDF path $\mathrm{B}_{1}-\mathrm{B}_{2}-\mathrm{B}_{3}-\mathrm{B}_{4}-\mathrm{B}_{5}$, the object of each triple is the subject of the next adjacent triple. For example node $\mathrm{B}_{2}$ is the object of the triple $\mathrm{B}_{1}-\mathrm{B}_{2}$ and the subject of the triple $\mathrm{B}_{2}-\mathrm{B}_{3}$ and so on. Note that direction of the edges in Fig 18 neither corresponds to ontological directionality of predicates nor indicates causal relationships between RDF resources. It just merely serves to determine subject and object of the triple (the subject is adjacent to the tail of the edge and object to its arrowhead). The Jena's path search function was not designed to find paths where the object of a triple can serve as the object of adjacent triple (e.g. path $\mathrm{B}_{1}-\mathrm{B}_{2}-\mathrm{B}_{7}-\mathrm{B}_{6}-\mathrm{B}_{5}$ ). We extend this function to accommodate search for such paths. It however dramatically increases complexity of the search, which needs to be addressed in future research. In this dissertation we limit search space of the graph and demonstrate the utility of the proposed method on a subset of SWMKB.

To extract graphoids from the RDF graph of SWMKB we utilize a number of structure learning algorithms including PC (named after Peter Spirtes and Clark Glymour) [53] implemented in TETRAD package [107] and Inferred-Causation ICS [93] implemented in Weka [108]. In all cases we replace a function in these libraries that tests for conditional independence with our IsIndependent() function, the pseudo code of which is presented in Fig. 19. 


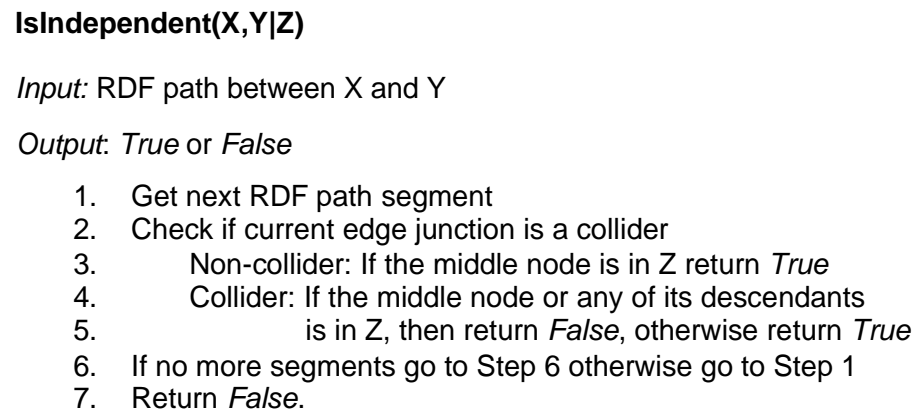

\section{Figure 19 Pseudo code of IsIndependent() function}

Different RDF nodes in the knowledge base can represent the same biological entity. When checking if a node $a$ is a member of the set $S$, we also need to check all other nodes that represent the same biological entity as node $a$ to see if any of them is in $S$. For instance, in Fig. 20 node $\mathrm{B}_{5}$ is not in $S$, but node $\mathrm{B}_{6}$ (let us call it alias of $\mathrm{B}_{5}$ ) that represents the same biological entity (through RDF predicate "XREF" in BioPax) is in S. We will, therefore, consider that node $\mathrm{B}_{5}$ is a member of $S$.

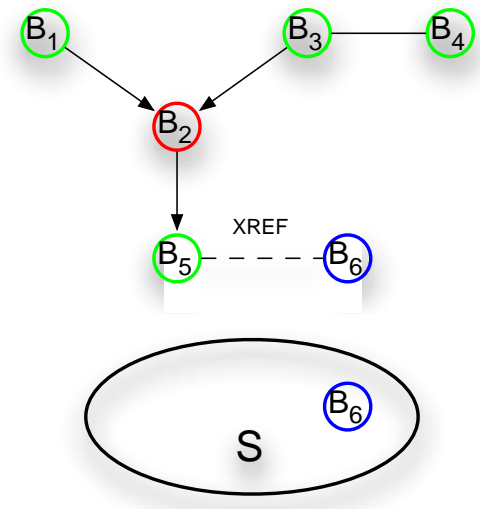

Figure 20 RDF graph without entailments

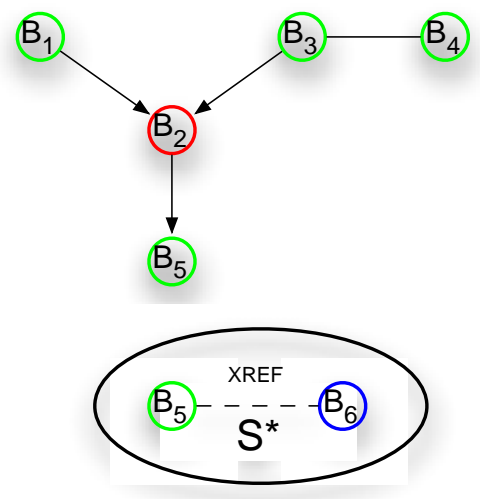

Figure 21 RDF graph with entailments 
To check if a node $a$ is a member of $S$ requires finding all aliases of node $a$. To reduce the execution time, we pre-compute entailments of the set $S$ and get an expanded set $S^{*}$. The entailments include all aliases of all nodes in $S$. We then check each RDF node against the expanded set $S^{*}$. Fig. 21 illustrates this process. The set $S^{*}$ includes node $\mathrm{B}_{5}$ as an entailment. To avoid finding all aliases of $\mathrm{B}_{5}$ during the query time, as shown in Fig. 20, we utilize a pre-computed set $S^{*}$ that will already have $\mathrm{B}_{5}$ as an alias of $\mathrm{B}_{6}$, which will lead to the same outcome in terms of conditional independency test but will execute much faster.

\subsection{Results and Discussion}

To assess the utility of the proposed approach for inferring casual links from RDF graphs of online knowledgebases, we have conducted three types of experiments. First, we have designed a "synthetic" model with predefined causal relationships and tested the validity of the algorithm to see if it can "recover" those relationships from an RDF representation of the model. Second, we have conducted validation experiments to see if our findings match results of morphoproteomic studies reported in the literature. And finally, we performed a comprehensive analysis that identifies additional causal links discovered by our algorithm in morphoproteomic studies. To do this a systematic literature review was conducted to support or reject new findings. The results of these experiments are presented in Sections 3.3.1, 3.3.2 and 3.3.3. In the end of Section 3 we discuss the benefits of our approach and implications of it for the field of computational morphoproteomics in particular and the biomedical domain in general. 


\subsubsection{Simulation Tests}

The main goal of the simulation experiments was to test whether the implementation of Conjecture 1 can correctly extract a set of predefined causal links. It must be said here that our simulation tests are not exhaustive, since the reversed process of constructing of a synthetic RDF graph for a set of predefined casual links may not be trivially performed for all cases.

In traditional probabilistic settings, information about causal relationships among entities of some physical process can be recovered from a probability distribution $P$ that captures frequencies of events associated with these entities. Simple probabilistic tests $P(X, Y \mid Z)=P(X \mid Z)$ can be used to extract graphoids $\mathrm{I}(\mathrm{X}, \mathrm{Y} \mid \mathrm{Z})$, which then can be used to extract causal relationships using constrained-based algorithms [53]. A synthetic dataset for a model with predefined causal links in this case can be constructed by simulating frequencies of data items that are consistent with the probabilistic tests and corresponding graphoids that are compatible with the predefined causal links.

In case with knowledgebases, we use Conjecture 1 to extract graphoids from RDF graphs of these knowledgebases. Therefore, to generate a synthetic RDF graph of a model with predefined causal links we need to make sure that all RDF graph components are constructed in such a way that after applying Conjecture 1 we can extract graphoids that are compatible with the original set of causal links. It has to be noted that there could be several different RDF graphs that are consistent with the same set of causal links. In other words, a set of causal links is not RDF-graph-isomorphic. 
For the purpose of simulation tests we defined a simple model of protein and pathway entities with two causal links that are modeled using a directional RDF predicate: (a) a protein $\mathrm{B}_{1}$ causes an activation of a pathway $\mathrm{B}_{5}$, and (b) a protein $\mathrm{B}_{4}$ causes an activation of the pathway $\mathrm{B}_{5}$. A possible RDF graph that is consistent with these links is shown in Fig. 22.

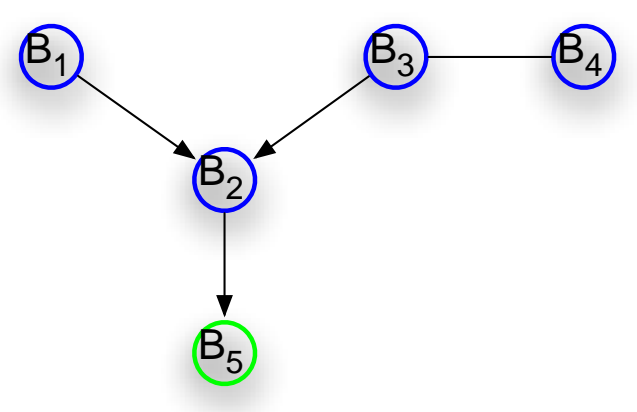

Figure 22 A “synthetic” RDF graph

To model causal links (a) and (b) we notice that both proteins $\mathrm{B}_{1}$ and $\mathrm{B}_{4}$ are causes of activation of the same pathway $\mathrm{B}_{5}$. In other words, the pathway $\mathrm{B}_{5}$ is a common effect for proteins $\mathrm{B}_{1}$ and $\mathrm{B}_{4}$. We could therefore use the explaining away technique [52] and model these causal links using a collider. We added protein nodes $\mathrm{B}_{2}$ and $\mathrm{B}_{3}$ to bring some complexity to the RDF graph without altering relationship among entities $\mathrm{B}_{1}, \mathrm{~B}_{4}$ and $\mathrm{B}_{5}$. The reason behind this addition was to check the correctness of the algorithm because proteins $B_{2}$ and $B_{3}$ should not change conditional independence of entities $B_{1}, B_{4}$, and $\mathrm{B}_{5}$ according to Conjecture 1. The actual collider here is an edge junction $\mathrm{B}_{1} \rightarrow \mathrm{B}_{2} \leftarrow \mathrm{B}_{3}$, but since $\mathrm{B}_{2}$ is the cause of $\mathrm{B}_{5}$, and $\mathrm{B}_{3}$ is "correlated" with $\mathrm{B}_{4}$, we should expect the extraction of the original causal links using Conjecture 1. The corresponding graphoids are $I\left(\mathrm{~B}_{1}, \mathrm{~B}_{3} \mid \mathrm{B}_{4}\right)$ because of Conjecture 1 Part $2, \mathrm{I}\left(\mathrm{B}_{1}, \mathrm{~B}_{4} \mid \mathrm{B}_{3}\right)$ because of 
Conjecture 1 Parts 1 and 2, and $\mathrm{I}\left(\mathrm{B}_{1}, \mathrm{~B}_{5} \mid \mathrm{B}_{2}\right), \mathrm{I}\left(\mathrm{B}_{4}, \mathrm{~B}_{5} \mid \mathrm{B}_{2}\right), \mathrm{I}\left(\mathrm{B}_{4}, \mathrm{~B}_{5} \mid \mathrm{B}_{3}\right), \mathrm{I}\left(\mathrm{B}_{3}, \mathrm{~B}_{5} \mid \mathrm{B}_{2}\right)$, $\mathrm{I}\left(\mathrm{B}_{4}, \mathrm{~B}_{2} \mid \mathrm{B}_{3}\right)$ because of Conjecture 1 Part 1.

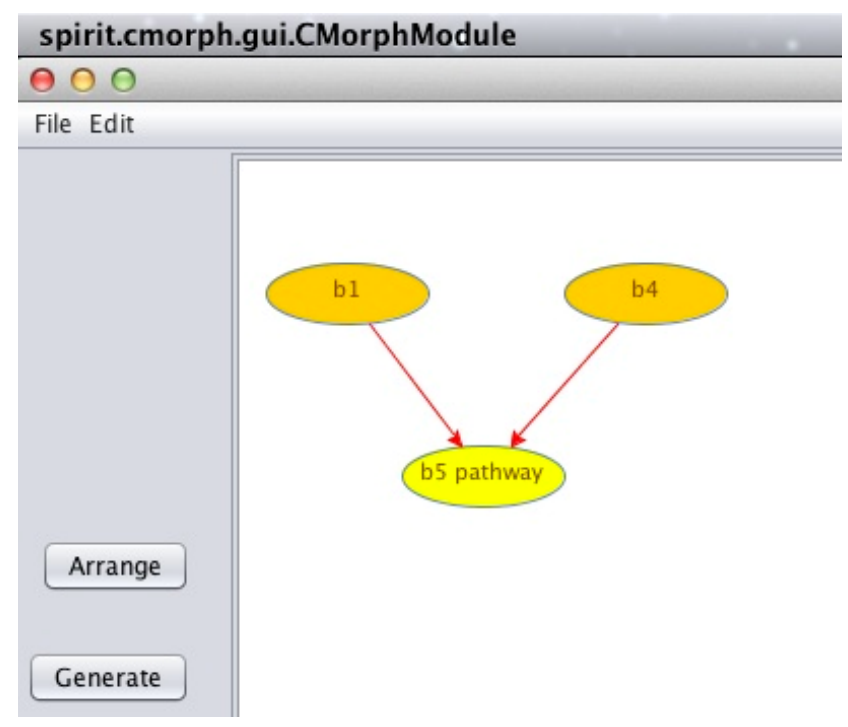

Figure 23 Recovered causal links from "synthetic” RDF graph

After running our algorithm for RDF graph shown in Fig. 22 for proteins $B_{1}$ and $B_{4}$ and pathway $\mathrm{B}_{5}$, correct causal links are obtained (Fig. 23).

\subsubsection{Validation of Morphoproteomic Studies}

We have run experiments with the protein and pathways from two morphoproteomic cases reported by the group of pathologists in [2] and [20].

In the first case, the pathologists used morphoproteomics to find alternate drug targets for a patient who did not respond to a conventional chemotherapy. They wanted to use a Bortezomib drug, a proteasome inhibitor, in a combination with another drug Dexamethasone, to treat relapsed Acute Lymphoblastic Leukemia (ALL). The goal was to construct a morphoproteomic diagram related to the case's biochemical processes in such 
a way that appropriate drug target can be identified. After conducting immunohistochemical (IHC) tests for paraffin-embedded tissue, correlative expression of $A K T, N F-$ kappa-B and BCL-2 proteins was revealed. The task then was to find biological circuitries that were consistent with the expression of these proteins. After analysis of several signal transduction pathways, the pathologists [2] came to the conclusion that an anti-apoptotic mechanism had been activated in this case, which led to the disruption of programmed cell death and therefore abnormal cell proliferation. More specifically, the activation of AKT pathway led to the activation of the of NF-kappa-B protein that in turn resulted in up-regulation of BCL-2 protein, which is known to be an anti-apoptotic agent.

To test our approach, we ran the algorithm for BCL-2, AKT and NF-kappa-B proteins and a set of signal transduction pathways that included AKT and NF-kappa-B pathways. The resulted diagram of extracted causal links is presented on Fig. 24.

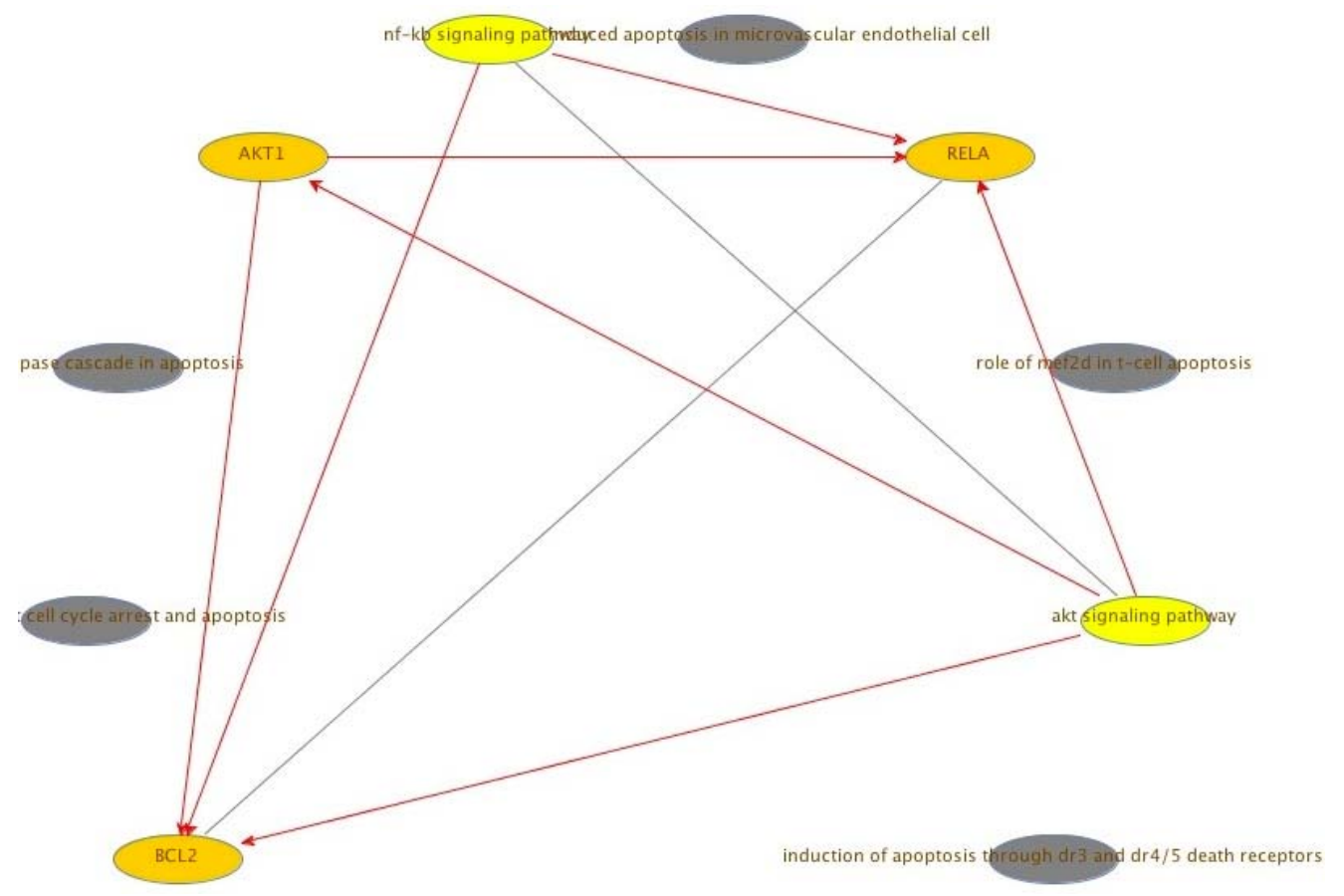

Figure 24 Uncovered morphoproteomic relationships Case 1 
As it can be seen from Fig. 24, the algorithm has identified the AKT pathway as the cause of the expression of RELA protein, which is a subunit of NF-kappa-B protein. Our method has also correctly positioned BCL-2 downstream from NF-kappa-B signaling pathway, which explains the expression of its main effector RELA (NF-kappa-B) protein.

The second morphoproteomic case [20] involved a study of Rapamycin drug as a potential treatment for Mesenchymal Chondrosarcoma. In this case IHC studies revealed cytoplasmic expression of phosphorylated AKT (p-AKT) and mTOR (p-mTOR) proteins as well as nuclear expression of phosphorylated p70 S6 kinase (p-p70S6K). After morphoproteomic analysis the conclusion was that $\mathrm{p}$-AKT causes an activation of $\mathrm{p}$ mTOR, which in turn phosphorylates p-p70S6K. Since mTOR is a molecular target of Rapamycin, it was suggested this drug be used to inhibit mTOR activity and therefore disrupt activation of p70S6K and consequent G1 cell cycle progression in malignant Mesenchymal Chondroblasts.

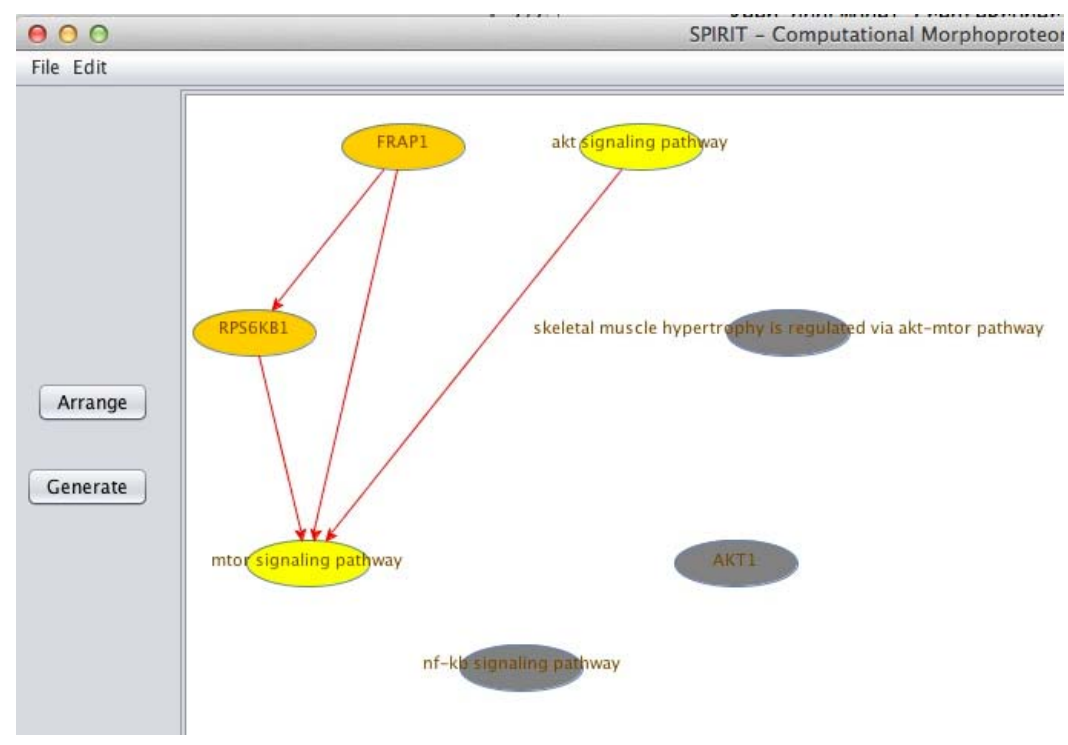

Figure 25 Uncovered morphoproteomic relationships Case 2 
To see if our method can produce the same morphoproteomic portrait, we ran the algorithm for AKT1, FRAP1 (alias for mTOR) and RPS6KB1(alias for $\mathrm{p} 70 \mathrm{~S} 6 \mathrm{~K}$ ) proteins and AKT and mTOR pathways. The morphoproteomic diagram with discovered causal links is presented in Fig. 25.

AKT is protein is known to be a key modulator of AKT pathway whose activity is closely related to mTOR pathway [85]. This fact is shown by a causal link on our diagram in Fig. 25. The second matched molecular mechanism is represented by a causal link from FRAP1 to RPS6KB1 proteins. It was reported in the literature that mTOR signaling pathway regulates FRAP1 (mTOR) protein kinase [109], which makes these two entities "interchangeable" in terms of the overall workflow of involved proteins and pathways. This demonstrates that revealed by our approach relationships confirm the findings described in [20].

The conducted experiments resulted in discovering causal links that match conclusions done by the pathologists. But unlike manual analysis by a human, these discoveries were performed by a computer, based on the information from the knowledgebase.

\subsubsection{New Findings in Morphoproteomic Studies}

Knowledge exploration through SPIRIT may effectively lead to the discovery of clinically useful information in regard to treatment of disease of all forms. In this dissertation, we focus on applications that identify new or novel means of treating malignancies that are advanced and resistant to the usual therapeutic modalities. 
Generally, these patients have already undergone treatment with multiple standard drug regimens and perhaps even experimental drug trials. It is in such cases that morphoproteomics provides essential information for personalized medical care based on the interpretation of findings in the biopsies of the patients own tumors in contrast to basing therapy on that general class of tumors. However, the linkage of visually observable tumor features to potentially effective chemotherapy requires a deep, as well as broad, knowledge of both cancer genomics and pharmacogenomics, which is well beyond the capability of most pathologists. Nonetheless, when provided with access to an extensive genomics database and a rational approach to information retrieval based on cellular pathways and protein interactions, a pathologist or oncologist can leverage his/her basic knowledge of disease to obtain more specific and relevant data through their own iterative interactions with the knowledge base.

For example, we consider the treatment of squamous cell carcinoma (SCC) which is a very common malignancy affecting an extensive variety of tissues. Feng et al. [11] have described their experience in marshalling proteomic evidence for the unconventional treatment of cervical squamous cell carcinoma by identifying over-expression of proteins within the epidermal growth factor receptor (EGFR) and the mammalian target of rapamycin (mTOR) signaling pathways. These pathways were evaluated in view of their known frequent involvement in malignancies as well as their susceptibility to pharmacologic inhibition. The EGF pathway may be down regulated with erlotinib and mTOR with an analogue of rapamycin, temsirolimus. The authors conclude that such a regimen would be reasonable for the treatment of early stage cervical SCC. 
In order to investigate the potential for SPIRIT to contribute new knowledge relevant to the treatment of SCC in general, we queried the knowledge base for other possible protein interactions in the EGFR signaling pathway that were not investigated by Feng et al. [11].

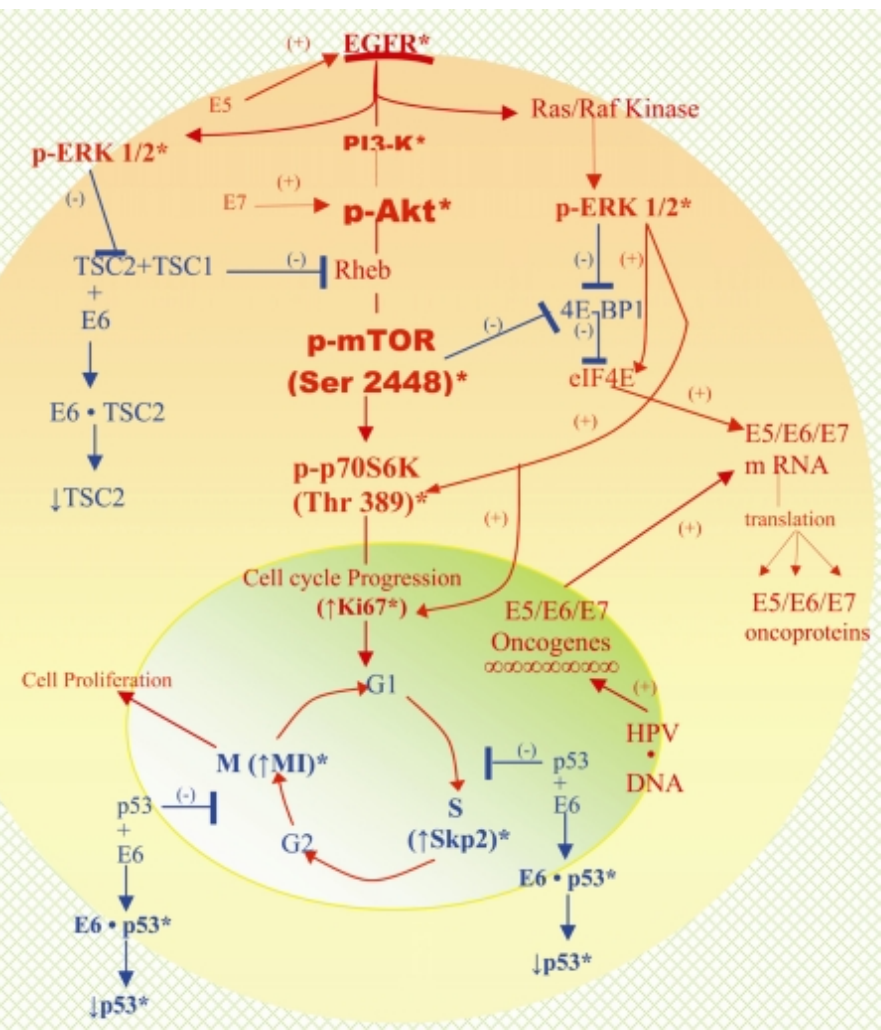

Figure 26 Morphoproteomic diagram from Feng et al. (included with permission from R. Brown and Int J Clin Exp Pathol)

Starting with a simplified schematic illustration of the EGFR pathway in Fig. 26, several proteins were queried leading to the identification of interactions between JAK1, RASA1, STAT1 and STAT3 (Signal transducers and activators of transcription 3) which are components of the JAK-STAT signaling pathway. To check protein and pathway 
relationships discovered by our method a systematic literature review was conducted using a set of queries for PubMed searches.

STAT1 and STAT3 do indeed interact. The following query returned 191 articles that support activation of STAT1 by STAT3, with [110], [111] being the representative papers.

<stat1[All Fields] AND ("stat3 transcription factor"[MeSH Terms] OR ("stat3"[All Fields] AND "transcription"[All Fields] AND "factor"[All Fields]) OR "stat3 transcription factor"[All Fields] OR "stat3"[All Fields]) AND ("Interaction"[Journal] OR "interaction"[All Fields])>

$\underline{\text { STAT3 over-expression is an important factor in SCC acting as an oncogenic }}$ transcription factor, which regulates a number of pathways important in neoplastic transformation including angiogenesis, cell cycle regulation and apoptosis [7], [112-118]. The following query returned 87 articles supporting the involvement of STST3 in SCC.

<carcinoma, squamous cell"[MeSH Terms] OR ("carcinoma"[All Fields] AND "squamous"[All Fields] AND "cell"[All Fields]) OR "squamous cell carcinoma"[All Fields] OR ("squamous"[All Fields] AND "cell"[All Fields] AND "carcinoma"[All Fields])) AND ("stat3 transcription factor"[MeSH Terms] OR ("stat3"[All Fields] AND "transcription"[All Fields] AND "factor"[All Fields]) OR "stat3 transcription factor"[All Fields] OR "stat3"[All Fields])>

STAT3 may be down regulated by a wide range of compounds including clinically utilized small molecule inhibitors, numerous experimental drugs, small interfering RNAs, and nutrigenic substances including the green tea extract, EGCG [111], [119-123]. The 
systematic literature review using the following query returned 2059 supporting the inhibition of STAT3.

$<($ "inhibition (psychology)"[MeSH Terms] OR ("inhibition"[All Fields] AND "(psychology)"[All Fields]) OR "inhibition (psychology)"[All Fields] OR "inhibition"[All Fields]) AND ("stat3 transcription factor"[MeSH Terms] OR ("stat3"[All Fields] AND "transcription"[All Fields] AND "factor"[All Fields]) OR "stat3 transcription factor"[All Fields] OR "stat3"[All Fields])>

And finally, native and activated (phosphorylated) STAT3 proteins may be identified with routine immunohistochemical staining with localization in the cytoplasm and nucleus respectively. We executed the following query which returned 1067 supporting papers, with [113], [115], [117], [124-127] being representative.

$<$ ("stat3 transcription factor"[MeSH Terms] OR ("stat3"[All Fields] AND "transcription"[All Fields] AND "factor"[All Fields]) OR "stat3 transcription factor"[All Fields] OR "stat3"[All Fields]) AND ("immunohistochemistry"[MeSH Terms] OR "immunohistochemistry"[All Fields])> 
The interactions that have been revealed by our approach are shown in Fig. 27.

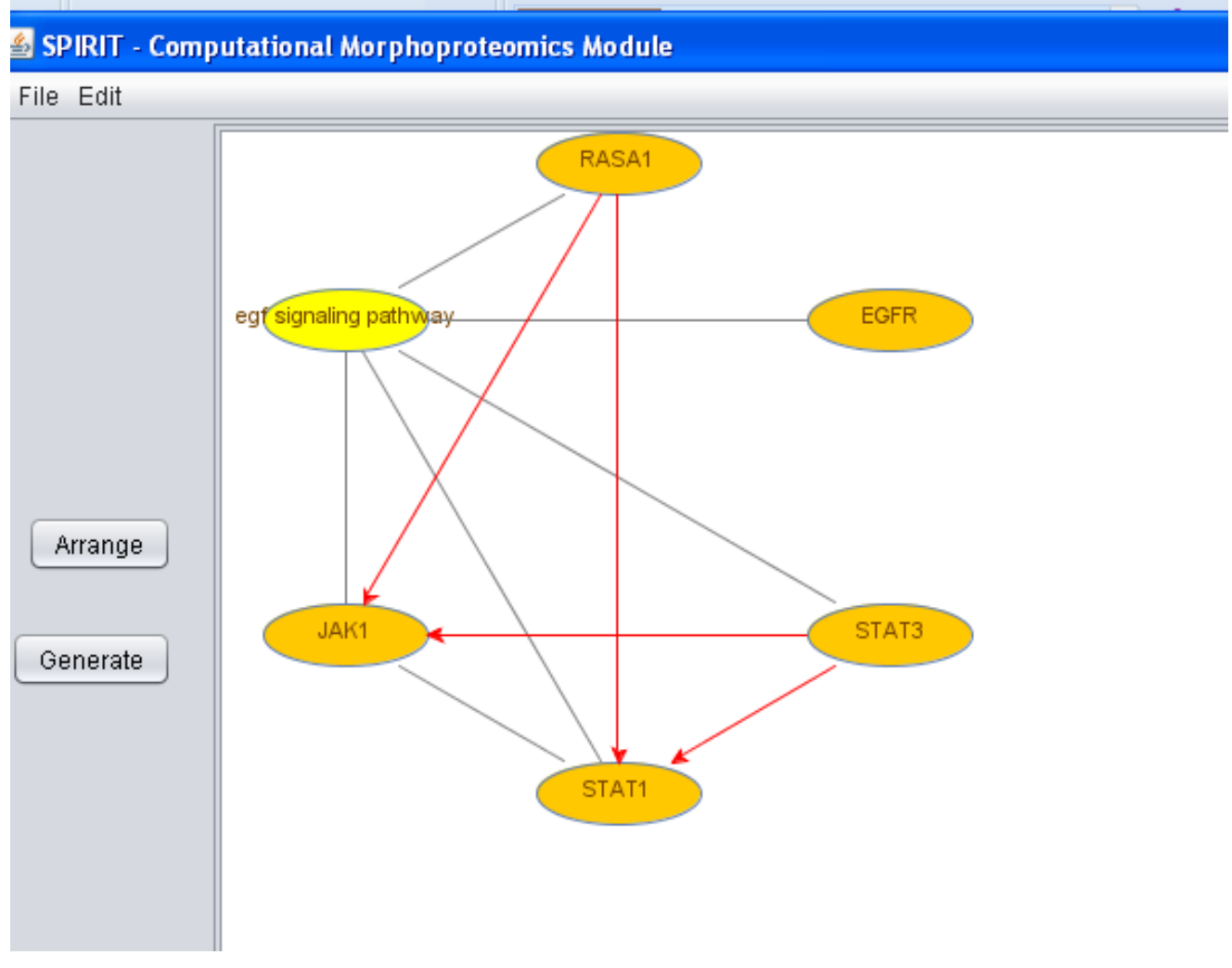

Figure 27 Uncovered morphoproteomic relationships Case 3

Therefore, this experiment demonstrates the type of expanded and newly discovered knowledge that is facilitated by SPIRIT. Some of the factual knowledge acquired through the assistance of SPIRIT may be summarized as follows: The activation and overexpression of the transcription factor STAT3 plays an important role in the SCC genomic apparatus. The activation of this protein may be identified by the presence of the phosphorylated form and its translocation from the cytoplasm to the nucleus. STAT3 expression has been demonstrated to be down regulated by several pharmacologic agents. These agents have shown some experimental effectiveness in other malignancies including breast carcinoma and chronic myeloid leukemia. 


\subsubsection{Discussion of Proposed Approach and Implications to Biomedical Domain}

We believe that our computational approach for discovering relationships among proteins and pathways will have broad impact on personalized medicine. Generated by our methods morphoproteomic diagrams will enable pathologists and oncologists to effectively utilize vast amounts of publicly available "omics" data to find new drug targets.

First of all, the central goal of the proposed method is to infer causal dependencies among biological entities based on data from public biomedical databases. Even though traditional bioinformatics research does involve studies of casual links in protein-protein interactions or biological pathways, we, however, found that the research community still needs significant work that focuses on inferring casual relationships from online knowledgebases. Unlike most of the current bioinformatics studies, which utilize either wet lab results or results from mining unstructured literature, our approach draws its conclusion from high quality, curated data from sources that are considered by the scientific community as the state-of-the-art knowledge of biochemical processes (e.g. Reactome, UniProt and EntrezGene). The result of a traditional bioinformatics experiment is greatly dependent on the conditions and type of wet lab study (e.g., study protocol, contamination, normalization method) that provides low-level "raw" data for computational processing. That explains possible heterogeneity of the results. In this research, we use high-level data that have been reviewed and accepted by the experts in the field and confirmed as a consensus for a number of wet lab experiments done by 
different investigators. Moreover, since we use data sources that include citations of original articles from which a certain fact of the knowledgebase was generated, we can provide links to those articles during morphoproteomic experiments to "justify" any conclusions that our framework might make.

Second of all, given the task of finding a mechanism that explains protein expression pattern from an IHC study, we can search a large number of signal transduction pathways and proteins. In [6] Brown mentions numerous collaborations with pathologists from other institutions. The primary goal for this is to use the knowledge of other experts to be able to check the pattern against a larger set of biochemical processes, to find a better place of intervention. In our case, the SPIRIT data integration methodology does not put a cap on the number of its constituent knowledgebases. In our opinion, SPIRIT has greater chance to provide more comprehensive molecular pathology picture of what is happening in diseased tissue that even an extended team of experienced pathologists.

Next, the underlying SPIRIT Semantic Web framework allows our method to use predicate logic and entailments to flexibly make connections among different entities in the "mashup". We utilize the Sematic Web rules and entailments to deduce new knowledge that is not presented in the $\mathrm{KB}$ directly. It provides means to solve, for instance, the problem with aliases. It is a known fact that in most cases biomedical databases list different names for the same entity. If a researcher wants to find an interaction using the name of a protein, he/or she may need to list all its aliases. We define Sematic Web rules to tie together RDF resources that represent the same biological entitles. Moreover, given a protein, we expect our approach to be able to associate it with other proteins that are either from a related functional family, it sub-units 
or from the same protein complex. That increases a chance of potentially discovering biochemical mechanisms that would otherwise be missed.

Our approach represents a computerized Pathologist-In-The-Loop setting which serves a hypothesis generator. This approach leverages the endurance of a computer to process vast amounts of data and the creative power of a human brain to make the final decision.

Finally, the proposed methods have great potential to be extended to other areas of biomedicine. For instance, SPIRIT can be used to generate hypotheses in the more general systems biology domain. We can alter the SWMKB's Semantic Web rules and add new ontologies with RDF data sources to tackle more general problems of interrelationships among proteins and pathways on a systems biology level. Theoretically, the proposed formalisms of inferring casual links from RDF knowledgebases can be utilized in any field of pathology, we just need to represent the data in RDF format and supply required ontologies. One of such areas could become a forensic pathology, where causeand-effect conclusions are sought in the basis of available facts and common knowledge of physiological and pathological processes.

\section{Summary and Future Work}

\subsection{Summary}

In this dissertation we have presented two novel informatic methods to help pathologists streamline and improve molecular pathology studies. 
The ATOS tool optimizes IHC antibody test selection by employing Shannon's entropy maximization idea to select the most informative antigen at each step. This results in a more than three-fold reduction of the turn-a-round time, but most importantly dramatically decreases inter- and intra-pathologist variability in IHC studies.

The second proposed method brings the theory of causation into the area of Semantic Web knowledgebases of protein and signal transduction pathways. We extend Pearl's formalism [52], [96] to infer causal links among proteins and pathways from RDF graphs of these knowledgebases. In our opinion, this method not only makes the new field of morphoproteomics more feasible to practice by pathologists and oncologists alike, but also has great potential to be extended into molecular systems biology and other biomedical domains.

Both tools are parts of our larger computational SPIRIT framework that is intended to facilitate contribution of pathology to personalized medicine and the new emerging field of theranostics.

\subsection{Future Work}

We have identified three directions for the future research related to the methods discussed in this dissertation. All three are concerned with improving our computational morphoproteomics module of the SPIRIT framework, i.e. the method of inferring causal links from RDF graphs. These future research directions are presented below. 
Currently, SPIRIT is designed to only depict a possible picture of causal morphoproteomic mechanisms and to facilitate generation of new hypotheses. However, the main morphoproteomic goal is to search for new effective drug targets, i.e., places in the web of inter-relationships among proteins and pathways that can be interrupted to prevent undesired physiological processes from happening. Therefore, an effective computational method to search for drug targets is needed. There are several issues associated with such a search. As one can see from Fig. 24, the AKT pathway is associated with more than one path that ends with BCL-2 protein. It is shown as a direct cause of expression of BCL-2, but can also be viewed as an indirect cause of this protein's activity through its constituent protein AKT1, RELA and NF-kappa-B pathway mechanism. In other words, our framework is set to discover any causal link between any two biological entities. We need a means to analyze such paths and select the ones that can serve as candidates for interruption by drugs. We also need to analyze drug targets on their clinical merits as well as their potential to cause undesired side effects.

Another challenge is related to the fact that the success of discovering causal links by constrained-based structure learning algorithms greatly depends on the sufficiency of supplied graphoids. We have noticed that adding an additional graphoid can "trigger" the algorithm to discover a causal link, which would otherwise be missed. We believe that this happens because of insufficiency of the set of constraints (graphoid) for the causal formalism to infer causality. Therefore, we need to extend the formalism to suggest possible graphoids, the addition of which can lead to a larger number of discovered causal links. 
More research is needed to improve the efficiency of RDF path computations. Since RDF paths are computed on the basis of SPARQL queries, we could explore parallel processing algorithms including those based on MapReduce and Hadoop technologies $[128,129]$. 


\section{BIBLIOGRAPHY}

[1] G. S. L. Center, "Challenges and Issues in Personalized Medicine and Pharmacogenetics.," Learn.Genetics, 2011. [Online]. Available: http://learn.genetics.utah.edu/content/health/pharma/issues/. [Accessed: 17Nov.-2011].

[2] R. E. Brown, B. Bostrom, and P. L. Zhang, "Morphoproteomics and bortezomib/dexamethasone-induced response in relapsed acute lymphoblastic leukemia," Ann Clin Lab Sci, vol. 34, no. 2, pp. 203-205, 2004.

[3] R. E. Brown, "Morphoproteomics: exposing protein circuitries in tumors to identify potential therapeutic targets in cancer patients," Expert Rev Proteomics, vol. 2, no. 3, pp. 337-348, Jun. 2005.

[4] J. Liu and R. E. Brown, "Morphoproteomics demonstrates activation of mTOR pathway in anaplastic thyroid carcinoma: a preliminary observation," Ann Clin Lab Sci, vol. 40, no. 3, pp. 211-217, 2010.

[5] J. Liu and R. E. Brown, "Morphoproteomics demonstrates activation of mammalian target of rapamycin pathway in papillary thyroid carcinomas with nuclear translocation of MTOR in aggressive histological variants," Aug. 2011.

[6] R. E. Brown, "Morphogenomics and morphoproteomics: a role for anatomic pathology in personalized medicine," Arch Pathol Lab Med, vol. 133, no. 4, pp. 568-579, Apr. 2009.

[7] V. Subbiah, A. Naing, R. E. Brown, H. Chen, L. Doyle, P. LoRusso, R. Benjamin, P. Anderson, and R. Kurzrock, "Targeted morphoproteomic profiling of Ewing's sarcoma treated with insulin-like growth factor 1 receptor (IGF1R) inhibitors: response/resistance signatures," PloS one, vol. 6, no. 4, p. e18424, 2011.

[8] S. Dhingra, M. E. Rodriguez, Q. Shen, X. Duan, M. L. Stanton, L. Chen, R. Zhang, and R. E. Brown, "Constitutive activation with overexpression of the mTORC2-phospholipase D1 pathway in uterine leiomyosarcoma and STUMP: morphoproteomic analysis with therapeutic implications," International journal of clinical and experimental pathology, vol. 4, no. 2, pp. 134-146, 2011.

[9] Q. Shen, M. L. Stanton, W. Feng, M. E. Rodriguez, L. Ramondetta, L. Chen, R. E. Brown, and X. Duan, "Morphoproteomic analysis reveals an overexpressed and constitutively activated phospholipase D1-mTORC2 pathway in endometrial carcinoma," International journal of clinical and experimental pathology, vol. 4, no. 1, pp. 13-21, 2010.

[10] M. J. Zenali, P. L. Zhang, A. E. Bendel, and R. E. Brown, "Morphoproteomic confirmation of constitutively activated mTOR, ERK, and NF-kappaB pathways in Ewing family of tumors," Ann Clin Lab Sci, vol. 39, no. 2, pp. 160-166, 2009.

[11] W. Feng, X. Duan, J. Liu, J. Xiao, and R. E. Brown, "Morphoproteomic evidence of constitutively activated and overexpressed mTOR pathway in 
cervical squamous carcinoma and high grade squamous intraepithelial lesions," International journal of clinical and experimental pathology, vol. 2, no. 3, pp. 249-260, 2009.

[12] R. E. Brown, G. Zotalis, P. L. Zhang, and B. Zhao, "Morphoproteomic confirmation of a constitutively activated mTOR pathway in high grade prostatic intraepithelial neoplasia and prostate cancer," International journal of clinical and experimental pathology, vol. 1, no. 4, pp. 333-342, 2008.

[13] W. Feng, R. E. Brown, C. D. Trung, W. Li, L. Wang, T. Khoury, S. Alrawi, J. Yao, K. Xia, and D. Tan, "Morphoproteomic profile of mTOR, Ras/Raf kinase/ERK, and NF-kappaB pathways in human gastric adenocarcinoma," Ann Clin Lab Sci, vol. 38, no. 3, pp. 195-209, 2008.

[14] R. E. Brown, D. Tan, J. S. Taylor, M. Miller, J. W. Prichard, and M. M. Kott, "Morphoproteomic confirmation of constitutively activated mTOR, ERK, and NF-kappaB pathways in high risk neuro-blastoma, with cell cycle and protein analyte correlates," Ann Clin Lab Sci, vol. 37, no. 2, pp. 141-147, 2007.

[15] P. L. Zhang, A. T. J. Quiery, T. M. Blasick, and R. E. Brown, "Morphoproteomic expression of H-ras (p21ras) correlates with serum monoclonal immunoglobulin reduction in multiple myeloma patients following pamidronate treatment," Ann Clin Lab Sci, vol. 37, no. 1, pp. 3438, 2007.

[16] R. E. Brown and A. Law, "Morphoproteomic demonstration of constitutive nuclear factor-kappaB activation in glioblastoma multiforme with genomic correlates and therapeutic implications," Ann Clin Lab Sci, vol. 36, no. 4, pp. 421-426, 2006.

[17] F. Lin, P. L. Zhang, X. J. Yang, J. W. Prichard, M. Lun, and R. E. Brown, "Morphoproteomic and molecular concomitants of an overexpressed and activated mTOR pathway in renal cell carcinomas," Ann Clin Lab Sci, vol. 36, no. 3, pp. 283-293, 2006.

[18] R. E. Brown, P. L. Zhang, M. Lun, S. Zhu, P. K. Pellitteri, W. Riefkohl, A. Law, G. C. Wood, and T. L. Kennedy, "Morphoproteomic and pharmacoproteomic rationale for mTOR effectors as therapeutic targets in head and neck squamous cell carcinoma," Ann Clin Lab Sci, vol. 36, no. 3, pp. 273-282, 2006.

[19] R. E. Brown, "Brief communication: morphoproteomic analysis of osteolytic Langerhans cell histiocytosis with therapeutic implications," Ann Clin Lab Sci, vol. 35, no. 2, pp. 131-136, 2005.

[20] R. E. Brown, "Morphoproteomic portrait of the mTOR pathway in mesenchymal chondrosarcoma," Ann Clin Lab Sci, vol. 34, no. 4, pp. 397399, 2004.

[21] R. E. Brown, M. Lun, J. W. Prichard, T. M. Blasick, and P. L. Zhang, "Morphoproteomic and pharmacoproteomic correlates in hormone-receptornegative breast carcinoma cell lines," Ann Clin Lab Sci, vol. 34, no. 3, pp. 251-262, 2004.

[22] M. Lun, P. L. Zhang, N. Siegelmann-Danieli, T. M. Blasick, and R. E. Brown, "Intracellular inhibitory effects of Velcade correlate with 
morphoproteomic expression of phosphorylated-nuclear factor-kappaB and p53 in breast cancer cell lines," Ann Clin Lab Sci, vol. 35, no. 1, pp. 15-24, 2005.

[23] D. Shin, G. Arthur, and C.-R. Shyu, “An Entropy Based Probabilistic Framework to Optimize Immunohistochemical Studies in Digital Pathology Setting," presented at the American Medical Informatics Association, Washington DC, USA, 2010, p. 1255.

[24] D. Shin, G. Arthur, C. Caldwell, M. Popescu, M. Petruc, A. Diaz-Arias, and C. Shyu, "A pathologist-in-the-loop IHC antibody test selection using the entropy-based probabilistic method," Journal of pathology informatics, vol. 3, no. 1, p. 1, 2012.

[25] S. Warner, "Diagnostics + therapy $=$ theranostics: strategy requires teamwork, partnering, and tricky regulatory maneuvering," The Scientist, Aug. 2004.

[26] W3C, "Semantic Web," w3.org. [Online]. Available: http://www.w3.org/standards/semanticweb/. [Accessed: 24-Jun.-2012]. immunohistochemistry in the diagnosis of non-Hodgkin and Hodgkin lymphoma," Arch Pathol Lab Med, vol. 132, no. 3, pp. 441-461, Mar. 2008. M. D. Feldman, "Beyond morphology: whole slide imaging, computer-aided detection, and other techniques," Arch Pathol Lab Med, vol. 132, no. 5, pp. 758-763, May 2008.

S. S. Sahoo, K. Zeng, O. Bodenreider, and A. Sheth, "From "glycosyltransferase" to 'congenital muscular dystrophy': integrating knowledge from NCBI Entrez Gene and the Gene Ontology," Stud Health

Technol Inform, vol. 129, no. 2, pp. 1260-1264, 2007.
S. S. Sahoo, O. Bodenreider, J. L. Rutter, K. J. Skinner, and A. P. Sheth, “An ontology-driven semantic mashup of gene and biological pathway information: application to the domain of nicotine dependence," $J$ Biomed Inform, vol. 41, no. 5, pp. 752-765, Oct. 2008.

C. J. Vaske, S. C. Benz, J. Z. Sanborn, D. Earl, C. Szeto, J. Zhu, D. Haussler, and J. M. Stuart, "Inference of patient-specific pathway activities from multidimensional cancer genomics data using PARADIGM.," Bioinformatics, vol. 26, no. 12, pp. i237-45, Jun. 2010.

D. Maglott, J. Ostell, K. D. Pruitt, and T. Tatusova, "Entrez Gene: genecentered information at NCBI," Nucleic Acids Res, vol. 33, no. Database issue, 2005.

M. Ashburner, C. A. Ball, J. A. Blake, D. Botstein, H. Butler, J. M. Cherry, A. P. Davis, K. Dolinski, S. S. Dwight, J. T. Eppig, M. A. Harris, D. P. Hill, L. Issel-Tarver, A. Kasarskis, S. Lewis, J. C. Matese, J. E. Richardson, M. Ringwald, G. M. Rubin, and G. Sherlock, "Gene ontology: tool for the unification of biology. $\{\mathrm{T}\}$ he $\{\mathrm{G}\}$ ene $\{\mathrm{O}\}$ ntology $\{\mathrm{C}\}$ onsortium," Nature Genetics, vol. 25, no. 1, pp. 25-29, 2000.

"Online Mendelian Inheritance in Man, OMIM (TM)," ncbi.nlm.nih.gov. [Online]. Available: http://www.ncbi.nlm.nih.gov/omim/. [Accessed: 24Jun.-2012]. 
D. McGuinness and F. van Harmelen, "OWL Web Ontology Language Overview," 2004. E. Prud'hommeaux and A. Seaborne, "SPARQL Query Language for RDF," 2008. M. Kanehisa and S. Goto, "KEGG: Kyoto Encyclopedia of Genes and Genomes," Nucleic Acids Res, vol. 28, no. 1, pp. 27-30, 2000.

D. Croft, G. O'Kelly, G. Wu, R. Haw, M. Gillespie, L. Matthews, M. Caudy, P. Garapati, G. Gopinath, B. Jassal, S. Jupe, I. Kalatskaya, S. Mahajan, B. May, N. Ndegwa, E. Schmidt, V. Shamovsky, C. Yung, E. Birney, H. Hermjakob, P. D'Eustachio, and L. Stein, "Reactome: a database of reactions, pathways and biological processes," Nucleic Acids Res, vol. 39, no. Database issue, pp. D691-D697, 2011.

P. Karp, C. Ouzounis, C. Moore-Kochlacs, L. Goldovsky, P. Kaipa, D. Ahrén, S. Tsoka, N. Darzentas, V. Kunin, and N. López-Bigas, "Expansion of the BioCyc collection of pathway/genome databases to 160 genomes," Nucleic Acids Res, vol. 33, no. 19, pp. 6083-6089, 2005.

E. Demir, M. P. Cary, S. Paley, K. Fukuda, C. Lemer, I. Vastrik, G. Wu, P. D'Eustachio, C. Schaefer, J. Luciano, F. Schacherer, I. Martinez-Flores, Z. $\mathrm{Hu}$, V. Jimenez-Jacinto, G. Joshi-Tope, K. Kandasamy, A. C. Lopez-Fuentes, H. Mi, E. Pichler, I. Rodchenkov, A. Splendiani, S. Tkachev, J. Zucker, G. Gopinath, H. Rajasimha, R. Ramakrishnan, I. Shah, M. Syed, N. Anwar, O. Babur, M. Blinov, E. Brauner, D. Corwin, S. Donaldson, F. Gibbons, R. Goldberg, P. Hornbeck, A. Luna, P. Murray-Rust, E. Neumann, O. Ruebenacker, O. Reubenacker, M. Samwald, M. van Iersel, S. Wimalaratne, K. Allen, B. Braun, M. Whirl-Carrillo, K.-H. Cheung, K. Dahlquist, A. Finney, M. Gillespie, E. Glass, L. Gong, R. Haw, M. Honig, O. Hubaut, D. Kane, S. Krupa, M. Kutmon, J. Leonard, D. Marks, D. Merberg, V. Petri, A. Pico, D. Ravenscroft, L. Ren, N. Shah, M. Sunshine, R. Tang, R. Whaley, S. Letovksy, K. H. Buetow, A. Rzhetsky, V. Schachter, B. S. Sobral, U. Dogrusoz, S. McWeeney, M. Aladjem, E. Birney, J. Collado-Vides, S. Goto, M. Hucka, N. Le Novère, N. Maltsev, A. Pandey, P. Thomas, E. Wingender, P. D. Karp, C. Sander, and G. D. Bader, "The BioPAX community standard for pathway data sharing.," Nat. Biotechnol., vol. 28, no. 9, pp. 935-942, Sep. 2010.

S. A. Kauffman, "Metabolic stability and epigenesis in randomly constructed genetic nets.," J Theor Biol, vol. 22, no. 3, pp. 437-467, Mar. 1969.

S. S. Bornholdt, "Boolean network models of cellular regulation: prospects and limitations.," J R Soc Interface, vol. 5, pp. S85-S94, Aug. 2008.

S. Liang, S. Fuhrman, and R. Somogyi, "Reveal, a general reverse engineering algorithm for inference of genetic network architectures," Pacific symposium on ..., 1998. I. Shmulevich, E. R. Dougherty, and S. F. I. A. A. Mathematics, Probabilistic Boolean Networks. Siam, 2009, p. 267. T. Schlitt and A. Brazma, "Current approaches to gene regulatory network modelling.," BMC Bioinformatics, vol. 8, p. S9, 2007.

M. Hecker, S. Lambeck, S. Toepfer, E. van Someren, and R. Guthke, "Gene 
regulatory network inference: data integration in dynamic models-a review.," BioSystems, vol. 96, no. 1, pp. 86-103, Apr. 2009.

[47] D. Koller and N. Friedman, Probabilistic Graphical Models: Principles (text only) by D.Koller.N.Friedman. The MIT Press, 2009, p. 1231.

M. I. Jordan, Learning in Graphical Models. The MIT Press, 1999.

D. Edwards, Introduction to Graphical Modelling, 2nd ed. Springer, 2000, p. 333.

[50] S. L. Lauritzen, Graphical Models. Oxford University Press, USA, 1996, p. 298.

[51] C. Borgelt, M. Steinbrecher, and R. Kruse, Graphical Models. Wiley, 2009, p. 404.

[52] J. Pearl, Probabilistic Reasoning in Intelligent Systems: Networks of Plausible Inference, 1st ed. Morgan Kaufmann, 1988, p. 552.

[53] C. Spirtes, P. Glymour, and R. Scheines, Causation, Prediction and Search. The MIT Press, 2001, p. 964.

J. Whittaker, Graphical Models in Applied Multivariate Statistics, 1st ed. Wiley, 2009, p. 462.

S. G. Brush, "History of the Lenz-Ising Model," Reviews of Modern Physics, vol. 39, no. 4, pp. 883-893, Oct. 1967.

A. Freno and E. Trentin, Hybrid Random Fields. Springer Verlag, 2011, p. 210.

A. Freno, E. Trentin, and M. Gori, "A hybrid random field model for scalable statistical learning.," Neural networks : the official journal of the International Neural Network Society, vol. 22, no. 5, pp. 603-613, Jul. 2009. Y. Zhou, "Structure Learning of Probabilistic Graphical Models: A Comprehensive Survey," CORD Conference Proceedings, pp. -, Nov. 2011. J. Rissanen, Stochastic complexity in statistical inquiry. World Scientific Pub Co Inc, 1989, p. 177.

G. F. Cooper and E. Herskovits, A Bayesian method for the induction of probabilistic networks from data. 1991, p. 41.

D. Heckerman, D. Geiger, and D. M. Chickering, "Learning Bayesian networks: The combination of knowledge and statistical data," Mach Learn, vol. 20, no. 3, pp. 197-243, Sep. 1995.

Y. Fukushige, Fukushige: Representing probabilistic knowledge in... Google Scholar. ... the W3C Workshop on Semantic Web for Life Sciences, 2004.

A. DEVITT, B. Danev, and K. Matusikova, "Constructing bayesian networks automatically using ontologies," 2006.

S. Fenz and T. Neubauer, "How to determine threat probabilities using ontologies and Bayesian networks," presented at the CSIIRW '09:

Proceedings of the 5th Annual Workshop on Cyber Security and Information Intelligence Research: Cyber Security and Information Intelligence Challenges and Strategies, 2009.

[65] S. Fenz, A. M. Tjoa, and M. Hudec, "Ontology-Based Generation of Bayesian Networks," presented at the Complex, Intelligent and Software Intensive Systems, 2009. CISIS '09. International Conference on, 2009, pp. 
$712-717$.

[66] E. Foucar, "Classification in anatomic pathology," Am J Clin Pathol., vol. 116, no. Suppl, pp. S5-20., 2001.

[67] S. Swerdlow, C. International Agency for Research on, World Health, Organization, WHO classification of tumours of haematopoietic and lymphoid tissues. International Agency for Research on Cancer, 2008.

C. R. Taylor, "IHC and the WHO classification of lymphomas: cost effective immunohistochemistry using a deductive reasoning 'decision tree' approach," Appl Immunohistochem Mol Morphol, vol. 17, no. 5, pp. 366-374, Oct. 2009. C. R. Taylor, "The WHO classification of lymphomas: cost-effective immunohistochemistry using a deductive reasoning 'decision tree' approach: part II: the decision tree approach: diffuse patterns of proliferation in lymph nodes," Appl Immunohistochem Mol Morphol, vol. 17, no. 6, pp. 470-482, Dec. 2009.

[70] "Human Cell Differentiation Molecules," hcdm.org. [Online]. Available: http://www.hcdm.org/MoleculeInformation/tabid/54/Default.aspx. [Accessed: 24-Jun.-2012].

[71] T. Kishimoto, S. Goyert, H. Kikutani, D. Mason, M. Miyasaka, L. Moretta, T. Ohno, K. Okumura, S. Shaw, T. A. Springer, K. Sugamura, H. Sugawara, A. E. von dem Borne, and H. Zola, "CD antigens 1996," Blood, vol. 89, no. 10, p. 3502, May 1997.

[72] H. Zola and B. Swart, "The human leucocyte differentiation antigens (HLDA) workshops: the evolving role of antibodies in research, diagnosis and therapy," Cell Res, vol. 15, no. 9, pp. 691-694, Sep. 2005.

[73] H. Zola, B. Swart, A. Banham, S. Barry, A. Beare, A. Bensussan, L. Boumsell, D. B. C, H. J. Buhring, G. Clark, P. Engel, D. Fox, B. Q. Jin, P. J. Macardle, F. Malavasi, D. Mason, H. Stockinger, and X. Yang, "CD molecules 2006--human cell differentiation molecules," J Immunol Methods, vol. 319, no. 1, pp. 1-5, Jan. 2007.

[74] T. M. Elsheikh, S. L. Asa, J. K. Chan, R. A. DeLellis, C. S. Heffess, V. A. LiVolsi, and B. M. Wenig, "Interobserver and intraobserver variation among experts in the diagnosis of thyroid follicular lesions with borderline nuclear features of papillary carcinoma," Am J Clin Pathol., vol. 130, no. 5, pp. 736744, Nov. 2008.

[75] B. A. Friedman, "A survey of the myriad forces changing anatomic pathology and their consequences," Arch Pathol Lab Med, vol. 132, no. 5, pp. 735-738, May 2008.

[76] A. R. Jara-Lazaro, T. P. Thamboo, M. Teh, and P. H. Tan, "Digital pathology: exploring its applications in diagnostic surgical pathology practice," Pathology, vol. 42, no. 6, pp. 512-518, 2010.

[77] L. Pantanowitz, "Digital images and the future of digital pathology," Journal of pathology informatics, vol. 1, 2010.

[78] D. E. Heckerman, E. J. Horvitz, and B. N. Nathwani, "Toward normative expert systems: Part I. The Pathfinder project," Methods Inf Med, vol. 31, no. 2, pp. 90-105, Jun. 1992.

[79] R. T. Vollmer, "Differential diagnosis in immunohistochemistry with Bayes 
theorem," Am J Clin Pathol., vol. 131, no. 5, pp. 723-730, May 2009. R. T. Vollmer, "Primary lung cancer vs metastatic breast cancer: a probabilistic approach," Am J Clin Pathol., vol. 132, no. 3, pp. 391-395, Sep. 2009.

R. L. K. M. N. N. A. R. H. N. A. S. F. E. H. L. M. A. M. B. L. D. E. M. S. D. E. B. Horner MJ, Ed., "SEER Cancer Statistics Review," seer.cancer.gov, 2006. [Online]. Available: http://www.seer.cancer.gov/csr/1975_2006/. [Accessed: 24-Jun.-2012].

C. E. Shannon, "A mathematical theory of communication," Bell system technical journal, vol. 27, 1948.

M. H. Dunham, Data mining introductory and advanced topics. Upper Saddle River, N.J.: Prentice Hall/Pearson Education, 2003, pp. xiii-315 p. C. Shannon, A Mathematical Theory of Communication. CSLI Publications, 1948.

UniProt Consortium, "Reorganizing the protein space at the Universal Protein Resource (UniProt).," Nucleic Acids Res, vol. 40, no. Database issue, pp. D71-5, Jan. 2012.

J. W. 3. Huss, C. Orozco, J. Goodale, C. Wu, S. Batalov, T. J. Vickers, F. Valafar, and A. I. Su, "A gene wiki for community annotation of gene function," PLoS Biol, vol. 6, no. 7, p. e175, Jul. 2008.

E. Foucar, "Diagnostic decision-making in anatomic pathology," Am J Clin Pathol., vol. 116, pp. S21-33, Dec. 2001.

G. P. Pena and S. Andrade-Filho Jde, "How does a pathologist make a diagnosis?," Arch Pathol Lab Med, vol. 133, no. 1, pp. 124-132, Jan. 2009. G. Kapke and J. J. Stoddard, "Biomarkers - boon or bane for researchers?," Good Clinical Practice Journal, Oct. 2008.

M. L. Gatza, J. E. Lucas, W. T. Barry, J. W. Kim, Q. Wang, M. D. Crawford, M. B. Datto, M. Kelley, B. Mathey-Prevot, A. Potti, and J. R. Nevins, "A pathway-based classification of human breast cancer," Proc Natl Acad Sci U S A, vol. 107, no. 15, pp. 6994-6999, Apr. 2010.

K. Micklem and J. Sanderson, "Digital Imaging in Pathology," Current Diagnostic Pathology, no. 7, 2001.

Aperio, "ScanScope Digital Slide Scanners," aperio.com, 2008. [Online]. Available: http://aperio.com/pathology-services/scanscope-digitalscanners.asp. [Accessed: 24-Jun.-2012].

T. Verma and J. Pearl, "An algorithm for deciding if a set of observed independencies has a causal explanation," presented at the Eighth Conference on Uncertainty in Artificial Intelligence, 1992, p. 13. J. Pearl and T. S. Verma, "A theory of inferred causation," in Logic, methodology and philosophy of science, IX (Uppsala, 1991), vol. 134, Amsterdam: North-Holland, 1994, pp. 789-811.

J. Pearl, A. Paz, University of California, Los Angeles. Computer Science Department, Cognitive Systems Laboratory, GRAPHOIDS. 1986, p. 13. J. Pearl, Causality : models, reasoning, and inference. Cambridge, U.K. ; New York: Cambridge University Press, 2000, pp. xvi-384 p. "BioPAX Ontology Homepage," biopax.org. [Online]. Available: 
http://www.biopax.org/index.html. [Accessed: 17-Nov.-2011].

W3C, "Semantic Web," w3.org. [Online]. Available:

http://www.w3.org/standards/semanticweb/. [Accessed: 17-Nov.-2011].

[99] "Oracle 11g, Siebel, PeopleSoft | Oracle, The World's Largest Enterprise Software Company."

[100] K. Prasad, K. Kandasamy, and A. Pandey, "Human Protein Reference Database and Human Proteinpedia as discovery tools for systems biology," Reverse Chemical Genetics, vol. 577, pp. 67-79, 2009.

[101] C. Schaefer, K. Anthony, S. Krupa, J. Buchoff, M. Day, T. Hannay, and K. Buetow, "PID: the Pathway Interaction Database," Nucleic Acids Res, vol. 37, no. 1, pp. D674-D679, 2009.

[102] W. Barker, J. Garavelli, H. Huang, P. McGarvey, B. Orcutt, G. Srinivasarao, C. Xiao, L.-S. Yeh, R. Ledley, J. Janda, F. Pfeiffer, H.-W. Mewes, A.

Tsugita, and C. Wu, "The Protein Information Resource (PIR)," Nucl. Acids Res., vol. 28, no. 1, pp. 41-44, 2000.

[103] "BioCarta - Charting Pathways of Life," biocarta.com. [Online]. Available: http://www.biocarta.com/genes/index.asp. [Accessed: 17-Nov.-2011].

[104] S. A. Forbes, G. Bhamra, S. Bamford, E. Dawson, C. Kok, J. Clements, A. Menzies, J. W. Teague, P. A. Futreal, and M. R. Stratton, "The Catalogue of Somatic Mutations in Cancer (COSMIC)," Current protocols in human genetics / editorial board, Jonathan L. Haines ... [et al.], vol. 10, 2008.

[105] E. Prud'hommeaux and A. Seaborne, "SPARQL Query Language for RDF," 2008.

[106] K. Wilkinson, C. Sayers, and H. Kuno, "Efficient RDF storage and retrieval in Jena2," presented at the Proceedings of SWDB, 2003.

[107] R. Scheines, P. Spirtes, C. Glymour, C. Meek, and T. Richardson, "The TETRAD Project: Constraint Based Aids to Causal Model Specification," Multivariate Behav Res, vol. 33, no. 1, pp. 65-117, Jan. 1998.

[108] M. Hall, E. Frank, G. Holmes, B. Pfahringer, P. Reutemann, and I. H. Witten, "The WEKA data mining software: an update," SIGKDD Explorations Newsletter, vol. 11, no. 1, Nov. 2009.

[109] A. C. Gingras, B. Raught, and N. Sonenberg, "Regulation of translation initiation by FRAP/mTOR.," Genes Dev., vol. 15, no. 7, pp. 807-826, Apr. 2001.

[110] H. Khatib, W. Huang, D. Mikheil, V. Schutzkus, and R. L. Monson, "Effects of signal transducer and activator of transcription (STAT) genes STAT1 and STAT3 genotypic combinations on fertilization and embryonic survival rates in Holstein cattle.," J. Dairy Sci., vol. 92, no. 12, pp. 6186-6191, Dec. 2009.

[111] X. Zhang, P. Yue, S. Fletcher, W. Zhao, P. T. Gunning, and J. Turkson, "A novel small-molecule disrupts Stat3 SH2 domain-phosphotyrosine interactions and Stat3-dependent tumor processes.," Biochem. Pharmacol., vol. 79, no. 10, pp. 1398-1409, May 2010.

[112] S. Takemoto, K. Ushijima, K. Kawano, T. Yamaguchi, A. Terada, N. Fujiyoshi, S. Nishio, N. Tsuda, M. Ijichi, T. Kakuma, M. Kage, D. Hori, and T. Kamura, "Expression of activated signal transducer and activator of transcription-3 predicts poor prognosis in cervical squamous-cell carcinoma.," 
Br. J. Cancer, vol. 101, no. 6, pp. 967-972, Sep. 2009.

[113] R. Jiang, Z. Jin, Z. Liu, L. Sun, L. Wang, and K. Li, "Correlation of activated STAT3 expression with clinicopathologic features in lung adenocarcinoma and squamous cell carcinoma.," Molecular diagnosis \& therapy, vol. 15, no. 6, pp. 347-352, Dec. 2011.

[114] T. Morikawa, Y. Baba, M. Yamauchi, A. Kuchiba, K. Nosho, K. Shima, N. Tanaka, C. Huttenhower, D. A. Frank, C. S. Fuchs, and S. Ogino, "STAT3 expression, molecular features, inflammation patterns, and prognosis in a database of 724 colorectal cancers.," Clin. Cancer Res., vol. 17, no. 6, pp. 1452-1462, Mar. 2011.

[115] C. Huang, R. Huang, W. Chang, T. Jiang, K. Huang, J. Cao, X. Sun, and Z. Qiu, "The expression and clinical significance of pSTAT3, VEGF and VEGF-C in pancreatic adenocarcinoma.," Neoplasma, vol. 59, no. 1, pp. 5261, 2012.

[116] H.-S. Seo, H.-S. Choi, S.-R. Kim, Y. K. Choi, S.-M. Woo, I. Shin, J.-K. Woo, S.-Y. Park, Y. C. Shin, and S.-K. Ko, "Apigenin induces apoptosis via extrinsic pathway, inducing p53 and inhibiting STAT3 and NFאB signaling in HER2-overexpressing breast cancer cells.," Mol. Cell. Biochem., vol. 366, no. 1, pp. 319-334, Jul. 2012.

[117] S.-P. Teng, W.-L. Hsu, C.-Y. Chiu, M.-L. Wong, and S.-C. Chang, "Overexpression of P-glycoprotein, STAT3, phospho-STAT3 and KIT in spontaneous canine cutaneous mast cell tumours before and after prednisolone treatment.," Vet J, Mar. 2012.

[118] S. Umehara, H. Fujiwara, A. Shiozaki, M. Todo, A. Furutani, M. Yoneda, A. Ikai, H. Tada, S. Komatsu, D. Ichikawa, K. Okamoto, T. Ochiai, Y. Kokuba, and E. Otsuji, "PSK induces apoptosis through the inhibition of activated STAT3 in human esophageal carcinoma cells.," Int. J. Oncol., vol. 41, no. 1, pp. 61-66, Jul. 2012.

[119] E. Katz, A. H. Sims, D. Sproul, H. Caldwell, M. J. Dixon, R. R. Meehan, and D. J. Harrison, "Targeting of Rac GTPases blocks the spread of intact human breast cancer.," Oncotarget, Jun. 2012.

[120] D.-B. Liu, G.-Y. Hu, G.-X. Long, H. Qiu, Q. Mei, and G.-Q. Hu, “Celecoxib induces apoptosis and cell-cycle arrest in nasopharyngeal carcinoma cell lines via inhibition of STAT3 phosphorylation.," Acta Pharmacol. Sin., vol. 33, no. 5, pp. 682-690, May 2012.

[121] R. R. Nair, J. H. Tolentino, and L. A. Hazlehurst, "Role of STAT3 in Transformation and Drug Resistance in CML.," Front Oncol, vol. 2, p. 30, 2012.

[122] K. Swiatek-Machado, J. Mieczkowski, A. Ellert-Miklaszewska, P. Swierk, I. Fokt, S. Szymanski, S. Skora, W. Szeja, G. Grynkiewicz, B. Lesyng, W. Priebe, and B. Kaminska, "Novel small molecular inhibitors disrupt the JAK/STAT3 and FAK signaling pathways and exhibit a potent antitumor activity in glioma cells.," Cancer Biol. Ther., vol. 13, no. 8, pp. 657-670, Jun. 2012.

[123] X. Zhang, P. Yue, B. D. G. Page, T. Li, W. Zhao, A. T. Namanja, D. Paladino, J. Zhao, Y. Chen, P. T. Gunning, and J. Turkson, “Orally 
bioavailable small-molecule inhibitor of transcription factor Stat3 regresses human breast and lung cancer xenografts.," Proc Natl Acad Sci U S A, vol. 109, no. 24, pp. 9623-9628, Jun. 2012.

[124] A. Bonetto, T. Aydogdu, X. Jin, Z. Zhang, R. Zhan, L. Puzis, L. G. Koniaris, and T. A. Zimmers, "JAK/STAT3 pathway inhibition blocks skeletal muscle wasting downstream of IL-6 and in experimental cancer cachexia.," Am J Physiol Endocrinol Metab, Jun. 2012.

[125] G. Han, J. Y. Yu, Y. D. Chen, X. L. Cao, J. Zhu, W. Wang, X. X. Wang, X. Zhang, J. Q. Yan, and J. P. Gao, "The usefulness of phosphorylated-signal transduction and activators of transcription 3 in detecting prostate cancer from negative biopsies.," Eur J Surg Oncol, vol. 38, no. 4, pp. 367-373, Apr. 2012 .

[126] H. L. M. Koskela, S. Eldfors, P. Ellonen, A. J. van Adrichem, H. Kuusanmäki, E. I. Andersson, S. Lagström, M. J. Clemente, T. Olson, S. E. Jalkanen, M. M. Majumder, H. Almusa, H. Edgren, M. Lepistö, P. Mattila, K. Guinta, P. Koistinen, T. Kuittinen, K. Penttinen, A. Parsons, J. Knowles, J. Saarela, K. Wennerberg, O. Kallioniemi, K. Porkka, T. P. Loughran, C. A. Heckman, J. P. Maciejewski, and S. Mustjoki, "Somatic STAT3 mutations in large granular lymphocytic leukemia.," N. Engl. J. Med., vol. 366, no. 20, pp. 1905-1913, May 2012.

[127] H. Yang, A.-P. Xu, J. Lv, and Y. Liu, "[Expression of STAT3 and PIAS3 in renal tissues of MRL/lpr mice].," Nan Fang Yi Кe Da Хие Хие Bao, vol. 32, no. 6, pp. 821-825, Jun. 2012.

[128] J. Dean and S. Ghemawat, "MapReduce," Communications of the ACM, vol. 51, no. 1, pp. 107-113, Jan. 2008.

[129] V. Khadilkar, M. Kantarcioglu, and B. Thuraisingham, "Jena-HBase: A Distributed, Scalable and Efficient RDF Triple Store," utdallas.edu. 


\section{VITA}

Dmitriy Shin was born in Russia. After graduating from high school in 1986, he attended the Russian State Technological University of K.E. Tsiolkovsky in Moscow where he studied Computer Science. He then attended Moscow State University of Instrument Engineering and Computer Science where he received a M.S. in Computer Science. In 2003, Dmitriy Shin and his family immigrated to the USA where he began to work at the University of Missouri, Department of Pathology and Anatomical Sciences.

Dmitriy Shin's research interest includes pathology informatics, cancer informatics, machine learning and computational intelligence. He received his $\mathrm{PhD}$ in Informatics from the University of Missouri Informatics Institute in July 2012

Dmitriy Shin has a wife, Olga, and two sons, Alex and Max. In his spare time he enjoys fly-fishing and photography as well as travelling. 